SI-HEP-2011-05

\title{
Form Factors and Strong Couplings of Heavy Baryons from QCD Light-Cone Sum Rules
}

\author{
A. Khodjamirian, Ch. Klein, Th. Mannel, Y.-M. Wang \\ Theoretische Elementarteilchenphysik, Department Physik, \\ Universität Siegen, D-57068 Siegen, Germany
}

We derive QCD light-cone sum rules for the hadronic matrix elements of the heavy baryon transitions to nucleon. In the correlation functions the $\Lambda_{c}, \Sigma_{c}$ and $\Lambda_{b}$-baryons are interpolated by three-quark currents and the nucleon distribution amplitudes are used. To eliminate the contributions of negative parity heavy baryons, we combine the sum rules obtained from different kinematical structures. The results are then less sensitive to the choice of the interpolating current. We predict the $\Lambda_{b} \rightarrow p$ form factor and calculate the widths of the $\Lambda_{b} \rightarrow p \ell \nu_{l}$ and $\Lambda_{b} \rightarrow p \pi$ decays. Furthermore, we consider double dispersion relations for the same correlation functions and derive the light-cone sum rules for the $\Lambda_{c} N D^{(*)}$ and $\Sigma_{c} N D^{(*)}$ strong couplings. Their predicted values can be used in the models of charm production in $p \bar{p}$ collisions. 


\section{Introduction}

Our understanding of heavy flavour physics is incomplete without a deeper insight in the processes with heavy-flavoured baryons. The (electro)weak decays of $\Lambda_{b}, \Lambda_{c}$ and other $b$ - or $c$-baryons, such as $\Lambda_{b} \rightarrow p \ell \nu_{\ell}, \Lambda_{c} \rightarrow \Lambda \ell \nu_{\ell}, \Lambda_{b} \rightarrow \Lambda \ell \bar{\ell}$ and $\Lambda_{b} \rightarrow \Lambda \gamma$, can provide valuable information on the underlying quark-flavour operators, in particular, on their spin structure. Some exclusive decay channels of heavy baryons are already being investigated at Tevatron and LHC (see e.g., [1]). For a comprehensive analysis of these processes the heavy-to-light baryon form factors have to be calculated in QCD. These form factors are also used in the factorization estimates for nonleptonic decays e.g., for $\Lambda_{b} \rightarrow p \pi$.

Another topical problem, which stems from a different physical context, concerns the charmed baryon and meson strong couplings to the nucleon, for example, the $\Lambda_{c} D N$ or $\Lambda_{c} D^{*} N$ couplings. These strong interaction parameters can be used as normalization inputs in the models of charm production in the proton-antiproton collisions, such as the future experiment PANDA [2].

The lattice QCD studies do not yet access the heavy-to-light baryon form factors, whereas strong couplings in general remain a problem for the lattice simulations. The situation is more advanced for the non-lattice techniques. Among them, the method of QCD light-cone sum rules [3] (LCSR), well developed to calculate the heavy-to-light meson form factors [4], is flexible enough to predict also the baryonic matrix elements. One possibility to derive LCSR for the baryon form factors is to consider, in full analogy with the meson case, the vacuum $\rightarrow$ nucleon correlation function and express the result of the operator-product expansion (OPE) near the light-cone in terms of the nucleon distribution amplitudes (DA's). The latter have been worked out in [5, 6]. This approach was applied to the nucleon electromagnetic form factors [7, 8, 9, where the second nucleon in the correlation function was interpolated with the three-quark current e.g., with the Ioffe current [10]. In order to access heavy baryons, e.g., $\Lambda_{c}$ or $\Lambda_{b}$, one has to use a three-quark current with one heavy $c$ or $b$ quark. Matching the QCD calculation result for the correlation function to the hadronic dispersion relation in the variable of the heavy-baryon momentum squared, one obtains the LCSR for a heavy-to-light form factor. Furthermore, a sum rule for the (heavy baryon)-(heavy meson)-nucleon strong coupling can be obtained from the double dispersion relation for the same correlation function. Originally, this method was used to calculate the $D^{*} D \pi$ coupling in [11].

The aim of this paper is to calculate the heavy-to-light baryon form factors and strong couplings from the LCSR with the nucleon DA's. In the literature, one can find several applications of LCSR [12, 13, 14, 15, 16] or other QCD sum rule techniques [17, 18, 19, 20] to the heavy-baryon form factors and strong couplings. There is however an important problem in the sum rules for baryons which is absent in the case of mesons. Whatever three-quark current one uses to interpolate a given baryon, not only the ground state with the positive parity $\left(J^{P}=1 / 2^{+}\right)$, but also a heavier baryon resonance with the negative parity $\left(J^{P}=1 / 2^{-}\right)$couples to that current. As a result, e.g., in the hadronic dispersion relation for the isospin-zero charmed baryons, the ground state $\Lambda_{c}$ is accompanied by a $\simeq 300 \mathrm{MeV}$ heavier negative-parity resonance $\Lambda_{c}(2595)$ [21], which we hereafter denote 
as $\Lambda_{c}^{*}$. In the $b$-baryon spectrum, the $\Lambda_{b}^{*}$ resonance with $J^{P}=1 / 2^{-}$is expected to have a similar mass difference with respect to $\Lambda_{b}$. Note that $\Lambda_{c(b)}^{*}$ is a $P$-wave state in terms of quark model, and not a radial excitation of $\Lambda_{c(b)}$. Also the mass difference of heavy-light baryons with $J^{P}=1 / 2^{-}$and $J^{P}=1 / 2^{+}$is smaller, than in the case of light-quark baryons. Consequently, the influence of negative-parity states on the sum rules for heavy-light baryon matrix elements is expected to be more significant than in the case of the nucleon form factors. Hence, a usual quark-hadron duality ansatz for the hadronic spectral density in QCD sum rules - one lowest $\Lambda_{c(b)}$ resonance plus continuum approximated with OPE - is not accurate. Moreover, we expect that this simplified ansatz is one of the main reasons why there is a substantial dependence of the sum rule results on the choice of the interpolating heavy-baryon current in the correlation function.

Several approaches were suggested in the literature to isolate the negative-parity baryons in QCD sum rules. In [22], the heavy quark limit was employed, making use of the fact that the contributions of positive (negative) parity baryons in the two-point sum rules are proportional to $[1+\not x]([1-\not \chi])$, where $v$ is the 4 -velocity vector of the heavy baryon. Hence, one can introduce a parity projection matrix for the correlation function and construct two separate sum rules for the positive and negative parity baryons. However, such a procedure only works for infinitely heavy baryons, hence, at finite masses it cannot guarantee a clean separation of the negative parity states, especially in the case of charmed baryons. Another possibility suggested in [23] is to introduce the step function $\theta\left(x_{0}\right)$ in the correlation function and separate the contributions from baryons with different parities. One advantage of this ansatz is that it also works for light baryons.

In what follows, we adopt a new approach, including the contributions of negative parity baryons explicitly in the hadronic dispersion relations. The idea is very simple: we use a linear combination of the sum rules obtained from different kinematical structures of the same correlation function, so that the negative-parity baryon terms are cancelled out and only the ground-state baryon contribution is retained. The advantage of this procedure is that it does not rely on the heavy quark limit. Also, as we shall see from the numerical results, the form factor determination from LCSR becomes largely insensitive (within the uncertainties of our calculation) to the choice of the interpolating baryonic current.

The main phenomenological results obtained in this paper include the form factors of $\Lambda_{b} \rightarrow p$ transition and the strong couplings $\Lambda_{c} N D^{(*)}$ and $\Sigma_{c} N D^{(*)}$. The plan of the paper is as follows. In Sect. 2 we introduce the correlation functions and discuss the choice of the quark currents. In Sect. 3 we derive the hadronic dispersion relations for these correlation functions. In Sect. 4 the LCSR for the heavy-baryon $\rightarrow$ nucleon form factors are obtained, calculating the correlation functions in terms of the nucleon DA's and matching them to the dispersion relations. Since the form factors enter LCSR together with the decay constants of heavy baryons, in Sect. 5 we describe the two-point QCD sum rules used for these constants. In Sect. 6, the LCSR for the strong couplings are derived. The details of the numerical analysis of the form factors and strong couplings are collected in Sect. 7. Sect. 8 contains our predictions for the exclusive semileptonic 
$\Lambda_{b} \rightarrow p \ell \nu_{\ell}$ and nonleptonic $\Lambda_{b} \rightarrow p \pi$ decays based on the form factors obtained from LCSR. Sect. 9 is reserved for the concluding discussion. The paper contains several appendices where the bulky expressions of the nucleon DA's (App. A), LCSR for the form factors (Apps. B, C), the two-point sum rules for the decay constants (App. D) and the double spectral densities used in LCSR for the strong couplings (App. E) are collected.

\section{Correlation function and interpolating currents}

As a first step to derive the LCSR, we introduce the following vacuum-to-nucleon correlation function:

$$
\Pi_{a}(P, q)=i \int d^{4} z e^{i q \cdot z}\left\langle 0\left|T\left\{\eta(0), j_{a}(z)\right\}\right| N(P)\right\rangle .
$$

In the above, the current $\eta$ interpolating a heavy-light baryon and the current $j_{a}$ of the heavy-light transition ( $a$ indicates a certain Lorentz structure) enter the $T$-product, sandwiched between the nucleon on-shell state $|N\rangle$ with the four-momentum $P\left(P^{2}=m_{N}^{2}\right)$ and the vacuum. The heavy-quark mass $m_{Q}$ is finite, and the calculation is applicable to both charmed $\left(m_{Q}=m_{c}\right)$ and beauty $\left(m_{Q}=m_{b}\right)$ baryons. Moreover, a generalization to the case of strange baryons is possible in the same framework $\left(m_{Q} \rightarrow m_{s}\right)$, provided the external momentum transfer $q$ is deep spacelike. For the heavy quarks this condition is fulfilled if $q^{2} \ll m_{Q}^{2}$. Note that the $N$-state is taken as an initial one in (11) for simplicity, in order to directly use the definitions of the nucleon DA's from [5]. In the phenomenological applications of our interest $N$ is a proton.

For definiteness, in what follows we consider the correlation function (11) with the $c$ quark, selecting the flavour configuration $u d c$ for the baryon interpolating current and, correspondingly, $\bar{c} u$ for the transition current. With this choice, we first derive LCSR for the $\Lambda_{c} \rightarrow p$ and $\Sigma_{c} \rightarrow p$ form factors. Switching from $c$ to $b$ quark in the $\Lambda_{c} \rightarrow p$ sum rules (and accordingly adjusting the relevant scales in the correlation function), we obtain the LCSR for $\Lambda_{b} \rightarrow p$ form factors, our first phenomenological goal. Furthermore, the flavour configuration chosen in (1) leads, via double dispersion relations, to LCSR for the strong couplings of charmed baryons $\Lambda_{c}, \Sigma_{c}$ with the nucleon and $D^{(*)}$-mesons.

In what follows, we consider the heavy-light transition currents with pseudoscalar, vector and axial-vector quantum numbers:

$$
j_{a}=\bar{c} \Gamma_{a} u, \text { with } \Gamma_{a}=m_{c} i \gamma_{5}, \gamma_{\mu}, \gamma_{\mu} \gamma_{5},
$$

respectively. For the sake of renormalization invariance, the quark mass is inserted in the pseudoscalar current.

For the heavy-light baryon interpolating current we have the following general structure:

$$
\eta=\epsilon^{i j k}\left(u_{i} C \Gamma_{b} d_{j}\right) \widetilde{\Gamma}_{b} c_{k},
$$

where the first fermion field $u_{i}$ should be hereafter understood as $u_{i}^{\mathrm{T}}, C$ is the charge conjugation matrix, and the sum goes over the colour indices $i, j, k$. There are multiple 
choices for the Dirac structures $\Gamma_{b}$ and $\widetilde{\Gamma}_{b}$ in the above current. The discussion of the optimal choice of the baryon interpolating current goes back to the early papers [10, 24, 25]. In the $\Lambda_{c}$-baryon, the isospin of the light diquark [ud] is zero, excluding the structures $\gamma_{\mu}$ and $\sigma_{\mu \nu}$ for $\Gamma_{b}$ in the $\eta$ current. Still there is a freedom to choose in (3) the following combinations: $\Gamma_{b}=\gamma_{5}, \widetilde{\Gamma}_{b}=\mathbb{1}$, leading to

$$
\eta=\eta_{\Lambda_{c}}^{(\mathcal{P})}=\left(u C \gamma_{5} d\right) c
$$

or $\Gamma_{b}=\gamma_{5} \gamma_{\lambda}, \quad \widetilde{\Gamma}_{b}=\gamma^{\lambda}$, for which

$$
\eta=\eta_{\Lambda_{c}}^{(\mathcal{A})}=\left(u C \gamma_{5} \gamma_{\lambda} d\right) \gamma^{\lambda} c
$$

In addition, a simpler current

$$
\eta_{\Lambda_{c}}^{(\mathcal{S})}=(u C d) \gamma_{5} c
$$

is also possible, as well as any linear combination of all three above currents.

The heavy-quark limit [24] provides another guiding principle for choosing an optimal heavy baryon current, at least at the qualitative level. In particular, since the light diquark system in the current (6) is in the $P$-wave, this current is not expected [22] to have a considerable overlap with the ground-state $\Lambda_{c}$. Hence, in what follows we will only leave (41) and (5) under consideration, which we denote as the pseudoscalar and axial-vector currents, respectively. The correlation functions (1) with the pseudoscalar (axial-vector) interpolating current and with the transition currents listed in (2) are denoted as $\Pi_{5}^{(\mathcal{P})}, \Pi_{\mu}^{(\mathcal{P})}, \Pi_{\mu 5}^{(\mathcal{P})}\left(\Pi_{5}^{(\mathcal{A})}, \Pi_{\mu}^{(\mathcal{A})}, \Pi_{\mu 5}^{(\mathcal{A})}\right)$, respectively.

Turning to the $\Sigma_{c}$ baryon where the light diquark $[u d]$ has isospin one, we again adopt two different currents: the Ioffe current [10] with $\Gamma_{b}=\gamma_{\lambda}$, and $\widetilde{\Gamma}_{b}=\gamma^{\lambda} \gamma_{5}$ :

$$
\eta_{\Sigma_{c}}^{(\mathcal{I})}=\left(u C \gamma_{\lambda} d\right) \gamma^{\lambda} \gamma_{5} c
$$

and the tensor current with $\Gamma_{b}=\sigma_{\mu \nu}$ and $\widetilde{\Gamma}_{b}=\sigma^{\mu \nu} \gamma_{5}$ :

$$
\eta_{\Sigma_{c}}^{(\mathcal{T})}=\left(u \sigma_{\mu \nu} d\right) \sigma^{\mu \nu} \gamma_{5} c
$$

The Ioffe current is used in the LCSR for the nucleon form factors [8]. One advantage of this current is that the power corrections terms are small. On the other hand, the tensor current provides a reduced continuum contributions, at least in the sum rules with light baryons [25].

\section{Accessing the form factors with hadronic dispersion relations}

We begin with the hadronic transitions involving $\Lambda_{c}$. Following the usual procedure of the QCD sum rule derivation, we insert in the correlation function (11) a total set of charmed-baryon states between the interpolating current $\eta=\eta_{\Lambda_{c}}^{(i)}($ where $i=\mathcal{P}$ or $\mathcal{A}$ ) and the transition current $j_{a}$. In the resulting hadronic dispersion relation the contributions 
of the lowest state $\Lambda_{c}$ and its negative-parity partner $\Lambda_{c}^{*}$ enter. The residue of the $\Lambda_{c^{-}}$ pole contains the product of two hadronic matrix elements. The first one is the coupling of $\Lambda_{c}$ with the interpolating current $\eta_{\Lambda_{c}}^{(i)}$ (the decay constant), defined as

$$
\left\langle 0\left|\eta_{\Lambda_{c}}^{(i)}\right| \Lambda_{c}(P-q)\right\rangle=m_{\Lambda_{c}} \lambda_{\Lambda_{c}}^{(i)} u_{\Lambda_{c}}(P-q),
$$

where $u_{\Lambda_{c}}(P-q)$ is the $\Lambda_{c}$-bispinor with the four-momentum $(P-q)$. The second hadronic matrix element $\left\langle\Lambda_{c}(P-q)\left|j_{a}\right| N(P)\right\rangle$ is defined in terms of $N \rightarrow \Lambda_{c}$ form factors which are equal to the $\Lambda_{c} \rightarrow N$ form factors of our interest, up to an inessential general phase.

For the pseudoscalar transition current there is only one form factor, which we define as:

$$
\left\langle\Lambda_{c}(P-q)\left|m_{c} \bar{c} i \gamma_{5} u\right| N(P)\right\rangle=\left(m_{\Lambda_{c}}+m_{N}\right) G\left(q^{2}\right) \bar{u}_{\Lambda_{c}}(P-q) i \gamma_{5} u_{N}(P),
$$

where the mass-dependent factor on r.h.s. is introduced to keep the form factor $G\left(q^{2}\right)$ dimensionless. The hadronic matrix elements with the vector and axial-vector transition currents contain three form factors each:

$$
\begin{aligned}
& \left\langle\Lambda_{c}(P-q)\left|\bar{c} \gamma_{\mu} u\right| N(P)\right\rangle=\bar{u}_{\Lambda_{c}}(P-q)\left\{f_{1}\left(q^{2}\right) \gamma_{\mu}+i \frac{f_{2}\left(q^{2}\right)}{m_{\Lambda_{c}}} \sigma_{\mu \nu} q^{\nu}+\frac{f_{3}\left(q^{2}\right)}{m_{\Lambda_{c}}} q_{\mu}\right\} u_{N}(P), \\
& \left\langle\Lambda_{c}(P-q)\left|\bar{c} \gamma_{\mu} \gamma_{5} u\right| N(P)\right\rangle=\bar{u}_{\Lambda_{c}}(P-q)\left\{g_{1}\left(q^{2}\right) \gamma_{\mu}+i \frac{g_{2}\left(q^{2}\right)}{m_{\Lambda_{c}}} \sigma_{\mu \nu} q^{\nu}+\frac{g_{3}\left(q^{2}\right)}{m_{\Lambda_{c}}} q_{\mu}\right\} \gamma_{5} u_{N}(P) .
\end{aligned}
$$

Taking the divergence of the axial-vector current one obtains the following relation:

$$
G\left(q^{2}\right)=g_{1}\left(q^{2}\right)-\frac{q^{2}}{m_{\Lambda_{c}}\left(m_{\Lambda_{c}}+m_{N}\right)} g_{3}\left(q^{2}\right) .
$$

The $\Lambda_{c}^{*}$-pole term in the dispersion relation contains the decay constant

$$
\left\langle 0\left|\eta_{\Lambda_{c}}^{(i)}\right| \Lambda_{c}^{*}(P-q)\right\rangle=m_{\Lambda_{c}^{*}} \lambda_{\Lambda_{c}^{*}}^{(i)} \gamma_{5} u_{\Lambda_{c}^{*}}(P-q),
$$

which is multiplied with the form factor of the $N \rightarrow \Lambda_{c}^{*}$ transition. For the pseudoscalar transition current we define this form factor as

$$
\left\langle\Lambda_{c}^{*}(P-q)\left|m_{c} \bar{c} i \gamma_{5} u\right| N(P)\right\rangle=\left(m_{\Lambda_{c}^{*}}-m_{N}\right) \widetilde{G}\left(q^{2}\right) i \bar{u}_{\Lambda_{c}^{*}}(P-q) u_{N}(P) .
$$

For the vector and axial-vector currents the definitions of the corresponding form factors $\tilde{f}_{1,2,3}\left(q^{2}\right)$ and $\tilde{g}_{1,2,3}\left(q^{2}\right)$ are obtained from (11) and (12), respectively, adding $\gamma_{5}$ after the $\Lambda_{c}$ bispinor and replacing $\Lambda_{c} \rightarrow \Lambda_{c}^{*}$.

Taking into account the equation of motion $\left(\not P-m_{N}\right) u_{N}(P)=0$, we decompose the correlation function (11) in independent invariant amplitudes. In the case of the pseudoscalar transition current $j_{5}$ there are two amplitudes:

$$
\Pi_{5}^{(i)}(P, q)=\left[\Pi_{1}^{(i)}\left((P-q)^{2}, q^{2}\right)+q \Pi_{2}^{(i)}\left((P-q)^{2}, q^{2}\right)\right] i \gamma_{5} u_{N}(P),
$$


for both $i=\mathcal{P}$ or $\mathcal{A}$. In the case of the vector current $j_{\mu}$ there are six invariant amplitudes:

$$
\Pi_{\mu}^{(i)}(P, q)=\left(\widetilde{\Pi}_{1}^{(i)} P_{\mu}+\widetilde{\Pi}_{2}^{(i)} P_{\mu} q+\widetilde{\Pi}_{3}^{(i)} \gamma_{\mu}+\widetilde{\Pi}_{4}^{(i)} \gamma_{\mu} \phi+\widetilde{\Pi}_{5}^{(i)} q_{\mu}+\widetilde{\Pi}_{6}^{(i)} q_{\mu} q\right) u_{N}(P)
$$

where the dependence of $\widetilde{\Pi}_{1-6}^{(i)}$ on $(P-q)^{2}$ and $q^{2}$ is not shown for brevity; a similar decomposition for the correlation function $\Pi_{\mu 5}^{(i)}$ with the axial-vector current $j_{\mu 5}$ reads:

$\Pi_{\mu 5}^{(i)}(P, q)=\left(\bar{\Pi}_{1}^{(i)} P_{\mu}+\bar{\Pi}_{2}^{(i)} P_{\mu} \phi q+\bar{\Pi}_{3}^{(i)} \gamma_{\mu}+\bar{\Pi}_{4}^{(i)} \gamma_{\mu} \phi q+\bar{\Pi}_{5}^{(i)} q_{\mu}+\bar{\Pi}_{6}^{(i)} q_{\mu} \not\right) \gamma_{5} u_{N}(P)$.

Employing the above definitions of decay constants and form factors, and summing over the helicities of the $\Lambda_{c}$ and $\Lambda_{c}^{*}$, we obtain the hadronic dispersion relations for each invariant amplitude in (16), (17) and (18). In the case of the pseudoscalar current one has:

$$
\begin{array}{r}
\Pi_{1}^{(i)}\left((P-q)^{2}, q^{2}\right)=\frac{m_{\Lambda_{c}}\left(m_{\Lambda_{c}}^{2}-m_{N}^{2}\right) \lambda_{\Lambda_{c}}^{(i)} G\left(q^{2}\right)}{m_{\Lambda_{c}}^{2}-(P-q)^{2}} \\
+\frac{m_{\Lambda_{c}^{*}}\left(m_{\Lambda_{c}^{*}}^{2}-m_{N}^{2}\right) \lambda_{\Lambda_{c}^{*}}^{(i)} \tilde{G}\left(q^{2}\right)}{m_{\Lambda_{c}^{*}}^{2}-(P-q)^{2}}+\int_{s_{0}^{h}}^{\infty} d s \frac{\rho_{1}^{(i)}\left(s, q^{2}\right)}{s-(P-q)^{2}},
\end{array}
$$

and

$$
\begin{gathered}
\Pi_{2}^{(i)}\left((P-q)^{2}, q^{2}\right)=-\frac{m_{\Lambda_{c}}\left(m_{\Lambda_{c}}+m_{N}\right) \lambda_{\Lambda_{c}}^{(i)} G\left(q^{2}\right)}{m_{\Lambda_{c}}^{2}-(P-q)^{2}} \\
+\frac{m_{\Lambda_{c}^{*}}\left(m_{\Lambda_{c}^{*}}-m_{N}\right) \lambda_{\Lambda_{c}^{*}}^{(i)} \tilde{G}\left(q^{2}\right)}{m_{\Lambda_{c}^{*}}^{2}-(P-q)^{2}}+\int_{s_{0}^{h}}^{\infty} d s \frac{\rho_{2}^{(i)}\left(s, q^{2}\right)}{s-(P-q)^{2}},
\end{gathered}
$$

where the hadronic spectral densities of all excited and continuum states with the quantum numbers of $\Lambda_{c}$ and $\Lambda_{c}^{*}$ are denoted as $\rho_{1,2}^{(i)}$, and $s_{0}^{h}$ is the corresponding threshold. Possible subtractions are neglected, having in mind the subsequent Borel transformation.

In the case of the vector transition current the dispersion relations for the six independent invariant amplitudes have the same structure as (19) and (20). Instead of writing them down one by one, we present one combined expression for the correlation function, written in terms of the hadronic contributions:

$$
\begin{array}{r}
\Pi_{\mu}^{(i)}(P, q)=\frac{\lambda_{\Lambda_{c}}^{(i)} m_{\Lambda_{c}}}{m_{\Lambda_{c}}^{2}-(P-q)^{2}}\left[2 f_{1}\left(q^{2}\right) P_{\mu}-2 \frac{f_{2}\left(q^{2}\right)}{m_{\Lambda_{c}}} P_{\mu} \not q\right. \\
+\left(m_{\Lambda_{c}}-m_{N}\right)\left(f_{1}\left(q^{2}\right)-\frac{m_{\Lambda_{c}}+m_{N}}{m_{\Lambda_{c}}} f_{2}\left(q^{2}\right)\right) \gamma_{\mu}+\left(f_{1}\left(q^{2}\right)-\frac{m_{\Lambda_{c}}+m_{N}}{m_{\Lambda_{c}}} f_{2}\left(q^{2}\right)\right) \gamma_{\mu} \not q \\
\left.+\left(-2 f_{1}\left(q^{2}\right)+\frac{m_{\Lambda_{c}}+m_{N}}{m_{\Lambda_{c}}}\left(f_{2}\left(q^{2}\right)+f_{3}\left(q^{2}\right)\right)\right) q_{\mu}+\frac{1}{m_{\Lambda_{c}}}\left(f_{2}\left(q^{2}\right)-f_{3}\left(q^{2}\right)\right) q_{\mu} \not q\right] u_{N}(P)
\end{array}
$$




$$
\begin{array}{r}
+\frac{\lambda_{\Lambda_{c}^{*}}^{(i)} m_{\Lambda_{c}^{*}}}{m_{\Lambda_{c}^{*}}^{2}-(P-q)^{2}}\left[-2 \tilde{f}_{1}\left(q^{2}\right) P_{\mu}+2 \frac{\tilde{f}_{2}\left(q^{2}\right)}{m_{\Lambda_{c}^{*}}} P_{\mu} \not q\right. \\
+\left(m_{\Lambda_{c}^{*}}+m_{N}\right)\left(\tilde{f}_{1}\left(q^{2}\right)+\frac{m_{\Lambda_{c}^{*}}-m_{N}}{m_{\Lambda_{c}^{*}}} \tilde{f}_{2}\left(q^{2}\right)\right) \gamma_{\mu}-\left(\tilde{f}_{1}\left(q^{2}\right)+\frac{m_{\Lambda_{c}^{*}}-m_{N}}{m_{\Lambda_{c}}} \tilde{f}_{2}\left(q^{2}\right)\right) \gamma_{\mu} \not q \\
\left.+\left(2 \tilde{f}_{1}\left(q^{2}\right)+\frac{m_{\Lambda_{c}^{*}}-m_{N}}{m_{\Lambda_{c}^{*}}}\left(\tilde{f}_{2}\left(q^{2}\right)+\tilde{f}_{3}\left(q^{2}\right)\right)\right) q_{\mu}-\frac{1}{m_{\Lambda_{c}^{*}}}\left(\tilde{f}_{2}\left(q^{2}\right)-\tilde{f}_{3}\left(q^{2}\right)\right) q_{\mu} \not q\right] u_{N}(P) \\
+\int_{s_{0}^{h}}^{\infty} \frac{d s}{s-(P-q)^{2}}\left(\bar{\rho}_{1}^{(i)}\left(s, q^{2}\right) P_{\mu}+\tilde{\rho}_{2}^{(i)} P_{\mu} q\right. \\
\left.+\tilde{\rho}_{3}^{(i)} \gamma_{\mu}+\tilde{\rho}_{4}^{(i)} \gamma_{\mu} \phi+\tilde{\rho}_{5}^{(i)} q_{\mu}+\tilde{\rho}_{6}^{(i)} q_{\mu} \phi\right) u_{N}(P) .
\end{array}
$$

Collecting the coefficients at each bispinor structure in the above, we equate their sum to the amplitude $\widetilde{\Pi}_{1, \ldots, 6}^{(i)}$ which multiplies the same structure in the decomposition (17). The analogous hadronic decomposition for the correlation function $\Pi_{\mu 5}^{(i)}(P, q)$ with the axial-vector current can be obtained from (21) by replacing $f_{i} \rightarrow g_{i}, \tilde{f}_{i} \rightarrow \tilde{g}_{i}$, changing the sign of $m_{N}$ and adding $\gamma_{5}$ before the nucleon spinor.

The hadronic dispersion relations for the correlation function with the $\Sigma_{c}$ interpolating currents are obtained from the relations for the $\Lambda_{c}$ presented above by simple replacements $\Lambda_{c} \rightarrow \Sigma_{c}$ and $\Lambda_{c}^{*} \rightarrow \Sigma_{c}^{*}$. We identify the $\Sigma_{c}^{*}\left(1 / 2^{-}\right)$state with the resonance $\Sigma_{c}(2800)$ [21] whose mass is close to the expected one:

$$
m_{\Sigma_{c}^{*}} \simeq m_{\Sigma_{c}}+\left(m_{\Lambda_{c}^{*}}-m_{\Lambda_{c}}\right) \simeq 2764 \mathrm{MeV} .
$$

The dispersion relations obtained above will be used in the following section to derive the LCSR.

\section{Light-Cone Sum Rules for the form factors}

We now turn to the computation of the correlation function (11) for the $\Lambda_{c} \rightarrow p$ transition, employing two different interpolating currents for $\Lambda_{c}$ and, in each case, the three transition currents listed in (2). Throughout this calculation we neglect the light-quark masses everywhere; the only two mass parameters in the correlation function are the $c$-quark mass $m_{c}$ and the nucleon mass $m_{N}$, the latter entering the nucleon DA's. The external 4-momenta $P-q$ and $q$ are taken spacelike, $(P-q)^{2}, q^{2} \ll m_{c}^{2}$, to justify the expansion of the product of the two currents in (1I) near the light-cone $\left(z^{2} \sim 0\right)$. The OPE result is obtained as a sum over nucleon DA's of growing twist, convoluted with the hard-scattering amplitudes formed by the virtual $c$-quark propagator, as shown in the diagram of Fig. 1. We include all three-particle nucleon DA's from twist 3 to twist 6 . The contributions of soft gluons emitted from the $c$-quark and absorbed by the nucleon, demand the knowledge of the four-particle (three-quark-gluon) nucleon DA's. Their analysis has just started [26, 27]. In fact, the soft-gluon contributions to OPE are 


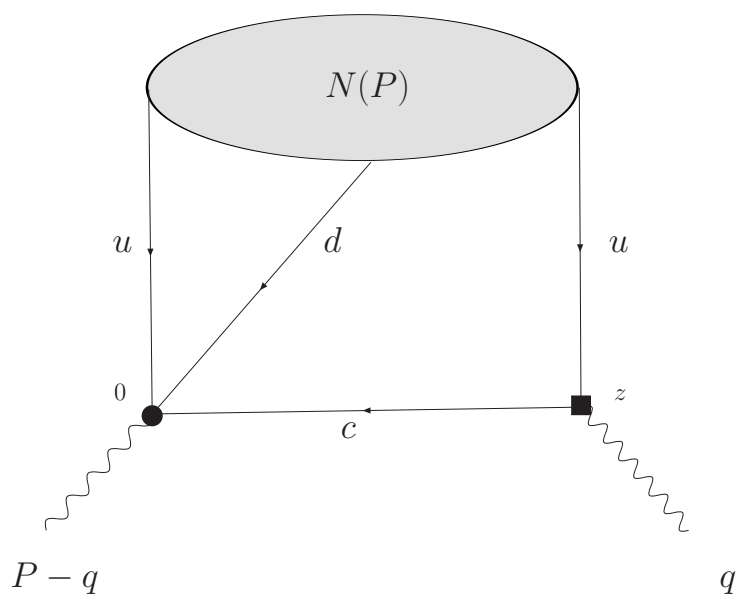

Figure 1: Diagrammatic representation of the correlation function (1). The wavy lines (oval) represent the external currents (the nucleon DA).

expected to be suppressed by extra powers of the virtual $c$-quark propagator. Another future improvement of LCSR is possible, if one calculates the $O\left(\alpha_{s}\right)$ corrections to the correlation function corresponding to the hard gluon exchanges between the quark lines in the diagram of Fig. 1.

The nucleon DA's at $z^{2} \rightarrow 0$ are defined according to [5]:

$$
\begin{array}{r}
\left\langle 0\left|\epsilon^{i j k} u_{\alpha}^{i}(0) u_{\beta}^{j}(z) d_{\gamma}^{k}(0)\right| N(P)\right\rangle=\mathcal{V}_{1}(P C)_{\alpha \beta}\left(\gamma_{5} u_{N}\right)_{\gamma}+\mathcal{A}_{1}\left(P \gamma_{5} C\right)_{\alpha \beta}\left(u_{N}\right)_{\gamma} \\
+\mathcal{T}_{1}\left(P^{\nu} i \sigma_{\mu \nu} C\right)_{\alpha \beta}\left(\gamma^{\mu} \gamma_{5} u_{N}\right)_{\gamma}+\ldots
\end{array}
$$

where $\alpha, \beta, \gamma$ are Dirac indices. The terms shown above receive their contributions from the lowest twist-3 DA's. The complete, rather bulky decomposition is presented in App. A. In (23), the calligraphic notations $\mathcal{F}=\left\{\mathcal{V}_{1}, \mathcal{A}_{1}, \mathcal{T}_{1}\right\}$ denote the integrals over the twist-3 nucleon DA's:

$$
\mathcal{F}=\int d x_{1} d x_{2} d x_{3} \delta\left(1-x_{1}-x_{2}-x_{3}\right) e^{-i x_{2} P \cdot z} F\left(x_{i}, \mu\right),
$$

denoted by the same noncalligraphic letters $F=\left\{V_{1}, A_{1}, T_{1}\right\}$, where $x_{i}=\left\{x_{1}, x_{2}, x_{3}\right\}$, $\left(0 \leq x_{i} \leq 1\right)$ are the longitudinal momentum fractions of the quarks in the nucleon and $\mu$ is the normalization scale. The twist-3 DA's:

$$
\begin{aligned}
V_{1}\left(x_{i}, \mu\right) & =120 x_{1} x_{2} x_{3}\left[\phi_{3}^{0}(\mu)+\phi_{3}^{+}(\mu)\left(1-3 x_{3}\right)\right], \\
A_{1}\left(x_{i}, \mu\right) & =120 x_{1} x_{2} x_{3}\left(x_{2}-x_{1}\right) \phi_{3}^{-}(\mu), \\
T_{1}\left(x_{i}, \mu\right) & =120 x_{1} x_{2} x_{3}\left[\phi_{3}^{0}(\mu)-\frac{1}{2}\left(\phi_{3}^{+}(\mu)-\phi_{3}^{-}(\mu)\right)\left(1-3 x_{3}\right)\right] .
\end{aligned}
$$

are derived in [5] where one can find the details. The expressions for the nucleon DA's of twist 4, 5, 6 as well as the relations for their normalization and shape parameters, such as $\phi_{3}^{0, \pm}$ in (25), are presented in App. A. 
Substituting the decomposition (23) in the correlation functions $\Pi_{5, \mu, \mu 5}^{(i)}$, we isolate the invariant amplitudes. The integration over the variable $x_{1}$ is performed easily, while the virtual $c$-quark momentum in the chosen configuration is equal to $\left(x_{2} P-q\right)$ and contains no $x_{1}$-dependence. The result is represented as a sum of integrals over the remaining variable $x_{2} \equiv x$.

For the correlation function with the pseudoscalar transition current, the invariant amplitudes defined in (16) can be transformed to the following form:

$$
\Pi_{j}^{(i)}\left((P-q)^{2}, q^{2}\right)=\frac{m_{c}}{4} \sum_{n=1,2,3} \int_{0}^{1} d x \frac{\omega_{j n}^{(i)}\left(x,(P-q)^{2}, q^{2}\right)}{D^{n}}
$$

with the denominator

$$
D=m_{c}^{2}-(x P-q)^{2}=m_{c}^{2}-x(P-q)^{2}-\bar{x} q^{2}+x \bar{x} m_{N}^{2},
$$

and $\bar{x}=1-x$. The functions $\omega_{j n}^{(i)}$ are distinguished by their indices: $i=\mathcal{P}, \mathcal{A}$ (baryon current), $j=1,2$ (the number of the invariant amplitude) and $n=1,2,3$ (the power of the denominator). They depend linearly on $(P-q)^{2}, q^{2}$ and polynomially or logarithmically on $x$. Note that in (26) we do not show the terms which vanish after Borel transformation in $(P-q)^{2}$. Since the invariant amplitudes in the form (26) will now be used in the LCSR for the form factors, we also replace $(P-q)^{2}$ in the numerators $\omega_{j n}^{(i)}\left(x,(P-q)^{2}, q^{2}\right)$ by $s(x)-D / x$, where

$$
s(x)=\left(m_{c}^{2}-\bar{x} q^{2}+x \bar{x} m_{N}^{2}\right) / x .
$$

The transformed functions $\omega_{j n}^{(i)}\left(x, q^{2}\right)$ are presented in App. B. For the correlation functions with the vector and axial-vector transition currents, expressions similar to (26) (without the factor $\left.m_{c}\right)$ are obtained for the invariant amplitudes $\widetilde{\Pi}_{j}^{(i)}$ and $\bar{\Pi}_{j}^{(i)}(j=$ $1, \ldots, 6)$. The corresponding numerator functions $\widetilde{\omega}_{j n}^{(i)}$ and $\bar{\omega}_{j n}^{(i)}$, respectively, are also given in App. B. Furthermore, App. C contains the numerator functions $\omega_{j n}^{(i)}, \widetilde{\omega}_{j n}^{(i)}$ and $\bar{\omega}_{j n}^{(i)}(i=\mathcal{I}, \mathcal{T})$ for the correlation functions with the $\Sigma_{c}$ interpolating currents.

After computing the OPE expressions for all invariant amplitudes in the integral form (26)), we use the hadronic dispersion relations for these amplitudes presented in the previous section. At this point, we notice that each form factor enters more than one dispersion relation. E.g., in the case of pseudoscalar current, there are two linearly independent relations (19) and (20) for $\Pi_{1}^{(i)}$ and $\Pi_{2}^{(i)}$, respectively, both containing the $\Lambda_{c^{-}}$and $\Lambda_{c}^{*}$-pole terms. Combining them, we eliminate the $\Lambda_{c}^{*}$ contributions, obtaining a linear combination of dispersion relations containing only the hadronic matrix elements for the ground-state $\Lambda_{c}$-baryon:

$$
\begin{array}{r}
\frac{m_{\Lambda_{c}}\left(m_{\Lambda_{c}}+m_{N}\right)\left(m_{\Lambda_{c}}+m_{\Lambda_{c}^{*}}\right) \lambda_{\Lambda_{c}}^{(i)} G\left(q^{2}\right)}{m_{\Lambda_{c}}^{2}-(P-q)^{2}}+\int_{s_{0}^{h}}^{\infty} d s \frac{\rho_{1}^{(i)}\left(s, q^{2}\right)-\left(m_{\Lambda_{c}^{*}}+m_{N}\right) \rho_{2}^{(i)}\left(s, q^{2}\right)}{s-(P-q)^{2}} \\
=\left[\Pi_{1}^{(i)}\left((P-q)^{2}, q^{2}\right)-\left(m_{\Lambda_{c}^{*}}+m_{N}\right) \Pi_{2}^{(i)}\left((P-q)^{2}, q^{2}\right)\right] .
\end{array}
$$


The contributions of the hadronic states above the threshold $s_{0}^{h}$ are approximated using quark-hadron duality:

$$
\begin{array}{r}
\int_{s_{0}^{h}}^{\infty} \frac{d s}{s-(P-q)^{2}}\left[\rho_{1}^{(i)}\left(s, q^{2}\right)-\left(m_{\Lambda_{c}^{*}}+m_{N}\right) \rho_{2}^{(i)}\left(s, q^{2}\right)\right] \\
=\frac{1}{\pi} \int_{s_{0}}^{\infty} \frac{d s}{s-(P-q)^{2}}\left[\operatorname{Im}_{s} \Pi_{1}^{(i)}\left(s, q^{2}\right)-\left(m_{\Lambda_{c}^{*}}+m_{N}\right) \operatorname{Im}_{s} \Pi_{2}^{(i)}\left(s, q^{2}\right)\right],
\end{array}
$$

where $s_{0}$ is the effective threshold parameter. The spectral densities $\operatorname{Im}_{s} \Pi_{1,2}^{(i)}$ are calculated from the OPE result (26). To this end, the integrals in (26) are transformed to the dispersion form in $(P-q)^{2}$, transforming the integration variable $x$ to $s(x)$ defined in (28), so that

$$
x(s)=\frac{1}{2 m_{N}^{2}}\left[m_{N}^{2}+q^{2}-s+\sqrt{\left(s-q^{2}-m_{N}^{2}\right)^{2}+4 m_{N}^{2}\left(m_{c}^{2}-q^{2}\right)}\right] .
$$

The final step in obtaining LCSR is the Borel transformation $(P-q)^{2} \rightarrow M^{2}$, introducing the Borel parameter $M^{2}$ in the charmed baryon channel. The resulting sum rule for the form factor reads:

$$
\begin{aligned}
& G\left(q^{2}\right)=\frac{e^{m_{\Lambda_{c}}^{2} / M^{2}}}{m_{\Lambda_{c}}\left(m_{\Lambda_{c}}+m_{N}\right)\left(m_{\Lambda_{c}}+m_{\Lambda_{c}^{*}}\right) \lambda_{\Lambda_{c}}^{(i)}} \frac{1}{\pi} \int_{m_{c}^{2}}^{s_{0}} d s e^{-s / M^{2}}\left[\operatorname{Im}_{s} \Pi_{1}^{(i)}\left(s, q^{2}\right)\right. \\
&\left.-\left(m_{\Lambda_{c}^{*}}+m_{N}\right) \operatorname{Im}_{s} \Pi_{2}^{(i)}\left(s, q^{2}\right)\right] .
\end{aligned}
$$

In the case of the vector transition current, the same procedure of eliminating the $\Lambda_{c}^{*}$-contributions yields the following LCSR for the two most important form factors:

$$
\begin{gathered}
f_{1}\left(q^{2}\right)=\frac{e^{m_{\Lambda_{c}}^{2} / M^{2}}}{2 m_{\Lambda_{c}}\left(m_{\Lambda_{c}}+m_{\Lambda_{c}^{*}}\right) \lambda_{\Lambda_{c}}^{(i)}} \frac{1}{\pi} \int_{m_{c}^{2}}^{s_{0}} d s e^{-s / M^{2}}\left[( m _ { \Lambda _ { c } } + m _ { N } ) \left(\operatorname{Im}_{s} \tilde{\Pi}_{1}^{(i)}\left(s, q^{2}\right)\right.\right. \\
\left.\left.-\left(m_{\Lambda_{c}^{*}}-m_{N}\right) \operatorname{Im}_{s} \tilde{\Pi}_{2}^{(i)}\left(s, q^{2}\right)\right)+2 \operatorname{Im}_{s} \tilde{\Pi}_{3}^{(i)}\left(s, q^{2}\right)+2\left(m_{\Lambda_{c}^{*}}-m_{\Lambda_{c}}\right) \operatorname{Im}_{s} \tilde{\Pi}_{4}^{(i)}\left(s, q^{2}\right)\right] \\
f_{2}\left(q^{2}\right)=\frac{e^{m_{\Lambda_{c}}^{2} / M^{2}}}{2\left(m_{\Lambda_{c}}+m_{\Lambda_{c}^{*}}\right) \lambda_{\Lambda_{c}}^{(i)}} \frac{1}{\pi} \int_{m_{c}^{2}}^{s_{0}} d s e^{-s / M^{2}}\left[\operatorname{Im}_{s} \tilde{\Pi}_{1}^{(i)}\left(s, q^{2}\right)\right. \\
\left.-\left(m_{\Lambda_{c}^{*}}-m_{N}\right) \operatorname{Im}_{s} \tilde{\Pi}_{2}^{(i)}\left(s, q^{2}\right)-2 \operatorname{Im}_{s} \tilde{\Pi}_{4}^{(i)}\left(s, q^{2}\right)\right]
\end{gathered}
$$


The LCSR for the axial-vector form factors $g_{1}\left(q^{2}\right)$ and $g_{2}\left(q^{2}\right)$ can be obtained from the above sum rules for $f_{1}\left(q^{2}\right)$ and $f_{2}\left(q^{2}\right)$, respectively, by replacing $\operatorname{Im}_{s} \tilde{\Pi}_{j}^{(i)} \rightarrow \operatorname{Im}_{s} \bar{\Pi}_{j}^{(i)}$ and changing the sign of $m_{N}$. Furthermore, the LCSR obtained above are easily transformed to the case of $\Lambda_{b} \rightarrow p$ transition by replacing the $c$-quark by $b$-quark in the correlation function. Finally, to obtain LCSR for the $\Sigma_{c} \rightarrow p$ form factors we repeat the whole procedure for the correlation functions with the $\Sigma_{c}$-interpolation currents.

In practice, all three procedures: transformation to the dispersion integral form, subtraction of continuum and Borel transformation are unified in the following substitution rules for the integrals in (26), similar to the ones used in [8]:

$$
\begin{aligned}
\int d x \frac{\omega(x)}{D} \rightarrow & \int_{x_{0}}^{1} \frac{d x}{x} \omega(x) \exp \left(-\frac{s(x)}{M^{2}}\right) \\
\int d x \frac{\omega(x)}{D^{2}} \rightarrow & \frac{1}{M^{2}} \int_{x_{0}}^{1} \frac{d x}{x^{2}} \omega(x) \exp \left(-\frac{s(x)}{M^{2}}\right)+\frac{\omega\left(x_{0}\right) e^{-s_{0} / M^{2}}}{m_{c}^{2}+x_{0}^{2} m_{N}^{2}-q^{2}}, \\
\int d x \frac{\omega(x)}{D^{3}} \rightarrow & \frac{1}{2 M^{4}} \int_{x_{0}}^{1} \frac{d x}{x^{3}} \omega(x) \exp \left(-\frac{s(x)}{M^{2}}\right)+\frac{1}{2 M^{2}} \frac{\omega\left(x_{0}\right) e^{-s_{0} / M^{2}}}{x_{0}\left(m_{c}^{2}+x_{0}^{2} m_{N}^{2}-q^{2}\right)} \\
& -\left.\frac{1}{2} \frac{x_{0}^{2} e^{-s_{0} / M^{2}}}{\left(m_{c}^{2}+x_{0}^{2} m_{N}^{2}-q^{2}\right)} \frac{d}{d x}\left(\frac{\omega(x)}{x\left(m_{c}^{2}+x^{2} m_{N}^{2}-q^{2}\right)}\right)\right|_{x=x_{0}} .
\end{aligned}
$$

with $x_{0}=x\left(s_{0}\right)$ and for any numerator function $\omega(x)$ in (26). The surface terms appearing on r.h.s. of the above relations originate from the transformations of the integrals with the power of denominator $n=2,3$ to the "canonical" dispersion form with the first power of $s-(P-q)^{2}$ in denominator.

\section{Decay constants of charmed baryons}

To obtain decay constants of the $\Lambda_{c}$ baryon, the following two-point correlation function of the interpolating current and its Dirac-conjugate is considered:

$$
\begin{aligned}
F^{(i)}(q) & =i \int d^{4} z e^{i q \cdot z}\left\langle 0\left|T\left\{\eta_{\Lambda_{c}}^{(i)}(z), \bar{\eta}_{\Lambda_{c}}^{(i)}(0)\right\}\right| 0\right\rangle \\
& =F_{1}^{(i)}\left(q^{2}\right) \not q+F_{2}^{(i)}\left(q^{2}\right),
\end{aligned}
$$

where $i=\mathcal{P}, \mathcal{A}$, At $q^{2} \ll m_{c}^{2}$ this correlation function containing two invariant amplitudes is calculated from local OPE in terms of the perturbative and vacuum-condensate contributions up to dimension 6. The OPE results will be taken from the literature. Note that it is consistent to use two-point sum rules without the $\alpha_{s}$ corrections, since the latter are also not taken into account in LCSR. The hadronic dispersion relation for 
(36) reads:

$$
\begin{aligned}
F^{(i)}(q)= & \frac{\left|\lambda_{\Lambda_{c}}^{(i)}\right|^{2} m_{\Lambda_{c}}^{2}}{m_{\Lambda_{c}}^{2}-q^{2}}\left(\not q+m_{\Lambda_{c}}\right)+\frac{\left|\lambda_{\Lambda_{c}^{*}}^{(i)}\right|^{2} m_{\Lambda_{c}^{*}}^{2}}{m_{\Lambda_{c}^{*}}^{2}-q^{2}}\left(\not q-m_{\Lambda_{c}^{*}}\right) \\
& +\int_{\bar{s}_{0}^{h}}^{\infty} \frac{d s}{s-q^{2}}\left[\rho_{1}^{(i)}(s) \not q+\rho_{2}^{(i)}(s)\right] .
\end{aligned}
$$

Here again the $\Lambda_{c}^{*}$-contribution "contaminates" the sum rules. To tackle this problem we use the linear combination of the dispersion relations for the invariant amplitudes $F_{1}^{(i)}$ and $F_{2}^{(i)}$ and eliminate the $\Lambda_{c}^{*}$-state contribution, obtaining the following QCD sum rule:

$$
\left|\lambda_{\Lambda_{c}}^{(i)}\right|^{2}=\frac{e^{m_{\Lambda_{c}}^{2} / M_{2 p t}^{2}}}{m_{\Lambda_{c}}^{2}\left(m_{\Lambda_{c}}+m_{\Lambda_{c}^{*}}\right)} \frac{1}{\pi} \int_{m_{c}^{2}}^{s_{0}^{2 p t}} d s e^{-s / M_{2 p t}^{2}}\left[m_{\Lambda_{c}^{*}} \operatorname{Im} F_{1}^{(i)}(s)+\operatorname{Im} F_{2}^{(i)}(s)\right],
$$

where $M_{2 p t}^{2}$ and $s_{0}^{2 p t}$ are the Borel and threshold parameters.

For the decay constant $\lambda_{\Lambda_{c}}^{(\mathcal{P})}$ induced by the pseudoscalar interpolation current, the OPE results for the spectral densities of the invariant amplitudes $F_{1,2}^{(\mathcal{P})}$ are taken from [28] and presented in App. D. To access the decay constant of $\Lambda_{c}$ induced by the axial-vector current, we employ the results from [22] where a linear combination of two different interpolating currents $J_{\Lambda_{c}}^{(1)}+b J_{\Lambda_{c}}^{(2)}$ were used. At $b=-1 / 5$ we recover a superposition of the currents of our choice:

$$
J_{\Lambda}^{(1)}-\frac{1}{5} J_{\Lambda}^{(2)}=\frac{\sqrt{6}}{10}\left(\eta_{\Lambda_{c}}^{(\mathcal{A})}+4 \eta_{\Lambda_{c}}^{(\mathcal{P})}\right) .
$$

The correlation function of this combined current with its conjugate has a decomposition similar to (36), with two invariant amplitudes which we denote as $\tilde{F}_{1,2}$. From that we derive the sum rule for the linear combination of the decay constants:

$$
\left|\lambda_{\Lambda_{c}}^{(\mathcal{A})}+4 \lambda_{\Lambda_{c}}^{(\mathcal{P})}\right|^{2}=\frac{50 e^{m_{\Lambda_{c}}^{2} / M_{2 p t}^{2}}}{3 m_{\Lambda_{c}}^{2}\left(m_{\Lambda_{c}}+m_{\Lambda_{c}^{*}}\right)} \frac{1}{\pi} \int_{m_{c}^{2}}^{s_{0}^{2 p t}} d s e^{-s / M_{2 p t}^{2}}\left[m_{\Lambda_{c}^{*}} \operatorname{Im} \tilde{F}_{1}(s)+\operatorname{Im} \tilde{F}_{2}(s)\right]
$$

The OPE expressions for the spectral densities $\operatorname{Im} \tilde{F}_{1,2}(s)$ combined from the results obtained in 22] are collected in App. D. To resolve the ambiguity of the relative sign between $\lambda_{\Lambda_{c}}^{(\mathcal{A})}$ and $\lambda_{\Lambda_{c}}^{(\mathcal{P})}$ in this sum rule, we assume that they are of the same order of magnitude. The numerical analysis reveals that this is only possible if the two decay constants in (400) have the same sign. This allows us to obtain $\lambda_{\Lambda_{c}}^{(\mathcal{A})}$ using the value of $\lambda_{\Lambda_{c}}^{(\mathcal{P})}$ calculated above. 
Decay constants of the $\Sigma_{c}$ baryon have been calculated in Ref. [29] from the similar two-point QCD sum rules. Using Fierz transformation, we relate the mixed current $J_{\Sigma_{c}}$ used in that paper with a linear combination of the two currents of our choice:

$$
J_{\Sigma_{c}}=\left(u^{T} C \gamma_{5} c\right) u+b\left(u^{T} C c\right) \gamma_{5} u=\frac{1-b}{4} \eta_{\Sigma_{c}}^{(\mathcal{I})}+\frac{1+b}{8} \eta_{\Sigma_{c}}^{(\mathcal{T})}
$$

where $b$ is the mixing parameter. The following sum rule for the combination of decay constants is then obtained:

$$
\left|2(1-b) \lambda_{\Sigma_{c}}^{(\mathcal{I})}+(1+b) \lambda_{\Sigma_{c}}^{(\mathcal{T})}\right|^{2}=\frac{64 e^{m_{\Sigma_{c}}^{2}} / M_{2 p t}^{2}}{m_{\Sigma_{c}}^{2}\left(m_{\Sigma_{c}}+m_{\Sigma_{c}^{*}}\right)} \frac{1}{\pi} \int_{m_{c}^{2}}^{s_{0}^{2 p t}} d s e^{-s / M_{2 p t}^{2}}\left(m_{\Sigma^{*}} \operatorname{Im} \bar{F}_{1}(s)+\operatorname{Im} \bar{F}_{2}(s)\right)
$$

where the spectral densities $\bar{F}_{1}(s)$ and $\bar{F}_{2}(s)$ obtained from [29] are given in App. D. Choosing subsequently $b=1$ and $b=-1$ we obtain separate sum rule for $\lambda_{\Sigma_{c}}^{(\mathcal{I})}$ and for $\lambda_{\Sigma_{c}}^{(\mathcal{T})}$. Finally, the two-point QCD sum rules for the decay constants of $\Lambda_{b}$-baryon are obtained from the $\Lambda_{c}$-sum rules, replacing $c \rightarrow b$.

\section{LCSR for the strong couplings}

The strong coupling constants of $\Lambda_{c}$-baryon with nucleon and $D$ - or $D^{*}$-meson are formally defined as hadronic matrix elements:

$$
\begin{aligned}
\left\langle\Lambda_{c}(P-q) \mid D(-q) N(P)\right\rangle & =g_{\Lambda_{c} N D} \bar{u}_{\Lambda_{c}}(P-q) i \gamma_{5} u_{N}(P) \\
\left\langle\Lambda_{c}(P-q) \mid D^{*}(-q) N(P)\right\rangle & =\bar{u}_{\Lambda_{c}}(P-q)\left(g_{\Lambda_{c} N D^{*}}^{V} \phi+i \frac{g_{\Lambda_{c} N D^{*}}^{T}}{m_{\Lambda_{c}}+m_{N}} \sigma_{\mu \nu} \epsilon^{\mu} q^{\nu}\right) u_{N}(P)
\end{aligned}
$$

Different from the $D^{*} D \pi$ coupling that can be measured in kinematically allowed $D^{*} \rightarrow D \pi$ decays, a direct measurement of the baryonic strong couplings is not possible because at least one of the hadrons has to be off-shell. E.g., in the hadronic dispersion relation for the $\Lambda_{c} \rightarrow N$ form factor, the residue of the $D$ - or $D^{*}$-pole (for pseudoscalar or vector transition current) is proportional to the $\Lambda_{c} N D^{(*)}$ coupling. This pole is located at $q^{2}=m_{D^{(*)}}^{2}$, beyond the physical regions $q^{2} \leq\left(m_{\Lambda_{c}}-m_{N}\right)^{2}$ (for semileptonic decays and scattering) and $q^{2} \geq\left(m_{\Lambda_{c}}+m_{N}\right)^{2}$ (for the baryon pair production).

In the heavy mass limit for the $c$-quark we may obtain relations between the coupling constants appearing in (43). In this case the masses of the $\Lambda_{c}$ and $D^{(*)}$ become equal to $m_{c}$. The heavy mesons $D$ and $D^{*}$ form a spin symmetry doublet which can be represented by

$$
\mathcal{D}(v)=\mathcal{N}(1+\psi)\left(-i \gamma_{5} D+\gamma^{\mu} D_{\mu}^{*}\right) \quad \text { with } \quad v^{\mu} D_{\mu}^{*}=0
$$

where $D$ and $D^{*}$ represent the charmed meson fields and $\mathcal{N}$ is a normalization factor. Likewise, the spinor of the $\Lambda_{c}$ is equal to the $c$-quark spinor, since the light degrees of 
freedom are in a spinless state. Thus in the $m_{c} \rightarrow \infty$ limit, the two hadronic amplitudes in (43) can be unified in one:

$$
\mathcal{A}=\bar{u}_{\Lambda_{c}}(v) \mathcal{D}(v) \mathcal{M} u_{N}(P),
$$

with a Dirac-structure $\mathcal{M}$ and the nucleon bispinor $u_{N}$ accumulating the light degrees of freedom in the process. The quantity $\mathcal{M}$ may be expanded in the basis Dirac matrices 1 , $\gamma_{5}, \gamma_{\mu}, \gamma_{\mu} \gamma_{5}$ and $\sigma_{\mu \nu}$; due to parity and Lorentz invariance only the unit matrix remains. Hence we obtain

$$
\mathcal{A}=g \bar{u}_{\Lambda_{c}}\left(-i \gamma_{5} D+\gamma^{\mu} D_{\mu}^{*}\right) u_{N}(P),
$$

where $g$ is the strong coupling in the heavy quark limit. Comparing this with (43) we obtain the heavy mass relations

$$
g_{\Lambda_{c} N D}=-g_{\Lambda_{c} N D^{*}}^{V} \quad \text { and } \quad g_{\Lambda_{c} N D^{*}}^{T}=0 .
$$

Following the same procedure, one can also derive the following relation for the three strong couplings involving $\Sigma_{c}$ baryon:

$$
g_{\Sigma_{c} N D}+3 g_{\Sigma_{c} N D^{*}}^{V}=\frac{3 m_{\Sigma_{c}}+m_{N}-2 P \cdot v}{m_{\Sigma_{c}}+m_{N}} g_{\Sigma_{c} N D^{*}}^{T} .
$$

Here, the four-velocity vector is defined as $v=-q / m_{D}^{(*)}$; hence, up to $O\left(1 / m_{c}\right)$ corrections, $P \cdot v=\left(m_{\Sigma_{c}}^{2}-m_{D^{(*)}}^{2}\right) /\left(2 m_{D^{(*)}}\right)$.

The couplings (43) play an important role in various models of strong interactions formulated in terms of virtual hadron exchanges, like e.g., in the production of a charmed baryon pair in the $p \bar{p}$ collision, with a virtual $D^{(*)}$ exchange in $t$ - channel. In the forthcoming publication [30] we shall consider this process in the PANDA energy region. It is tempting to formulate the hadronic exchange models in terms of effective Lagrangians involving propagation and couplings of hadronic fields rather than quarks and gluons. An effective Lagrangian involving the hadronic couplings discussed above has the following form:

$$
\mathscr{L}_{\Lambda_{c} D^{(*)} N}=\bar{\Lambda}_{c}\left[i a_{\Lambda_{c} N D} \gamma_{5} D+\left(a_{\Lambda_{c} N D^{*}}^{V} \gamma^{\mu}+\frac{a_{\Lambda_{c} N D^{*}}^{T}}{m_{\Lambda_{c}}+m_{N}} \sigma^{\mu \nu} \partial_{\nu}\right) D_{\mu}^{*}\right] N+\text { h.c. }
$$

where we have defined new couplings $a_{i}$. However, these couplings are not necessarily the same as $g_{i}$ appearing in (43), since this depends on the kinematic region where the effective Lagrangian (49) is applied. The latter assumes point-like baryons, and hence it can only be used at impact parameters large compared to the size of the baryons, which means it is restricted to small momentum transfers $t=q^{2}$. This in turn means that the exchanged $D^{(*)}$ meson is far off shell and thus for such an application the couplings $a_{i}$ will be different from $g_{i}$. Nevertheless, one may use the coupling constants calculated here as an input for a more elaborated hadronic models based on Regge poles.

The possibility to calculate strong couplings from LCSR is based on the fact that they enter double dispersion relations for the same correlation function (11). E.g., to 
access the $\Lambda_{c} N D$ coupling, we employ the hadronic double dispersion relation for the correlation function $\Pi_{5}^{(i)}(P, q)$ with the pseudoscalar transition current (16), choosing one of the interpolating currents $i=\mathcal{P}, \mathcal{A}$. The double dispersion relation is obtained by analytically continuing the imaginary parts of the invariant amplitudes $\operatorname{Im}_{s} \Pi_{1,2}^{(i)}\left(s, q^{2}\right)$ in the second variable $q^{2}$. The result is given by the following expression containing the $\left\{\Lambda_{c}, D\right\}$ double pole (the ground-state contribution), and, in addition, the $\left\{\Lambda_{c}^{*}, D\right\}$ double pole:

$$
\begin{aligned}
\Pi_{5}^{(i)}(P, q)= & \frac{\lambda_{\Lambda_{c}}^{(i)} m_{D}^{2} f_{D} m_{\Lambda_{c}} g_{\Lambda_{c} N D}}{\left(m_{\Lambda_{c}}^{2}-(P-q)^{2}\right)\left(m_{D}^{2}-q^{2}\right)}\left[\left(m_{\Lambda_{c}}-m_{N}\right)-q\right] i \gamma_{5} u_{N}(P) \\
& +\frac{\lambda_{\Lambda_{c}^{*}}^{(i)} m_{D}^{2} f_{D} m_{\Lambda_{c}^{*}} g_{\Lambda_{c}^{*} N D}}{\left(m_{\Lambda_{c}^{*}}^{2}-(P-q)^{2}\right)\left(m_{D}^{2}-q^{2}\right)}\left[\left(m_{\Lambda_{c}^{*}}+m_{N}\right)+q\right] i \gamma_{5} u_{N}(P) \\
& +\ldots,
\end{aligned}
$$

where the ellipses indicate the contributions of excited and continuum states in both $\Lambda_{c}$ and $D$ channels which have a generic form of dispersion integrals over the hadronic double spectral density.

Similarly, for the $\Lambda_{c} D^{*} N$ couplings we employ the hadronic double dispersion relation for the correlation function with the vector transition current:

$$
\begin{aligned}
\Pi_{\mu}^{(i)}(P, q)= & \frac{m_{\Lambda_{c}} m_{D^{*}} f_{D^{*}} \lambda_{\Lambda_{c}}^{(i)}}{\left(m_{\Lambda_{c}}^{2}-(P-q)^{2}\right)\left(m_{D^{*}}^{2}-q^{2}\right)}\left\{-2 g_{\Lambda_{c} N D^{*}}^{V} P_{\mu}+2 \frac{g_{\Lambda_{c} N D^{*}}^{T}}{m_{\Lambda_{c}}+m_{N}} P_{\mu} \not q\right. \\
& -\left(g_{\Lambda_{c} N D^{*}}^{V}-g_{\Lambda_{c} N D^{*}}^{T}\right)\left[\left(m_{\Lambda_{c}}-m_{N}\right) \gamma_{\mu}+\gamma_{\mu} \not q\right] \\
& +\left[\left(2-\frac{m_{\Lambda_{c}}^{2}-m_{N}^{2}}{m_{D^{*}}^{2}}\right) g_{\Lambda_{c} N D^{*}}^{V}-g_{\Lambda_{c} N D^{*}}^{T}\right] q_{\mu} \\
& \left.+\left[-\frac{g_{\Lambda_{c} N D^{*}}^{T}}{m_{\Lambda_{c}}+m_{N}}+\frac{m_{\Lambda_{c}}-m_{N}}{m_{D^{*}}^{2}} g_{\Lambda_{c} N D^{*}}^{V}\right] q_{\mu} \not q\right\} u_{N}(P) \\
& +\frac{m_{\Lambda_{c}^{*}} m_{D^{*}} f_{D^{*}} \lambda_{\Lambda_{c}^{*}}^{(i)}}{\left(m_{\Lambda_{c}^{*}}^{2}-(P-q)^{2}\right)\left(m_{D^{*}}^{2}-q^{2}\right)}\left\{2 g_{\Lambda_{c}^{*} N D^{*}}^{V} P_{\mu}-2 \frac{g_{\Lambda_{c}^{*} N D^{*}}^{T}}{m_{\Lambda_{c}^{*}}+m_{N}} P_{\mu} \not q\right. \\
& -\left[g_{\Lambda_{c}^{*} N D^{*}}^{V}+\frac{m_{\Lambda_{c}^{*}}-m_{N}}{m_{\Lambda_{c}^{*}}+m_{N}} g_{\Lambda_{c}^{*} N D^{*}}^{T}\right]\left[\left(m_{\Lambda_{c}^{*}}+m_{N}\right) \gamma_{\mu}-\gamma_{\mu} \not q\right] \\
& +\left[\left(-2+\frac{m_{\Lambda_{c}^{*}}^{2}-m_{N}^{2}}{m_{D^{*}}^{2}}\right) g_{\Lambda_{c}^{*} N D^{*}}^{V}-\frac{m_{\Lambda_{c}^{*}}-m_{N}}{m_{\Lambda_{c}^{*}}+m_{N}} g_{\Lambda_{c}^{*} N D^{*}}^{T}\right] q_{\mu} \\
& \left.+\left[\frac{g_{\Lambda_{c}^{*} N D^{*}}^{T}}{m_{\Lambda_{c}^{*}}+m_{N}}+\frac{m_{\Lambda_{c}^{*}}+m_{N}}{m_{D^{*}}^{2}} g_{\Lambda_{c}^{*} N D^{*}}^{V}\right] q_{\mu} \not q\right\} u_{N}(P) \\
& +\ldots . .
\end{aligned}
$$

Decomposition of the relations (50) and (51) in terms of invariant amplitudes and elimination of the $\Lambda_{c}^{*}$ contributions are similar to the steps done in the derivation of the LCSR for the form factors. 
The OPE results for the invariant amplitudes obtained in Sect. 4 have now to be considered in the deep spacelike region for both variables $(P-q)^{2}, q^{2} \ll m_{c}^{2}$. The new elements needed for the quark-hadron duality approximation of the higher states are the double spectral densities of the invariant amplitudes. These amplitudes were already obtained in the form (26). It suffices to find double spectral representations for the master integrals of the type $\int d x x^{K} D^{-n}$ with $k \geq 0$ and $n=1,2,3$. For $n=1$, we obtain

$$
\begin{aligned}
\int_{0}^{1} d x \frac{x^{k}}{D}=\frac{1}{2 \pi} & \sum_{j=0}^{k} \int_{m_{c}^{2}}^{\infty} \frac{d s}{s-(P-q)^{2}} \int_{t_{1}(s)}^{t_{2}(s)} \frac{d s^{\prime}}{s^{\prime}-q^{2}}(-1)^{k+j / 2}\left[1+(-1)^{j}\right] \\
& \times \frac{1}{\left(2 m_{N}^{2}\right)^{k}} C_{k}^{j}\left(s-s^{\prime}-m_{N}^{2}\right)^{k-j}\left[\left(s^{\prime}-t_{1}\right)\left(t_{2}-s^{\prime}\right)\right]^{\frac{j-1}{2}}
\end{aligned}
$$

where $C_{k}^{j}$ are the binomial coefficients and the integration limits are

$$
t_{1,2}(s)=\left(s+m_{N}^{2}\right) \mp 2 m_{N} \sqrt{s-m_{c}^{2}} .
$$

The double spectral representations for the master integrals with $n=2$ and 3 , being more lengthy, are collected in App. E. Using these integrals it is easy to find the double dispersion representation for all integrals in (26), where the numerators depend polynomially on $x$, and linearly on $(P-q)^{2}$ and $q^{2}$, so that the latter variables can simply be replaced by $s$ and $s^{\prime}$ respectively.

Equating the OPE results to the double hadronic dispersion relations, adopting the quark-hadron duality approximation for the hadronic spectral densities and performing the double Borel transformation, $(P-q)^{2} \rightarrow M^{2}, q^{2} \rightarrow \widetilde{M}^{2}$, we derive LCSR for the three strong couplings of our interest:

$$
\begin{aligned}
& g_{\Lambda_{c} N D}=\frac{e^{m_{\Lambda_{c}}^{2} / M^{2}} e^{m_{D}^{2} / \widetilde{M}^{2}}}{m_{\Lambda_{c}}\left(m_{\Lambda_{c}}+m_{\Lambda_{c}^{*}}\right) m_{D}^{2} f_{D} \lambda_{\Lambda_{c}}^{(i)}} \frac{1}{\pi^{2}} \int_{m_{c}^{2}}^{s_{0}} d s e^{-s / M^{2}} \\
& \times \int_{t_{1}(s)}^{t_{2}(s)} d s^{\prime} e^{-s^{\prime} / \widetilde{M}} \operatorname{Im}_{s} \operatorname{Im}_{s^{\prime}}\left[\Pi_{1}^{(i)}\left(s, s^{\prime}\right)-\left(m_{\Lambda_{c}^{*}}+m_{N}\right) \Pi_{2}^{(i)}\left(s, s^{\prime}\right)\right], \\
& g_{\Lambda_{c} N D^{*}}^{V}=-\frac{e^{m_{\Lambda_{c}}^{2} / M^{2}} e^{m_{D^{*}}^{2} / \widetilde{M}^{2}}}{2 m_{\Lambda_{c}}\left(m_{\Lambda_{c}}+m_{\Lambda_{c}^{*}}\right) m_{D^{*}} f_{D^{*}} \lambda_{\Lambda_{c}}^{(i)}} \frac{1}{\pi^{2}} \int_{m_{c}^{2}}^{s_{0}} d s e^{-s / M^{2}} \\
& \times \int_{t_{1}(s)}^{t_{2}(s)} d s^{\prime} e^{-s^{\prime} / \tilde{M}^{2}} \operatorname{Im}_{s} \operatorname{Im}_{s^{\prime}}\left[\left(m_{\Lambda_{c}}+m_{N}\right)\left(\bar{\Pi}_{1}^{(i)}\left(s, s^{\prime}\right)-\left(m_{\Lambda_{c}^{*}}-m_{N}\right) \bar{\Pi}_{2}^{(i)}\left(s, s^{\prime}\right)\right)\right. \\
& \left.+2 \bar{\Pi}_{3}^{(i)}\left(s, s^{\prime}\right)+2\left(m_{\Lambda_{c}^{*}}-m_{\Lambda_{c}}\right) \bar{\Pi}_{4}^{(i)}\left(s, s^{\prime}\right)\right],
\end{aligned}
$$




$$
\begin{array}{r}
g_{\Lambda_{c} N D^{*}}^{T}=-\frac{\left(m_{\Lambda_{c}}+m_{N}\right) e^{m_{\Lambda_{c}}^{2} / M^{2}} e^{m_{D^{*}}^{2} / \widetilde{M}^{2}}}{2 m_{\Lambda_{c}}\left(m_{\Lambda_{c}}+m_{\Lambda_{c}^{*}}\right) m_{D^{*}} f_{D^{*}} \lambda_{\Lambda_{c}}^{(i)}} \frac{1}{\pi^{2}} \int_{m_{c}^{2}}^{s_{0}} d s e^{-s / M^{2}} \\
\times \int_{t_{1}(s)}^{t_{2}(s)} d s^{\prime} e^{-s^{\prime} / \widetilde{M}^{2}} \operatorname{Im}_{s} \operatorname{Im}_{s^{\prime}}\left[\bar{\Pi}_{1}^{(i)}\left(s, s^{\prime}\right)-\left(m_{\Lambda_{c}^{*}}-m_{N}\right) \bar{\Pi}_{2}^{(i)}\left(s, s^{\prime}\right)-2 \bar{\Pi}_{4}^{(i)}\left(s, s^{\prime}\right)\right] .
\end{array}
$$

The $D$ and $D^{*}$ decay constants entering these sum rules are defined in a standard way:

$$
\left\langle 0\left|m_{c} \bar{c} i \gamma_{5} u\right| D(p)\right\rangle=m_{D}^{2} f_{D}, \quad\left\langle 0\left|\bar{c} \gamma^{\mu} u\right| D^{*}(p, \epsilon)\right\rangle=m_{D^{*}} f_{D^{*}} \epsilon^{\mu} .
$$

Instead of fixing their numerical values we will use the two-point QCD sum rules for $f_{D^{(*)}}$ taken for consistency in $O\left(\alpha_{s}^{0}\right)$ (see e.g., [11]). The region in the $\left\{s, s^{\prime}\right\}$ plane occupied by the double spectral density of the correlation function calculated in OPE in the limit $m_{N} \rightarrow 0$, is reduced to the diagonal $s=s^{\prime}$, very similar to LCSR for $D^{*} D \pi$ coupling [11]. In that case it was sufficient to use one effective threshold $s_{0}$ for the duality approximation. Here we adopt a similar ansatz reflected in the integration limits in the above sum rules. Clearly, it is possible to use different borders of the duality region. The sensitivity of the LCSR results to this region is lowered by the Borel transformation, still an additional uncertainty is introduced. Hence, as far as the quark-hadron duality is concerned, the sum rules for the strong couplings are generally less accurate than the ones for the form factors.

\section{Numerical results}

We begin the numerical analysis with specifying the choice of the input for nucleon DA's, collected in App. A. Their normalization parameters have been calculated from two-point QCD sum rules [5]:

$$
\begin{array}{r}
f_{N}=(5.0 \pm 0.5) \times 10^{-3} \mathrm{GeV}^{2} \\
\lambda_{1}=-(27 \pm 9) \times 10^{-3} \mathrm{GeV}^{2}, \quad \lambda_{2}=(54 \pm 19) \times 10^{-3} \mathrm{GeV}^{2} .
\end{array}
$$

For the remaining five dimensionless parameters determining the shapes of the nucleon DA's, we use the model suggested in [8] in which the QCD 2-point sum rule estimates are adjusted, via LCSR, to the data on the nucleon electromagnetic form factors:

$$
A_{1}^{u}=0.13, \quad V_{1}^{d}=0.30, \quad f_{1}^{d}=0.33, \quad f_{1}^{u}=0.09, \quad f_{2}^{d}=0.25 .
$$

The masses of baryons are taken from [21]: $m_{N}=m_{p}=0.938 \mathrm{GeV}, m_{\Lambda_{c}}=2.286 \mathrm{GeV}$, $m_{\Lambda_{c}^{*}}=2.595 \mathrm{GeV}, m_{\Sigma_{c}}=2.454 \mathrm{GeV}, m_{\Sigma_{c}^{*}}=2.801 \mathrm{GeV}, m_{\Lambda_{b}}=5.620 \mathrm{GeV}$, and the estimated mass of the negative parity $b$-baryon: $m_{\Lambda_{b}^{*}}=5.85 \mathrm{GeV}$, is taken according to the QCD sum rule estimate [31].

For the virtual $c$ and $b$ quarks in the correlation functions, the $\overline{\mathrm{MS}}$ mass is preferable. We use $\bar{m}_{b}\left(\bar{m}_{b}\right)=4.16 \pm 0.03 \mathrm{GeV}, \bar{m}_{c}\left(\bar{m}_{c}\right)=1.28 \pm 0.03 \mathrm{GeV}$, taking the central 


\begin{tabular}{|c|c|c|}
\hline \hline QCD sum rule & Borel parameter $\left(\mathrm{GeV}^{2}\right)$ & eff. threshold $\left(\mathrm{GeV}^{2}\right)$ \\
\hline$L C S R$, form factor & $M^{2}$ & $s_{0}$ \\
$\Lambda_{c}\left(\Sigma_{c}\right) \rightarrow p$ & $7.5 \pm 2.5$ & $10.0 \pm 0.5$ \\
$\Lambda_{b} \rightarrow p$ & $20.0 \pm 5.0$ & $40.0 \pm 1.0$ \\
\hline LCSR, strong coupling & $M^{2} \widetilde{M}^{2}$ & $s_{0}$ \\
$\Lambda_{c}\left(\Sigma_{c}\right) N D$ & $4.5 \pm 1.5$ & \} $10.0 \pm 0.5$ \\
& \} $7.5 \pm 2.5$ & \multirow{2}{*}{$s_{0}^{2 p t}$} \\
$\Lambda_{c}\left(\Sigma_{c}\right) N D^{*}$ & $5.0 \pm 1.5$ & $10.0 \pm 0.5$ \\
2-point SR, decay constant & $M_{2 p t}^{2}$ & $40.0 \pm 1.0$ \\
$\Lambda_{c}\left(\Sigma_{c}\right)$ & $2.5 \pm 0.5$ & $6.5 \pm 0.5$ \\
$\Lambda_{b}$ & $5.0 \pm 1.0$ & $8.0 \pm 0.5$ \\
$D$ & $2.0 \pm 0.5$ & \\
$D^{*}$ & $2.0 \pm 0.5$ & \\
\hline \hline
\end{tabular}

Table 1: Borel parameters and effective thresholds used in various sum rules.

values from the precise determination 32 based on the quarkonium sum rules and twice inflating the uncertainties. In the absence of gluon corrections the only renormalization scale which enters our calculation is the factorization scale $\mu$ of the nucleon DA's. We adopt the same scale for the quark masses, using the intervals $\mu_{c}=1.5 \pm 0.5 \mathrm{GeV}$ and $\mu_{b}=4.0 \pm 1.0 \mathrm{GeV}$ for LCSR with $c$ and $b$ quarks, respectively. The evolution of the scale dependent parameters in the nucleon DA's is taken according to [26]. We also adopt the same vacuum condensates as in [33, 34, in particular, the quark condensate density $\langle\bar{q} q\rangle(1 \mathrm{GeV})=-\left(246_{-19}^{+28} \mathrm{MeV}\right)^{3}$.

The intervals of Borel parameters used in the sum rules considered here are listed in Table 1. Their choice is based on the usual criteria, that is, both power corrections and continuum contributions in the sum rules have to be sufficiently suppressed. The corresponding effective thresholds (see Table 11) are adjusted so that the differentiated sum rules reproduce the measured mass of the lowest baryon or meson with at least a $10 \%$ accuracy.

Furthermore, instead of substituting in LCSR a certain fixed value for the $\Lambda_{c(b)}$ or $\Sigma_{c}$ decay constants, we use the corresponding two-point sum rules. This somewhat reduces the overall uncertainties. Still, to give an idea of the magnitude of the decay constants, let us quote their numerical values:

$$
\lambda_{\Lambda_{c}}^{(\mathcal{A})}=1.51_{-0.39}^{+0.37} \times 10^{-2} \mathrm{GeV}^{2}, \quad \lambda_{\Lambda_{c}}^{(\mathcal{P})}=1.19_{-0.28}^{+0.19} \times 10^{-2} \mathrm{GeV}^{2},
$$




\begin{tabular}{|c|c|c|c|c|}
\hline Current & $\eta_{\Lambda_{c}}^{(\mathcal{A})}$ & $\eta_{\Lambda_{c}}^{(\mathcal{P})}$ & $\eta_{\Sigma_{c}}^{(\mathcal{I})}$ & $\eta_{\Sigma_{c}}^{(\mathcal{T})}$ \\
\hline Form factor & \multicolumn{2}{|c|}{$\Lambda_{c} \rightarrow p$} & \multicolumn{2}{|c|}{$\Sigma_{c} \rightarrow p$} \\
\hline$G(0)$ & $0.39_{-0.09}^{+0.11}$ & $0.48_{-0.13}^{+0.13}$ & $0.066_{-0.032}^{+0.035}$ & $0.061_{-0.011}^{+0.011}$ \\
\hline$f_{1}(0)$ & $0.46_{-0.11}^{+0.15}$ & $0.59_{-0.16}^{+0.15}$ & $-0.22_{-0.07}^{+0.07}$ & $-0.23_{-0.05}^{+0.04}$ \\
\hline$f_{2}(0)$ & $-0.32_{-0.07}^{+0.08}$ & $-0.43_{-0.12}^{+0.13}$ & $-0.24_{-0.05}^{+0.05}$ & $-0.25_{-0.06}^{+0.06}$ \\
\hline$g_{1}(0)$ & $0.49_{-0.11}^{+0.14}$ & $0.55_{-0.15}^{+0.14}$ & $0.11_{-0.05}^{+0.05}$ & $0.060_{-0.008}^{+0.007}$ \\
\hline$g_{2}(0)$ & $-0.20_{-0.06}^{+0.09}$ & $-0.16_{-0.05}^{+0.08}$ & $-0.002_{-0.044}^{+0.054}$ & $-0.030_{-0.039}^{+0.039}$ \\
\hline Strong coupling & \multicolumn{2}{|c|}{$\Lambda_{c} D^{(*)} N$} & \multicolumn{2}{|c|}{$\Sigma_{c} D^{(*)} N$} \\
\hline$g_{\Lambda_{c}\left(\Sigma_{c}\right) N D}$ & $13.8_{-4.1}^{+5.2}$ & $10.7_{-4.3}^{+5.3}$ & $1.3_{-0.9}^{+1.0}$ & $1.3_{-0.8}^{+1.2}$ \\
\hline$g_{\Lambda_{c}\left(\Sigma_{c}\right) N D^{*}}^{V}$ & $-7.9_{-3.3}^{+2.7}$ & $-5.8_{-2.5}^{+2.1}$ & $1.0_{-0.6}^{+1.3}$ & $0.74_{-0.45}^{+1.08}$ \\
\hline$g_{\Lambda_{c}\left(\Sigma_{c}\right) N D^{*}}^{T}$ & $4.7_{-2.0}^{+2.7}$ & $3.6_{-1.8}^{+2.9}$ & $2.1_{-1.0}^{+1.9}$ & $1.8_{-0.8}^{+1.6}$ \\
\hline
\end{tabular}

Table 2: Numerical results for the transition form factors and strong couplings of charmed baryons obtained from LCSR with different interpolating currents.

$$
\begin{array}{lll}
\lambda_{\Lambda_{b}}^{(\mathcal{A})}=1.27_{-0.34}^{+0.35} \times 10^{-2} \mathrm{GeV}^{2}, & \lambda_{\Lambda_{b}}^{(\mathcal{P})}=1.09_{-0.30}^{+0.31} \times 10^{-2} \mathrm{GeV}^{2} \\
\lambda_{\Sigma_{c}}^{(\mathcal{I})}=3.08_{-0.74}^{+0.49} \times 10^{-2} \mathrm{GeV}^{2}, & \lambda_{\Lambda_{c}}^{(\mathcal{T})}=6.08_{-1.48}^{+0.90} \times 10^{-2} \mathrm{GeV}^{2} .
\end{array}
$$

After specifying all input parameters, we compute the numerical values of the $\Lambda_{c}\left(\Sigma_{c}\right) \rightarrow p$ form factors at $q^{2}=0$ and the $\Lambda_{c}\left(\Sigma_{c}\right) N D^{(*)}$ strong couplings. The results are collected in Table 2, where the total uncertainties are estimated by varying separate input parameters within their ranges and adding the resulting separate uncertainties of the form factors and strong couplings in quadrature. Correlations between different form factors and strong couplings with respect to the input variation make the sum rule predictions for the ratios of these hadronic matrix elements even more accurate.

Replacing $c$-quark with the $b$-quark in LCSR we calculate the phenomenologically important $\Lambda_{b} \rightarrow p$ form factors. They are collected in Table 3. In this case, not only the 


\begin{tabular}{|c|c|c|}
\hline \hline form factors & $\eta_{\Lambda_{b}}^{(\mathcal{A})}$ & $\eta_{\Lambda_{b}}^{(\mathcal{P})}$ \\
\hline$f_{1}(0)$ & $0.14_{-0.03}^{+0.03}$ & $0.12_{-0.04}^{+0.03}$ \\
$b_{1}$ & $-1.49_{-1.88}^{+1.68}$ & $-9.13_{-1.12}^{+0.88}$ \\
$f_{2}(0)$ & $-0.054_{-0.013}^{+0.016}$ & $-0.047_{-0.013}^{+0.015}$ \\
$b_{2}$ & $-14.0_{-1.8}^{+1.2}$ & $-18.5_{-2.0}^{+1.7}$ \\
\hline$g_{1}(0)$ & $0.14_{-0.03}^{+0.03}$ & $0.12_{-0.03}^{+0.03}$ \\
$\tilde{b}_{1}$ & $-4.05_{-1.81}^{+1.38}$ & $-9.18_{-1.06}^{+0.75}$ \\
$g_{2}(0)$ & $-0.028_{-0.009}^{+0.012}$ & $-0.016_{-0.005}^{+0.007}$ \\
$\tilde{b}_{2}$ & $-20.2_{-2.1}^{+1.0}$ & $-22.5_{-1.7}^{+1.3}$ \\
\hline \hline
\end{tabular}

Table 3: Numerical results of $\Lambda_{b} \rightarrow p$ transition form factors at zero momentum transfer and their slope parameters obtained from LCSR with different interpolating currents.

zero momentum transfer but also small and intermediate $q^{2} \ll m_{b}^{2}$ are available from LCSR. We estimate the maximal value of $q^{2}$ accessible with LCSR to lie in the interval $q^{2}=11-15 \mathrm{GeV}^{2}$ and adopt, conservatively, the lowest value $q_{\max }^{2}=11 \mathrm{GeV}^{2}$. At larger $q^{2}$ light-cone OPE is not reliable, in particular the contribution of the highest twist- 6 nucleon DA's starts to grow with respect to the lower twists. Note that the contributions of all twist 3,4, 5 components of nucleon DA's are numerically important in LCSR.

Several additional comments on the numerical results obtained above are in order.

- We found that form factors and strong couplings are (within uncertainties) insensitive to the interpolating current of the heavy baryon, once the contribution of the negative-parity baryon is included in the hadronic dispersion relation. We have checked that if the negative-parity baryon is simply absorbed in the dualityapproximated continuum, the sum rules yield numerical predictions that are considerably more sensitive to the choice of the interpolating current.

- The achieved accuracy of LCSR for the form factors of heavy baryons is well illustrated by the equation-of-motion relation (13) which yields $G(0)=g_{1}(0)$. Comparing the numerical results for both form factors calculated from two different 
LCSR, we see that this relation is violated numerically at the level of $20 \%$ which is also in the ballpark of the estimated uncertainty of the LCSR.

- In the infinitely heavy quark limit, the relation $f_{1}\left(q^{2}\right)=g_{1}\left(q^{2}\right)$ is valid for $\Lambda_{Q} \rightarrow N$ form factors and it is well reproduced by our numerical results for both $Q=c, b$. Note that this relation holds for any $q^{2}$, since only the heavy-quark spin symmetry is employed in its derivation.

- The heavy-mass relations for the three strong couplings of $\Lambda_{c}$ baryon, shown in (47), are only qualitatively supported by the LCSR predictions obtained for the finite $c$ quark mass. In particular, the magnitude of $g_{\Lambda_{c} N D^{*}}^{T}$ characterizes the size of $1 / m_{c}$ correction. Interestingly, the results for $\Sigma_{c} N D^{(*)}$ couplings are in a better agreement with the heavy mass relation (48).

Concluding this section we make a few comments on the earlier sum rule calculations of the heavy-baryon form factors and couplings in the literature.

The $\Lambda_{b} \rightarrow p$ form factors were calculated [18 in a different approach, using three-point QCD sum rules (see also [20]), where $\Lambda_{b}$ was interpolated by the pseudoscalar current and the tensor interpolating current for the nucleon was adopted. The form factors predicted in [18] are in agreement with our results. However, the other interpolating currents, as well as the role of negative-parity partners of both $\Lambda_{b}$ and proton remain obscure, bringing unaccounted uncertainties in the numerical predictions.

The strong coupling $g_{\Lambda_{c} N D}$ was calculated from three-point QCD sum rules in Ref. [19]. In fact this approach radically differs from the one we use here, first of all, in the definition of the coupling itself. The starting point is the correlation function with two baryon interpolating currents and one pseudoscalar transition current. The latter is then simply replaced by the $D$ meson. The relation of this definition of the strong coupling to the one used here is difficult to assess. Again, the problem of negative-parity baryons in both nucleon and $\Lambda_{c}$ channels was practically ignored absorbing these states into the hadronic continuum.

Light-cone sum rules in HQET with $\Lambda_{b}$-distribution amplitudes worked out in [35] was employed in [14 to calculate the $\Lambda_{b} \rightarrow p$ form factors. The nucleon was interpolated by the CZ current $(u C \not u) \gamma_{5} \not d$ suggested in [36]. The form factor $f_{1}(0)$ obtained in [14] is about an order of magnitude smaller than the one obtained here from LCSR with nucleon distribution amplitudes. Note that the $\mathrm{CZ}$ current can also couple to $I=3 / 2$ and $J=3 / 2$ states so that the sum rules for the $\Lambda_{b} \rightarrow p$ transition form factors are probably influenced by large and unaccounted $\Delta$ - resonance contribution. It is known [8] that, e.g., the isospin relations between nucleon form factors are violated when one uses LCSR with CZ current. Note that a similar current was employed also in [12, 13].

The $\Lambda_{b, c} \rightarrow N$ transitions were investigated in [15, 16] using LCSR with the nucleon DA's. As opposed to our choice, the most general interpolating current for the $\Lambda_{b, c}$ baryon was employed introducing an arbitrary parameter $\beta$ for the mixing of different components in this current. The stability of the calculated form factors with respect to the variation of $\beta$ was used as a criterion for choosing a working interval of $\beta$. In our opinion, such a procedure introduces a sort of a new systematic error related with 
the choice of the mixing parameter. Most importantly, the problem of separating the negative parity baryon contributions in LCSR remains unsolved, because the latter are again attributed to the continuum estimated with the usual quark-hadron duality ansatz. This may explain the substantial difference of our predictions from the ones presented in [15].

\section{Applications to exclusive $\Lambda_{b}$ decays}

With the results for the $\Lambda_{b} \rightarrow p$ form factors obtained from LCSR we are now in a position to predict the differential decay distribution for the exclusive semileptonic $\Lambda_{b} \rightarrow p \ell \nu_{\ell}$ decay, which is a $b \rightarrow u$ transition with the CKM parameter $V_{u b}$. In the massless lepton approximation, the form factors $f_{3}\left(q^{2}\right)$ and $g_{3}\left(q^{2}\right)$ do not contribute to the decay width, hence it is sufficient to use the results for the four form factors $f_{1,2}$ and $g_{1,2}$ given in the previous section. Since the form factors are only available at $q^{2} \leq q_{\max }^{2}$, we apply the conformal mapping $q^{2} \rightarrow z$ and $z$-series parametrization to extrapolate the form factors to the whole semileptonic region $q^{2} \leq\left(m_{\Lambda_{b}}-m_{N}\right)^{2}$. More specifically, we use the $z$-series parametrization in the BCL-version suggested in [37. The mapping transformation reads:

$$
z\left(q^{2}, t_{0}\right)=\frac{\sqrt{t_{+}-q^{2}}-\sqrt{t_{+}-t_{0}}}{\sqrt{t_{+}-q^{2}}+\sqrt{t_{+}-t_{0}}},
$$

where $t_{ \pm}=\left(m_{\Lambda_{b}} \pm m_{N}\right)^{2}$, and $t_{0}=t_{+}-\sqrt{t_{+}-t_{-}} \sqrt{t_{+}-t_{\min }}$ is chosen to maximally reduce the interval of $z$ obtained after the mapping of the interval $t_{\min }<q^{2}<t_{-}$, where $t_{\min }=q_{\min }^{2}<q^{2}<q_{\max }^{2}$ is the LCSR validity region. In the numerical analysis, $t_{\min }=-6 \mathrm{GeV}^{2}$ is adopted. Furthermore, we employ the following parametrization

$$
\begin{aligned}
& f_{i}\left(q^{2}\right)=\frac{f_{i}(0)}{1-q^{2} / m_{B^{*}\left(1^{-}\right)}^{2}}\left\{1+b_{i}\left(z\left(q^{2}, t_{0}\right)-z\left(0, t_{0}\right)\right)\right\}, \\
& g_{i}\left(q^{2}\right)=\frac{g_{i}(0)}{1-q^{2} / m_{B^{*}\left(1^{+}\right)}^{2}}\left\{1+\tilde{b}_{i}\left(z\left(q^{2}, t_{0}\right)-z\left(0, t_{0}\right)\right)\right\} .
\end{aligned}
$$

where $i=1,2, m_{B^{*}\left(1^{-}\right)}=5.325 \mathrm{GeV}$ and $m_{B^{*}\left(1^{+}\right)}=5.723 \mathrm{GeV}$ [21]. Fitting the shape parameters $b_{i}$ and $\tilde{b}_{i}$ to LCSR predictions at $q^{2} \leq q_{\text {max }}^{2}$, we obtained the $\Lambda_{b} \rightarrow p$ form factors shown in Fig. 2. The shape parameters (see Table 3) turn out to be more sensitive to the choice of the interpolating current, also the uncertainties of these parameters (correlated with the uncertainties of the form factor normalization) are larger. This however plays role only at large $q^{2}$, beyond the region of validity of LCSR.

Finally, we calculate the differential width of $\Lambda_{b} \rightarrow p \ell \nu$ decay using the following 

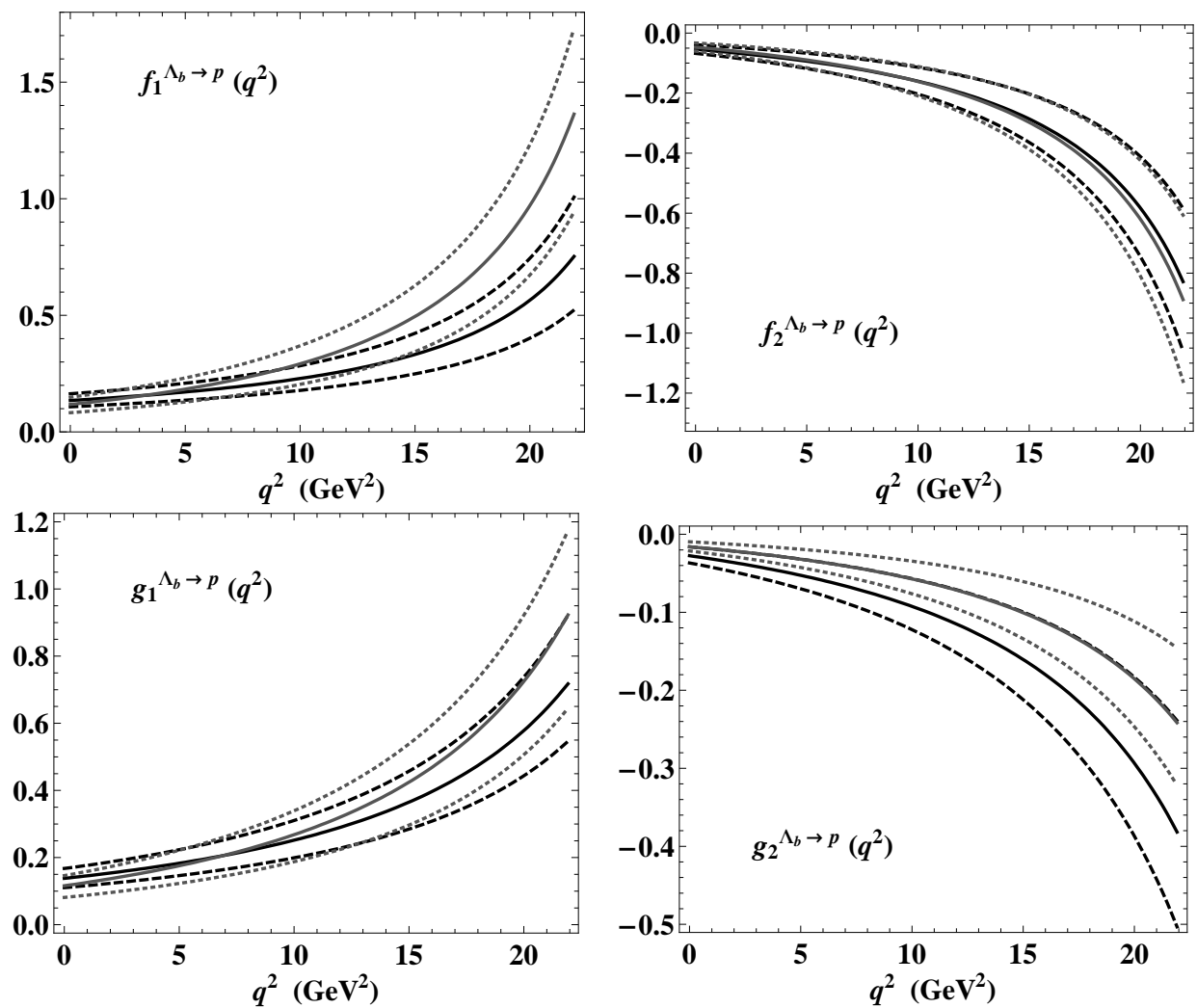

Figure 2: $\Lambda_{b} \rightarrow p$ transition form factors obtained from LCSR at $q^{2} \leq 11 \mathrm{GeV}^{2}$ and extrapolated to larger $q^{2}$ using the series-parametrization: black (grey) solid lines correspond to the axial-vector (pseudoscalar) interpolating current for $\Lambda_{b}$, black long-dashed (grey short-dashed) lines indicate uncertainties.

expression.

$$
\begin{array}{r}
\frac{d \Gamma}{d q^{2}}\left(\Lambda_{b} \rightarrow p l \nu_{l}\right)=\frac{G_{F}^{2} m_{\Lambda_{b}}^{3}}{192 \pi^{3}}\left|V_{u b}\right|^{2} \lambda^{1 / 2}\left(1, r^{2}, t\right)\left\{\left[(1-r)^{2}-t\right]\left[(1+r)^{2}+2 t\right]\left|f_{1}\left(q^{2}\right)\right|^{2}\right. \\
+\left[(1+r)^{2}-t\right]\left[(1-r)^{2}+2 t\right]\left|g_{1}\left(q^{2}\right)\right|^{2}-6 t\left[(1-r)^{2}-t\right](1+r) f_{1}\left(q^{2}\right) f_{2}\left(q^{2}\right) \\
-6 t\left[(1+r)^{2}-t\right](1-r) g_{1}\left(q^{2}\right) g_{2}\left(q^{2}\right)+t\left[(1-r)^{2}-t\right]\left[2(1+r)^{2}+t\right]\left|f_{2}\left(q^{2}\right)\right|^{2} \\
\left.+t\left[(1+r)^{2}-t\right]\left[2(1-r)^{2}+t\right]\left|g_{2}\left(q^{2}\right)\right|^{2}\right\}
\end{array}
$$

where $r=m_{N} / m_{\Lambda_{b}}, t=q^{2} / m_{\Lambda_{b}}^{2}$ and $\lambda(a, b, c)=a^{2}+b^{2}+c^{2}-2 a b-2 a c-2 b c$. Substituting the form factors (62) and integrating over $q^{2}$ we obtain the total branching fraction

$$
B R\left(\Lambda_{b} \rightarrow p l \nu_{l}\right)=\left\{\begin{array}{c}
\left(\left.3.3_{-1.2}^{+1.5}\right|_{t h .} \pm\left. 0.1\right|_{\text {exp. }}\right) \\
\left(\left.4.0_{-2.0}^{+2.3}\right|_{\text {th. }} \pm\left. 0.1\right|_{\text {exp. }}\right)
\end{array}\right\}\left(\frac{\left|V_{u b}\right|}{3.5 \cdot 10^{-3}}\right)^{2} \times 10^{-4}
$$


where the upper (lower) interval corresponds to the form factors obtained from LCSR with the axial-vector (pseudoscalar) $\Lambda_{b}$-interpolating current, and the lifetime $\tau_{\Lambda_{b}}=$ $\left(1.391_{-0.037}^{+0.038}\right)$ ps from [21] is used. The normalized $q^{2}$ distribution is plotted in Fig. 3. The enhancement in the region of large $q^{2}$ due to the growth of the form factors is in this case quite pronounced because the width of this decay contains only the $S$-wave phasespace factor $\lambda^{1 / 2}$, as opposed to the $B \rightarrow \pi l \nu$ decay width, where there is a $P$-wave factor $\lambda^{3 / 2}$.

Following our recent analysis of semileptonic $B \rightarrow \pi l \nu$ decay [38], we also calculate the specific integral

$$
\Delta \zeta\left(0, q_{\max }^{2}\right)=\frac{1}{\left|V_{u b}\right|^{2}} \int_{0}^{q_{\max }^{2}} d q^{2} \frac{d \Gamma}{d q^{2}}\left(\Lambda_{b} \rightarrow p l \nu_{l}\right),
$$

where the form factors directly calculated from LCSR are used, independent of their parametrization and/or extrapolation. Our prediction for the above integral from LCSR with the axial-vector $\Lambda_{b^{-}}$interpolating current is :

$$
\begin{aligned}
\Delta \zeta\left(0,11 \mathrm{GeV}^{2}\right)= & \left.\left.\left.\left.\left.\left.\left.5.5_{-0.9}^{+1.1}\right|_{f_{N}}{ }_{-0.4}^{+0.5}\right|_{\lambda_{1}}{ }_{-0.0}^{+0.1}\right|_{m_{b}}{ }_{-0.1}^{+0.0}\right|_{\mu}{ }_{-0.2}^{+0.5}\right|_{M^{2}}{ }_{-0.4}^{+0.5}\right|_{s_{0}}{ }_{-0.4}^{+0.4}\right|_{M_{2 p t}^{2}} \\
& \left.\left.{ }_{-0.0}^{+0.1}\right|_{s_{0}^{2 p t}}{ }_{-1.7}^{+2.1}\right|_{\langle q \bar{q}\rangle} \mathrm{ps}^{-1}=5.5_{-2.0}^{+2.5} \mathrm{ps}^{-1},
\end{aligned}
$$

where also the uncertainties due to the variations of separate input parameters are shown (only those which are larger than $O(1 \%)$ ). The total error quoted above is obtained by adding all separate uncertainties in quadrature. A very close interval is obtained in the case of the pseudoscalar $\Lambda_{b}$-interpolating current:

$$
\begin{aligned}
\Delta \zeta\left(0,11 \mathrm{GeV}^{2}\right)= & \left.\left.\left.\left.\left.\left.\left.5.6_{-1.7}^{+2.0}\right|_{\lambda_{1}}{ }_{-1.9}^{+2.3}\right|_{\lambda_{2}}{ }_{-0.1}^{+0.0}\right|_{m_{b}}{ }_{-0.4}^{+0.2}\right|_{\mu}{ }_{-0.2}^{+0.6}\right|_{M^{2}}{ }_{-0.3}^{+0.3}\right|_{s_{0}}{ }_{-1.2}^{+0.4}\right|_{M_{2 p t}^{2}} \\
& \left.\left.{ }_{-0.3}^{+0.3}\right|_{s_{0}^{2 p t}}{ }_{-1.2}^{+0.7}\right|_{\langle q \bar{q}\rangle} \mathrm{ps}^{-1}=5.6_{-2.9}^{+3.2} \mathrm{ps}^{-1} .
\end{aligned}
$$

Note that the accuracy of this prediction is not yet competitive with the one for $B \rightarrow \pi \ell \nu_{l}$ [38. Still the exclusive semileptonic decay of $\Lambda_{b}$ offers a possibility of $\left|V_{u b}\right|$ determination independent of the $B$-meson semileptonic decays.

Another interesting possibility to use the $\Lambda_{b} \rightarrow p$ form factors, is to estimate the rate of the nonleptonic $\Lambda_{b} \rightarrow p \pi$ decay in the factorization approximation and to compare the result with the recently measured branching fraction. We write down the factorizable amplitude in the same form as in [39]:

$$
\begin{array}{r}
\mathcal{M}_{f}\left(\Lambda_{b} \rightarrow p \pi\right)=\frac{G_{F}}{\sqrt{2}} f_{\pi} \bar{u}_{N}\left\{\left[V_{u b} V_{u d}^{*} a_{1}-V_{t b} V_{t d}^{*}\left(a_{4}+a_{10}+R_{1}^{\pi}\left(a_{6}+a_{8}\right)\right)\right]\right. \\
{\left[f_{1}\left(m_{\pi}^{2}\right)\left(m_{\Lambda_{b}}-m_{N}\right)-f_{3}\left(m_{\pi}^{2}\right) m_{\pi}^{2}\right]+\left[V_{u b} V_{u d}^{*} a_{1}-V_{t b} V_{t d}^{*}\left(a_{4}+a_{10}-R_{2}^{\pi}\left(a_{6}+a_{8}\right)\right)\right]} \\
\left.\left[g_{1}\left(m_{\pi}^{2}\right)\left(m_{\Lambda_{b}}+m_{N}\right)-g_{3}\left(m_{\pi}^{2}\right) m_{\pi}^{2}\right] \gamma_{5}\right\} u_{\Lambda_{b}},
\end{array}
$$

where $a_{i}$ are the combinations of Wilson coefficients of the effective weak Hamiltonian and

$$
R_{1}^{\pi}=\frac{2 m_{\pi}^{2}}{\left(m_{b}-m_{u}\right)\left(m_{u}+m_{d}\right)}, \quad R_{2}^{\pi}=\frac{2 m_{\pi}^{2}}{\left(m_{b}+m_{u}\right)\left(m_{u}+m_{d}\right)} .
$$




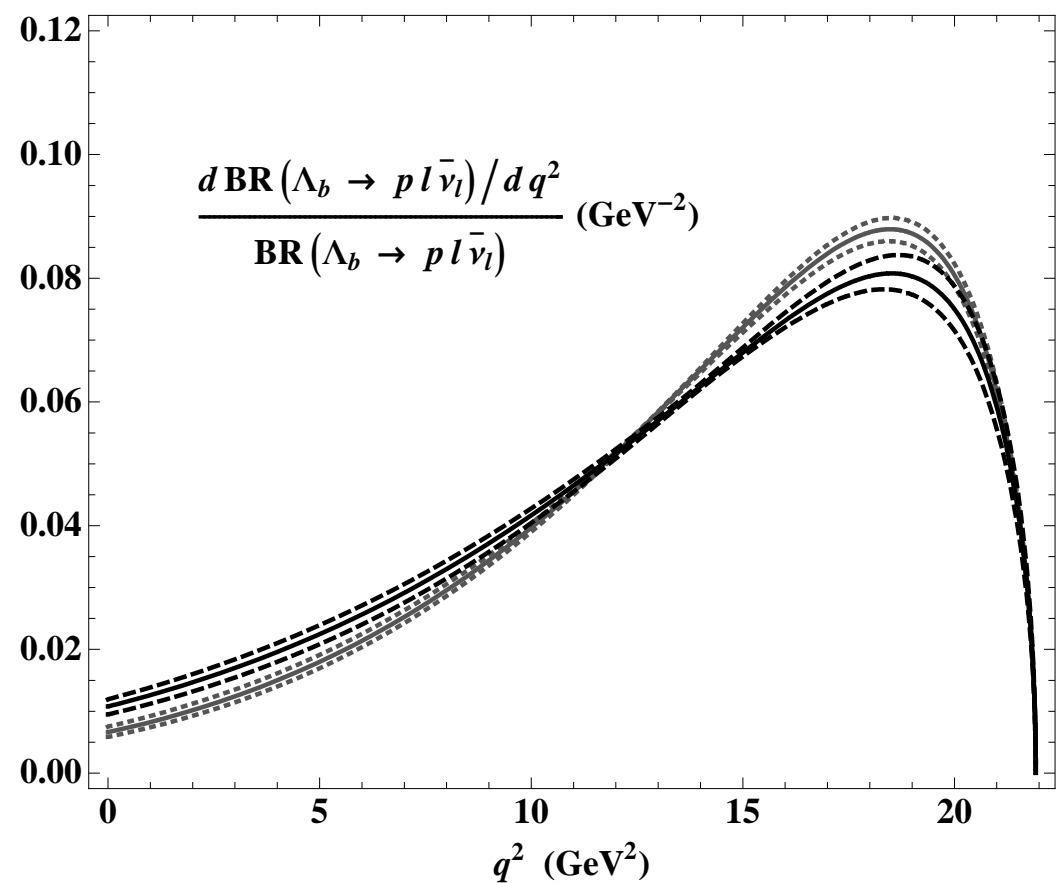

Figure 3: Normalized differential width of $\Lambda_{b} \rightarrow p \ell \nu_{\ell}$ calculated with the form factors obtained from LCSR and extrapolated to larger $q^{2}$ using the seriesparametrization. The notation is the same as in Fig. Q2.

The pion mass is neglected, hence we only use $f_{1}(0)$ and $g_{1}(0)$ in the numerical analysis. Our prediction for the branching fraction:

$$
\operatorname{BR}\left(\Lambda_{b} \rightarrow p \pi\right) \times 10^{6}=3.8_{-1.0}^{+1.3}\left(2.8_{-0.9}^{+1.1}\right),
$$

obtained with the axial-vector (pseudoscalar) interpolating current is consistent with the experimental measurement [40] $\mathrm{BR}\left(\Lambda_{b} \rightarrow p \pi\right) \times 10^{6}=3.5 \pm 0.6 \pm 0.9$, indicating that nonfactorizable contributions in this decay are at least moderate. This is in agreement with the expectations based on QCD factorization for a nonleptonic decay of a heavy hadron in two light hadrons. On the other hand, our prediction is in a sharp contrast to the analysis of $\Lambda_{b} \rightarrow p \pi$ done in [39] where the $k_{T}$-factorization approach was applied to the $\Lambda_{b} \rightarrow p$ form factors in the "conventional PQCD" scenario, hence the soft form factors were not taken into account. As a result the form factors predicted in that approach are almost two orders of magnitude smaller than the ones obtained from LCSR. On the contrary, the nonfactorizable contributions in $\Lambda_{b} \rightarrow p \pi$ calculated in [39] applying the same PQCD approach, are considerably enhanced, so that their predicted branching fraction is also in the same ballpark as the experimental one.

Further applications to semileptonic, radiative and nonleptonic decays of heavy baryons demand also the $\Lambda_{c, b} \rightarrow \Lambda$ form factors. In this respect our results can only be used in the $S U(3)_{\text {flavour }}$ approximation. 


\section{Discussion}

In this paper, we considered the form factors and strong couplings of heavy baryons. These non-perturbative quantities are important inputs for a rich variety of phenomenological applications, from the $\Lambda_{b, c}$ (electro)weak decays relevant for the flavour physics to the estimates of charmed hadron production. The calculation we have done is based on the method of QCD LCSR, where we use the light-cone DA's of the nucleon and interpolate the heavy baryons by appropriate currents. These sum rules are more complicated than the ones for the meson form factors and strong couplings. First, the number of independent DA's to be included in the OPE is much larger. These DA's are nevertheless well under control due to twist and conformal-spin expansion, and the resulting number of relevant input parameters is in fact not that large. The other complication is more serious because little is known about the background contributions of negative-parity baryons in the hadronic dispersion relations. We proposed a novel method to eliminate these "contamination" by combining sum rules obtained from different kinematical structures. As a by-product, our results are less sensitive to the particular choice of the interpolating currents. Furthermore, as a step forward with respect to the procedure used in [11] we worked out the double spectral density for the power suppressed contributions in the correlation functions where also the finite mass of the nucleon is taken into account. Our numerical results for $\Lambda_{c}\left(\Sigma_{c}\right) \rightarrow p$ and $\Lambda_{b} \rightarrow p$ form factors and $\Lambda_{c}\left(\Sigma_{c}\right) N D^{(*)}$ strong couplings provide a nontrivial test of self-consistency of different sum rules, and also of the reliability of our procedure of eliminating the negative parity states.

We also obtained the predictions for semileptonic and nonleptonic $\Lambda_{b}$ decays using the LCSR form factors. In particular, the partially integrated width of $\Lambda_{b} \rightarrow p \ell \nu_{l}$ can be used for the $\left|V_{u b}\right|$ determination from the future data on this decay. Furthermore, in a forthcoming publication [30] we will employ the LCSR results for the strong couplings of charmed baryons to estimate their production in proton-antiproton collisions in the PANDA energy region.

Future improvements of the nucleon DA's, and the calculation of gluon radiative corrections to the correlation functions will make the sum rule results obtained here more accurate. A considerable step forward will be to work out and incorporate in LCSR the $\Lambda$-baryon DA's. This will make possible an accurate calculation of the form factors needed in the FCNC decays such as $\Lambda_{b} \rightarrow \Lambda \gamma$ and $\Lambda_{b} \rightarrow \Lambda \ell^{+} \ell^{-}$. Finally, a very important task is to confront the LCSR predictions with the relations for the heavy baryon form factors and couplings derived from heavy-quark symmetries. In this paper we only made one step in this direction, by comparing the LCSR predictions with the relations for the form factors and strong couplings which follow from the heavy-quark spin symmetry. A more elaborated study including the quantitative analysis of HQET relations for the baryonic form factors based on LCSR calculations will be presented elsewhere. 


\section{Acknowledgements}

This work is supported by the German research foundation DFG under contract MA1187/101 and by the German Ministry of Research (BMBF), contract O6SI9192.

\section{A. Nucleon Distribution Amplitudes}

The nucleon $\rightarrow$ vacuum matrix element of a three-quark operator with light-like separations $\left(z^{2} \rightarrow 0\right)$ has the following decomposition [5, 9]:

$$
\begin{aligned}
& 4\left\langle 0\left|\epsilon^{i j k} u_{\alpha}^{i}\left(a_{1} z\right) u_{\beta}^{j}\left(a_{2} z\right) d_{\gamma}^{k}\left(a_{3} z\right)\right| N(P)\right\rangle \\
&=\mathcal{S}_{1} m_{N} C_{\alpha \beta}\left(\gamma_{5} u_{N}\right)_{\gamma}+\mathcal{S}_{2} m_{N}^{2} C_{\alpha \beta}\left(\not \gamma_{5} u_{N}\right)_{\gamma}+\mathcal{P}_{1} m_{N}\left(\gamma_{5} C\right)_{\alpha \beta}\left(u_{N}\right)_{\gamma} \\
&+\mathcal{P}_{2} m_{N}^{2}\left(\gamma_{5} C\right)_{\alpha \beta}\left(\not u_{N}\right)_{\gamma}+\left(\mathcal{V}_{1}+\frac{z^{2} m_{N}^{2}}{4} \mathcal{V}_{1}^{M}\right)(P C)_{\alpha \beta}\left(\gamma_{5} u_{N}\right)_{\gamma} \\
&+\mathcal{V}_{2} m_{N}(P C)_{\alpha \beta}\left(\not \gamma_{5} u_{N}\right)_{\gamma}+\mathcal{V}_{3} m_{N}\left(\gamma_{\mu} C\right)_{\alpha \beta}\left(\gamma^{\mu} \gamma_{5} u_{N}\right)_{\gamma} \\
&+\mathcal{V}_{4} m_{N}^{2}(\not C)_{\alpha \beta}\left(\gamma_{5} u_{N}\right)_{\gamma}+\mathcal{V}_{5} m_{N}^{2}\left(\gamma_{\mu} C\right)_{\alpha \beta}\left(i \sigma^{\mu \nu} z_{\nu} \gamma_{5} u_{N}\right)_{\gamma} \\
&+\mathcal{V}_{6} m_{N}^{3}(\not C)_{\alpha \beta}\left(\not \gamma \gamma_{5} u_{N}\right)_{\gamma}+\left(\mathcal{A}_{1}+\frac{z^{2} m_{N}^{2}}{4} \mathcal{A}_{1}^{M}\right)\left(\not \gamma_{5} C\right)_{\alpha \beta}\left(u_{N}\right)_{\gamma} \\
&+\mathcal{A}_{2} m_{N}\left(\not P \gamma_{5} C\right)_{\alpha \beta}\left(\not u_{N}\right)_{\gamma}+\mathcal{A}_{3} m_{N}\left(\gamma_{\mu} \gamma_{5} C\right)_{\alpha \beta}\left(\gamma^{\mu} u_{N}\right)_{\gamma} \\
&+\mathcal{A}_{4} m_{N}^{2}\left(\not \gamma_{5} C\right)_{\alpha \beta}\left(u_{N}\right)_{\gamma}+\mathcal{A}_{5} m_{N}^{2}\left(\gamma_{\mu} \gamma_{5} C\right)_{\alpha \beta}\left(i \sigma^{\mu \nu} z_{\nu} u_{N}\right)_{\gamma} \\
&+\mathcal{A}_{6} m_{N}^{3}\left(\not \gamma_{5} C\right)_{\alpha \beta}\left(\not u_{N}\right)_{\gamma}+\left(\mathcal{T}_{1}+\frac{z^{2} m_{N}^{2}}{4} \mathcal{T}_{1}^{M}\right)\left(P^{\nu} i \sigma_{\mu \nu} C\right)_{\alpha \beta}\left(\gamma^{\mu} \gamma_{5} u_{N}\right)_{\gamma} \\
&+\mathcal{T}_{2} m_{N}\left(z^{\mu} P^{\nu} i \sigma_{\mu \nu} C\right)_{\alpha \beta}\left(\gamma_{5} u_{N}\right)_{\gamma}+\mathcal{T}_{3} m_{N}\left(\sigma_{\mu \nu} C\right)_{\alpha \beta}\left(\sigma^{\mu \nu} \gamma_{5} u_{N}\right)_{\gamma} \\
&+\mathcal{T}_{4} m_{N}\left(P^{\nu} \sigma_{\mu \nu} C\right)_{\alpha \beta}\left(\sigma^{\mu \rho} z_{\rho} \gamma_{5} u_{N}\right)_{\gamma}+\mathcal{T}_{5} m_{N}^{2}\left(z^{\nu} i \sigma_{\mu \nu} C\right)_{\alpha \beta}\left(\gamma^{\mu} \gamma_{5} u_{N}\right)_{\gamma} \\
&+\mathcal{T}_{6} m_{N}^{2}\left(z^{\mu} P^{\nu} i \sigma_{\mu \nu} C\right)_{\alpha \beta}\left(\not \gamma_{5} u_{N}\right)_{\gamma}+\mathcal{T}_{7} m_{N}^{2}\left(\sigma_{\mu \nu} C\right)_{\alpha \beta}\left(\sigma^{\mu \nu} \not \gamma_{5} u_{N}\right)_{\gamma} \\
&+\mathcal{T}_{8} m_{N}^{3}\left(z^{\nu} \sigma_{\mu \nu} C\right)_{\alpha \beta}\left(\sigma^{\mu \rho} z_{\rho} \gamma_{5} u_{N}\right)_{\gamma},
\end{aligned}
$$

including the $O\left(z^{2}\right)$ corrections to the lowest twist-3 part of this decomposition. In the above, the gauge links maintaining gauge invariance are not shown for brevity. The calligraphic coefficients $\mathcal{S}_{1,2}, \mathcal{P}_{1,2}, \mathcal{V}_{1, \ldots, 6}, \mathcal{A}_{1, \ldots, 6}, \mathcal{T}_{1, \ldots, 8}, \mathcal{V}_{1}^{M}, \mathcal{A}_{1}^{M}, \mathcal{T}_{1}^{M}$ at different Dirac structures, are related to the integrals containing the nucleon DA's depending on the longitudinal momentum fractions $x_{i}$. The general relation reads

$$
\mathcal{F}\left(a_{1}, a_{2}, a_{3},(P \cdot z)\right)=\int d x_{1} d x_{2} d x_{3} \delta\left(1-x_{1}-x_{2}-x_{3}\right) e^{-i(P \cdot z) \sum_{i} x_{i} a_{i}} F\left(x_{i}\right),
$$

where our choice of the three-quark configuration on the light-cone corresponds to $a_{1,3}=$ $0, a_{2}=1$. The function $\mathcal{F}$ and the integrand on r.h.s. for all coefficients in (71) are collected in Table 4. The resulting decomposition contains altogether 27 DA's. We use their expressions obtained in [6, 9] to the next-to-leading order in the conformal spin expansion. The three twist-3 DA's $V_{1}, A_{1}, T_{1}$ are presented in eq. (25) and the others are: 


\begin{tabular}{|c|c||c|c|}
\hline \hline $\mathcal{F}$ & integrand on r.h.s. of (72) & $\mathcal{F}$ & integrand on r.h.s. of (72) \\
\hline $\mathcal{S}_{1}$ & $S_{1}$ & $2(P \cdot z) \mathcal{S}_{2}$ & $S_{1}-S_{2}$ \\
\hline $\mathcal{P}_{1}$ & $P_{1}$ & $2(P \cdot z) \mathcal{P}_{2}$ & $P_{2}-P_{1}$ \\
\hline $\mathcal{V}_{1}$ & $V_{1}$ & $2(P \cdot z) \mathcal{V}_{2}$ & $V_{1}-V_{2}-V_{3}$ \\
\hline $2 \mathcal{V}_{3}$ & $V_{3}$ & $4(P \cdot z) \mathcal{V}_{4}$ & $-2 V_{1}+V_{3}+V_{4}+2 V_{5}$ \\
\hline $4(P \cdot z) \mathcal{V}_{5}$ & $V_{4}-V_{3}$ & $4(P \cdot z)^{2} \mathcal{V}_{6}$ & $-V_{1}+V_{2}+V_{3}+V_{4}+V_{5}-V_{6}$ \\
\hline $\mathcal{A}_{1}$ & $A_{1}$ & $2(P \cdot z) \mathcal{A}_{2}$ & $-A_{1}+A_{2}-A_{3}$ \\
\hline $2 \mathcal{A}_{3}$ & $A_{3}$ & $4(P \cdot z) \mathcal{A}_{4}$ & $-2 A_{1}-A_{3}-A_{4}+2 A_{5}$ \\
\hline $4(P \cdot z) \mathcal{A}_{5}$ & $A_{3}-A_{4}$ & $4(P \cdot z)^{2} \mathcal{A}_{6}$ & $A_{1}-A_{2}+A_{3}+A_{4}-A_{5}+A_{6}$ \\
\hline $\mathcal{T}_{1}$ & $T_{1}$ & $2(P \cdot z) \mathcal{T}_{2}$ & $T_{1}+T_{2}-2 T_{3}$ \\
\hline $2 \mathcal{T}_{3}$ & $T_{7}$ & $2(P \cdot z) \mathcal{T}_{4}$ & $T_{1}-T_{2}-2 T_{7}$ \\
\hline $2(P \cdot z) \mathcal{T}_{5}$ & $-T_{1}+T_{5}+2 T_{8}$ & $4(P \cdot z)^{2} \mathcal{T}_{6}$ & $2 T_{2}-2 T_{3}-2 T_{4}+2 T_{5}+2 T_{7}+2 T_{8}$ \\
\hline $4(P \cdot z) \mathcal{T}_{7}$ & $T_{7}-T_{8}$ & $4(P \cdot z)^{2} \mathcal{T}_{8}$ & $-T_{1}+T_{2}+T_{5}-T_{6}+2 T_{7}+2 T_{8}$ \\
\hline $\mathcal{V}_{1}^{M}$ & $V_{1}^{M}$ & $\mathcal{A}_{1}^{M}$ & $A_{1}^{M}$ \\
\hline $\mathcal{T}_{1}^{M}$ & $T_{1}^{M}$ & & \\
\hline \hline
\end{tabular}

Table 4: Terms of the decomposition (171) and their relation to the nucleon DA's via Eq.(72).

- twist-4 DA's:

$$
\begin{array}{r}
V_{2}\left(x_{i}\right)=24 x_{1} x_{2}\left[\phi_{4}^{0}+\phi_{4}^{+}\left(1-5 x_{3}\right)\right], \quad A_{2}\left(x_{i}\right)=24 x_{1} x_{2}\left(x_{2}-x_{1}\right) \phi_{4}^{-}, \\
T_{2}\left(x_{i}\right)=24 x_{1} x_{2}\left[\xi_{4}^{0}+\xi_{4}^{+}\left(1-5 x_{3}\right)\right], \\
V_{3}\left(x_{i}\right)=12 x_{3}\left[\psi_{4}^{0}\left(1-x_{3}\right)+\psi_{4}^{+}\left(1-x_{3}-10 x_{1} x_{2}\right)+\psi_{4}^{-}\left(x_{1}^{2}+x_{2}^{2}-x_{3}\left(1-x_{3}\right)\right)\right], \\
A_{3}\left(x_{i}\right)=12 x_{3}\left(x_{2}-x_{1}\right)\left[\left(\psi_{4}^{0}+\psi_{4}^{+}\right)+\psi_{4}^{-}\left(1-2 x_{3}\right)\right], \\
\\
T_{3}\left(x_{i}\right)=6 x_{3}\left[\left(\phi_{4}^{0}+\psi_{4}^{0}+\xi_{4}^{0}\right)\left(1-x_{3}\right)+\left(\phi_{4}^{+}+\psi_{4}^{+}+\xi_{4}^{+}\right)\left(1-x_{3}-10 x_{1} x_{2}\right)\right. \\
\left.+\left(\phi_{4}^{-}-\psi_{4}^{-}+\xi_{4}^{-}\right)\left(x_{1}^{2}+x_{2}^{2}-x_{3}\left(1-x_{3}\right)\right)\right], \\
T_{7}\left(x_{i}\right)=6 x_{3}\left[\left(\phi_{4}^{0}+\psi_{4}^{0}-\xi_{4}^{0}\right)\left(1-x_{3}\right)+\left(\phi_{4}^{+}+\psi_{4}^{+}-\xi_{4}^{+}\right)\left(1-x_{3}-10 x_{1} x_{2}\right)\right] \\
\left.+\left(\phi_{4}^{-}-\psi_{4}^{-}-\xi_{4}^{-}\right)\left(x_{1}^{2}+x_{2}^{2}-x_{3}\left(1-x_{3}\right)\right)\right], \\
S_{1}\left(x_{i}\right)=6 x_{3}\left(x_{2}-x_{1}\right)\left[\left(\phi_{4}^{0}+\psi_{4}^{0}+\xi_{4}^{0}+\phi_{4}^{+}+\psi_{4}^{+}+\xi_{4}^{+}\right)+\left(\phi_{4}^{-}-\psi_{4}^{-}+\xi_{4}^{-}\right)\left(1-2 x_{3}\right)\right], \\
P_{1}\left(x_{i}\right)=6 x_{3}\left(x_{1}-x_{2}\right)\left[\left(\phi_{4}^{0}+\psi_{4}^{0}-\xi_{4}^{0}+\phi_{4}^{+}+\psi_{4}^{+}-\xi_{4}^{+}\right)+\left(\phi_{4}^{-}-\psi_{4}^{-}-\xi_{4}^{-}\right)\left(1-2 x_{3}\right)\right],
\end{array}
$$

- twist-5 DA's:

$$
\begin{array}{r}
V_{4}\left(x_{i}\right)=3\left[\psi_{5}^{0}\left(1-x_{3}\right)+\psi_{5}^{+}\left(1-x_{3}-2\left(x_{1}^{2}+x_{2}^{2}\right)\right)+\psi_{5}^{-}\left(2 x_{1} x_{2}-x_{3}\left(1-x_{3}\right)\right)\right], \\
A_{4}\left(x_{i}\right)=3\left(x_{2}-x_{1}\right)\left[-\psi_{5}^{0}+\psi_{5}^{+}\left(1-2 x_{3}\right)+\psi_{5}^{-} x_{3}\right],
\end{array}
$$




$$
\begin{gathered}
T_{4}\left(x_{i}\right)=\frac{3}{2}\left[\left(\phi_{5}^{0}+\psi_{5}^{0}+\xi_{5}^{0}\right)\left(1-x_{3}\right)+\left(\phi_{5}^{+}+\psi_{5}^{+}+\xi_{5}^{+}\right)\left(1-x_{3}-2\left(x_{1}^{2}+x_{2}^{2}\right)\right)\right] \\
+\left(\phi_{5}^{-}-\psi_{5}^{-}+\xi_{5}^{-}\right)\left(2 x_{1} x_{2}-x_{3}\left(1-x_{3}\right)\right), \\
T_{8}\left(x_{i}\right)=\frac{3}{2}\left[\left(\phi_{5}^{0}+\psi_{5}^{0}-\xi_{5}^{0}\right)\left(1-x_{3}\right)+\left(\phi_{5}^{+}+\psi_{5}^{+}-\xi_{5}^{+}\right)\left(1-x_{3}-2\left(x_{1}^{2}+x_{2}^{2}\right)\right)\right. \\
\left.+\left(\phi_{5}^{-}-\psi_{5}^{-}+\xi_{5}^{-}\right)\left(2 x_{1} x_{2}-x_{3}\left(1-x_{3}\right)\right)\right], \\
V_{5}\left(x_{i}\right)=6 x_{3}\left[\phi_{5}^{0}+\phi_{5}^{+}\left(1-2 x_{3}\right)\right], \quad A_{5}\left(x_{i}\right)=6 x_{3}\left(x_{2}-x_{1}\right) \phi_{5}^{-}, \\
T_{5}\left(x_{i}\right)=6 x_{3}\left[\xi_{5}^{0}+\xi_{5}^{+}\left(1-2 x_{3}\right)\right] \\
S_{2}\left(x_{i}\right)=\frac{3}{2}\left(x_{2}-x_{1}\right)\left[-\left(\phi_{5}^{0}+\psi_{5}^{0}+\xi_{5}^{0}\right)+\left(\phi_{5}^{+}+\psi_{5}^{+}+\xi_{5}^{+}\right)\left(1-2 x_{3}\right)\right. \\
\left.+\left(\phi_{5}^{-}-\psi_{5}^{-}+\xi_{5}^{-}\right) x_{3}\right] \\
P_{2}\left(x_{i}\right)=\frac{3}{2}\left(x_{1}-x_{2}\right)\left[-\left(\phi_{5}^{0}+\psi_{5}^{0}-\xi_{5}^{0}\right)+\left(\phi_{5}^{+}+\psi_{5}^{+}-\xi_{5}^{+}\right)\left(1-2 x_{3}\right)\right. \\
\left.+\left(\phi_{5}^{-}-\psi_{5}^{-}-\xi_{5}^{-}\right) x_{3}\right],
\end{gathered}
$$

- twist-6 DA's:

$$
\begin{array}{r}
V_{6}\left(x_{i}\right)=2\left[\phi_{6}^{0}+\phi_{6}^{+}\left(1-3 x_{3}\right)\right], \quad A_{6}\left(x_{i}\right)=2\left(x_{2}-x_{1}\right) \phi_{6}^{-} \\
T_{6}\left(x_{i}\right)=2\left[\phi_{6}^{0}-\frac{1}{2}\left(\phi_{6}^{+}-\phi_{6}^{-}\right)\left(1-3 x_{3}\right) .\right.
\end{array}
$$

The expressions for the remaining three DA's $\left(V_{1}^{M}, A_{1}^{M}, T_{1}^{M}\right)$ determining the $z^{2}$ corrections are shown below.

Furthermore, following Ref. [8], shorthand notations for the combinations of nucleon DA's are used:

$$
\begin{aligned}
S_{12}=S_{1}-S_{2}, & P_{21}=P_{2}-P_{1}, \\
V_{1345}=-2 V_{1}+V_{3}+V_{4}+2 V_{5}, & V_{43}=V_{4}-V_{3}, \\
V_{123456}=-V_{1}+V_{2}+V_{3}+V_{4}+V_{5}-V_{6}, & V_{123}=V_{1}-V_{2}-V_{3} \\
A_{1345}=-2 A_{1}-A_{3}-A_{4}+2 A_{5}, & A_{34}=A_{3}-A_{4} \\
A_{123456}=A_{1}-A_{2}+A_{3}+A_{4}-A_{5}+A_{6}, & A_{123}=-A_{1}+A_{2}-A_{3} \\
T_{78}=T_{7}-T_{8}, & T_{123}=T_{1}+T_{2}-2 T_{3}, \\
T_{234578=2 T_{2}-2 T_{3}-2 T_{4}+2 T_{5}+2 T_{7}+2 T_{8},} & T_{127}=T_{1}-T_{2}-2 T_{7}, \\
T_{125678}=-T_{1}+T_{2}+T_{5}-T_{6}+2 T_{7}+2 T_{8}, & T_{158}=-T_{1}+T_{5}+2 T_{8} .
\end{aligned}
$$


In addition, the following notations are introduced for the integrals:

$$
\begin{aligned}
& \tilde{F}\left(x_{2}\right)=\int_{0}^{1-x_{2}} d x_{1} F\left(x_{1}, x_{2}, 1-x_{1}-x_{2}\right), \\
& \tilde{\tilde{F}}\left(x_{2}\right)=\int_{1}^{x_{2}} d x_{2}^{\prime} \int_{0}^{1-x_{2}^{\prime}} d x_{1} F\left(x_{1}, x_{2}^{\prime}, 1-x_{1}-x_{2}^{\prime}\right), \\
& \tilde{\tilde{F}}\left(x_{2}\right)=\int_{1}^{x_{2}} d x_{2}^{\prime} \int_{1}^{x_{2}^{\prime}} d x_{2}^{\prime \prime} \int_{0}^{1-x_{2}^{\prime \prime}} d x_{1} F\left(x_{1}, x_{2}^{\prime \prime}, 1-x_{1}-x_{2}^{\prime \prime}\right)
\end{aligned}
$$

where $F$ is one of the DA's.

Finally, the DA's originating from $z^{2}$ corrections enter in the integrated form denoted as $\tilde{V}_{1}^{M}\left(x_{2}\right), \tilde{A}_{1}^{M}\left(x_{2}\right)$ and $\tilde{T}_{1}^{M}\left(x_{2}\right)$, where

$$
\begin{aligned}
\tilde{V}_{1}^{M}\left(x_{2}\right) & =\frac{x_{2}^{2}}{24}\left[f_{N} C_{f}^{u}\left(x_{2}\right)+\lambda_{1} C_{\lambda}^{u}\left(x_{2}\right)\right], \\
\tilde{A}_{1}^{M}\left(x_{2}\right) & =\frac{x_{2}^{2}}{24}\left(1-x_{2}\right)^{3}\left[f_{N} D_{f}^{u}\left(x_{2}\right)+\lambda_{1} D_{\lambda}^{u}\left(x_{2}\right)\right], \\
\tilde{T}_{1}^{M}\left(x_{2}\right) & =\frac{x_{2}^{2}}{48}\left[f_{N} E_{f}^{u}\left(x_{2}\right)+\lambda_{1} E_{\lambda}^{u}\left(x_{2}\right)\right],
\end{aligned}
$$

with

$$
\begin{aligned}
& C_{f}^{u}\left(x_{2}\right)=\left(1-x_{2}\right)^{3}\left[113+495 x_{2}-552 x_{2}^{2}-10 A_{1}^{u}\left(1-3 x_{2}\right)\right. \\
&\left.+2 V_{1}^{d}\left(113-951 x_{2}+828 x_{2}^{2}\right)\right] \\
& C_{\lambda}^{u}\left(x_{2}\right)=\left.-\left(1-x_{2}\right)^{3}\left[13-20 f_{1}^{d}+3 x_{2}+10 f_{1}^{u}\left(1-3 x_{2}\right)\right)\right] \\
& D_{f}^{u}\left(x_{2}\right)=11+45 x_{2}-2 A_{1}^{u}\left(113-951 x_{2}+828 x_{2}^{2}\right)+10 V_{1}^{d}\left(1-30 x_{2}\right), \\
& D_{\lambda}^{u}\left(x_{2}\right)=29-45 x_{2}-10 f_{1}^{u}\left(7-9 x_{2}\right)-20 f_{1}^{d}\left(5-6 x_{2}\right), \\
& E_{f}^{u}\left(x_{2}\right)=\quad-\left[( 1 - x _ { 2 } ) \left(3\left(439+71 x_{2}-621 x_{2}^{2}+587 x_{2}^{3}-184 x_{2}^{4}\right)\right.\right. \\
& \quad+4 A_{1}^{u}\left(1-x_{2}\right)^{2}\left(59-483 x_{2}+414 x_{2}^{2}\right) \\
&\left.\left.\quad-4 V_{1}^{d}\left(1301-619 x_{2}-769 x_{2}^{2}+1161 x_{2}^{3}-414 x_{2}^{4}\right)\right)\right] \\
& \quad-12\left(73-220 V_{1}^{d}\right) \ln x_{2}, \\
& E_{\lambda}^{u}\left(x_{2}\right)=\quad-\left[( 1 - x _ { 2 } ) \left(5-211 x_{2}+281 x_{2}^{2}-111 x_{2}^{3}\right.\right. \\
& \quad+10\left(1+61 x_{2}-83 x_{2}^{2}+33 x_{2}^{3}\right) f_{1}^{d} \\
&\left.\left.\quad-40\left(1-x_{2}\right)^{2}\left(2-3 x_{2}\right) f_{1}^{u}\right)\right]-12\left(3-10 f_{1}^{d}\right) \ln x_{2} .
\end{aligned}
$$

The terms proportional to $\ln x_{2}$ in the above DA $\tilde{T}_{1}^{M}\left(x_{2}\right)$ are the only non-polynomial ones in the whole OPE expressions. In the case of LCSR for the form factors their transformation to a dispersion form is straightforward. However, for the strong coupling sum rules a double dispersion form of such nonpolynomial terms demands a separate derivation. In fact, these terms turn out to have negligible coefficients with our choice of the DA parameters $V_{1}^{d}$ and $f_{1}^{d}$ and hence are simply neglected. 
The coefficients $\phi_{i}^{( \pm, 0)}, \psi_{i}^{( \pm, 0)}$ and $\xi_{i}^{( \pm, 0)}(i=3,4,5,6)$ determining the normalization and shape of DA's can be expressed through the eight independent parameters listed in (58) and (59). The corresponding relations for the leading conformal spin in DA's are

$$
\begin{array}{rlrl}
\phi_{3}^{0} & =\phi_{6}^{0}=f_{N}, & \phi_{4}^{0}=\phi_{4}^{0}=\frac{1}{2}\left(f_{N}+\lambda_{1}\right), \\
\xi_{4}^{0}=\xi_{5}^{0}=\frac{1}{6} \lambda_{2}, & \psi_{4}^{0}=\psi_{5}^{0}=\frac{1}{2}\left(f_{N}-\lambda_{1}\right) .
\end{array}
$$

For the next-to-leading conformal spin,

- in twist-3 DA's:

$$
\phi_{3}^{-}=\frac{21}{2} f_{N} A_{1}^{u}, \quad \phi_{3}^{+}=\frac{7}{2} f_{N}\left(1-3 V_{1}^{d}\right),
$$

- in twist-4 DA's:

$$
\begin{aligned}
\phi_{4}^{+} & =\frac{1}{4}\left[f_{N}\left(3-10 V_{1}^{d}\right)+\lambda_{1}\left(3-10 f_{1}^{d}\right)\right], \\
\phi_{4}^{-} & =-\frac{5}{4}\left[f_{N}\left(1-2 A_{1}^{u}\right)-\lambda_{1}\left(1-2 f_{1}^{d}-4 f_{1}^{u}\right)\right], \\
\psi_{4}^{+} & =-\frac{1}{4}\left[f_{N}\left(2+5 A_{1}^{u}-5 V_{1}^{d}\right)-\lambda_{1}\left(2-5 f_{1}^{d}-5 f_{1}^{u}\right)\right] \\
\psi_{4}^{-} & =\frac{5}{4}\left[f_{N}\left(2-A_{1}^{u}-3 V_{1}^{d}\right)-\lambda_{1}\left(2-7 f_{1}^{d}+f_{1}^{u}\right)\right] \\
\xi_{4}^{+} & =\frac{1}{16} \lambda_{2}\left(4-15 f_{2}^{d}\right), \quad \xi_{4}^{-}=\frac{5}{16} \lambda_{2}\left(4-15 f_{2}^{d}\right),
\end{aligned}
$$

- in twist-5 DA's:

$$
\begin{aligned}
\phi_{5}^{+} & =-\frac{5}{6}\left[f_{N}\left(3+4 V_{1}^{d}\right)-\lambda_{1}\left(1-4 f_{1}^{d}\right)\right], \\
\phi_{5}^{-} & =-\frac{5}{3}\left[f_{N}\left(1-2 A_{1}^{u}\right)-\lambda_{1}\left(f_{1}^{d}-f_{1}^{u}\right)\right], \\
\psi_{5}^{+} & =-\frac{5}{6}\left[f_{N}\left(5+2 A_{1}^{u}-2 V_{1}^{d}\right)-\lambda_{1}\left(1-2 f_{1}^{d}-2 f_{1}^{u}\right)\right], \\
\psi_{5}^{-} & =\frac{5}{3}\left[f_{N}\left(2-A_{1}^{u}-3 V_{1}^{d}\right)+\lambda_{1}\left(f_{1}^{d}-f_{1}^{u}\right)\right], \\
\xi_{5}^{+} & =\frac{5}{36} \lambda_{2}\left(2-9 f_{2}^{d}\right), \quad \xi_{5}^{-}=-\frac{5}{4} \lambda_{2} f_{2}^{d},
\end{aligned}
$$

- in twist-6 DA's:

$$
\begin{aligned}
\phi_{6}^{+} & =\frac{1}{2}\left[f_{N}\left(1-4 V_{1}^{d}\right)-\lambda_{1}\left(1-2 f_{1}^{d}\right)\right] \\
\phi_{6}^{-} & =\frac{1}{2}\left[f_{N}\left(1+4 A_{1}^{u}\right)+\lambda_{1}\left(1-4 f_{1}^{d}-2 f_{1}^{u}\right)\right]
\end{aligned}
$$




\section{B. Correlation function in the LCSR for $\Lambda_{c} \rightarrow N$ form factors}

\section{B.1. pseudoscalar transition current}

The invariant amplitudes $\Pi_{j}^{(i)}\left((P-q)^{2}, q^{2}\right)$ of the correlation function with the pseudoscalar transition current $j_{5}$ are given in (26) $)$, where the coefficient functions $\tilde{\omega}_{j n}^{(i)}$ with $i=\mathcal{P}, \mathcal{A}, j=1,2$ and $n=1,2,3$, (after replacement of $(P-q)^{2}$ described in Sect. 4) are listed below for:

- the pseudoscalar interpolating current

$$
\begin{aligned}
\omega_{11}^{(\mathcal{P})} & =\frac{m_{N}}{2}\left[\left(m_{c}-x m_{N}\right) \Phi_{1}^{(\mathcal{P})}-m_{N} \Phi_{2}^{(\mathcal{P})}\right] \\
\omega_{12}^{(\mathcal{P})} & =-\frac{m_{N}^{2}}{2}\left[m_{c}\left(m_{c}-x m_{N}\right) \Phi_{2}^{(\mathcal{P})}+2 x m_{N}^{2} \Phi_{3}^{(\mathcal{P})}\right], \\
\omega_{13}^{(\mathcal{P})} & =2 m_{N}^{3} m_{c}^{2}\left(m_{c}-x m_{N}\right) \Phi_{3}^{(\mathcal{P})} \\
\omega_{21}^{(\mathcal{P})} & =-\frac{m_{N}}{2} \Phi_{1}^{(\mathcal{P})}, \quad \omega_{22}^{(\mathcal{P})}=\frac{m_{N}^{2}}{2}\left(m_{c} \Phi_{2}^{(\mathcal{P})}-2 m_{N} \Phi_{3}^{(\mathcal{P})}\right), \\
\omega_{23}^{(\mathcal{P})} & =-2 m_{N}^{3} m_{c}^{2} \Phi_{3}^{(\mathcal{P})},
\end{aligned}
$$

where the functions $\Phi_{i}^{(\mathcal{P})}$ in the above equations are

$$
\begin{aligned}
& \Phi_{1}^{(\mathcal{P})}=2 \tilde{A}_{1}+4 \tilde{A}_{3}+2 \tilde{A}_{123}+2 \tilde{P}_{1}+2 \tilde{S}_{1}+6 \tilde{T}_{1}-12 \tilde{T}_{7}-\tilde{T}_{123}-5 \tilde{T}_{127} \\
& -2 \tilde{V}_{1}+4 \tilde{V}_{3}+2 \tilde{V}_{123} \\
& \Phi_{2}^{(\mathcal{P})}=3 \tilde{\tilde{A}}_{34}+2 \tilde{\tilde{A}}_{123}-\tilde{\tilde{A}}_{1345}-2 \tilde{\tilde{P}}_{21}+2 \tilde{\tilde{S}}_{12}-12 \tilde{\tilde{T}}_{78}-2 \tilde{\tilde{T}}_{123}-4 \tilde{\tilde{T}}_{127} \\
& -6 \tilde{\tilde{T}}_{158}+\tilde{\tilde{T}}_{234578}-3 \tilde{\tilde{V}}_{43}+2 \tilde{\tilde{V}}_{123}+\tilde{\tilde{V}}_{1345}, \\
& \Phi_{3}^{(\mathcal{P})}=-\tilde{A}_{1}^{M}-3 \tilde{T}_{1}^{M}+\tilde{V}_{1}^{M}+\tilde{\tilde{\tilde{A}}}_{123456}-3 \tilde{\tilde{\tilde{T}}}_{125678}+\tilde{\tilde{\tilde{T}}}_{234578}+\tilde{\tilde{\tilde{V}}}_{123456} ;
\end{aligned}
$$

- the axial-vector interpolating current

$$
\begin{aligned}
\omega_{11}^{(\mathcal{A})}= & 2 \frac{m_{c}^{2}-q^{2}}{x} \Phi_{1}^{(\mathcal{A})}+x m_{N}^{2}\left[2 \Phi_{1}^{(\mathcal{A})}+\Phi_{2}^{(\mathcal{A})}\right]+m_{N} m_{c} \Phi_{3}^{(\mathcal{A})}+m_{N}^{2}\left[\Phi_{4}^{(\mathcal{A})}+\frac{2 \Phi_{5}^{(\mathcal{A})}}{x}\right] \\
\omega_{12}^{(\mathcal{A})}= & -m_{N}^{2}\left[2\left(x^{2} m_{N}^{2}-q^{2}\right) \Phi_{6}^{(\mathcal{A})}+x m_{N} m_{c} \Phi_{7}^{(\mathcal{A})}+m_{c}^{2} \Phi_{8}^{(\mathcal{A})}-2 \frac{q^{2}+m_{c}^{2}}{x} \Phi_{5}^{(\mathcal{A})}\right. \\
& \left.+2 x m_{N}^{2} \Phi_{9}^{(\mathcal{A})}\right] \\
\omega_{13}^{(\mathcal{A})}= & 4 \frac{m_{N}^{2}}{x}\left[m_{c}^{2}\left(q^{2}-m_{c}^{2}\right) \Phi_{5}^{(\mathcal{A})}-x^{2} m_{N}^{2} m_{c}^{2} \Phi_{9}^{(\mathcal{A})}+x m_{N} m_{c}^{3} \Phi_{10}^{(\mathcal{A})}\right]
\end{aligned}
$$




$$
\begin{aligned}
\omega_{21}^{(\mathcal{A})} & =\frac{m_{N}}{x}\left[2 \Phi_{6}^{(\mathcal{A})}+x \Phi_{2}^{(\mathcal{A})}\right], \quad \omega_{23}^{(\mathcal{A})}=4 m_{N}^{3} m_{c}^{2} \Phi_{11}^{(\mathcal{A})} \\
\omega_{22}^{(\mathcal{A})} & =\frac{m_{N}}{x}\left[2\left(q^{2}-m_{c}^{2}-x^{2} m_{N}^{2}\right) \Phi_{6}^{(\mathcal{A})}-x m_{N} m_{c} \Phi_{7}^{(\mathcal{A})}+2 x m_{N}^{2} \Phi_{11}^{(\mathcal{A})}\right]
\end{aligned}
$$

where the functions $\Phi_{i}^{(\mathcal{A})}$ are

$$
\begin{aligned}
& \Phi_{1}^{(\mathcal{A})}=\tilde{A}_{1}+2 \tilde{T}_{1}+\tilde{V}_{1}, \\
& \Phi_{2}^{(\mathcal{A})}=2 \tilde{A}_{3}-2 \tilde{P}_{1}+2 \tilde{S}_{1}-2 \tilde{T}_{1}+\tilde{T}_{123}+\tilde{T}_{127}-2 \tilde{V}_{3}, \\
& \Phi_{3}^{(\mathcal{A})}=2 \tilde{A}_{1}+4 \tilde{A}_{3}+2 \tilde{A}_{123}-4 \tilde{P}_{1}+4 \tilde{S}_{1}+2 \tilde{V}_{1}-4 \tilde{V}_{3}-2 \tilde{V}_{123}, \\
& \Phi_{4}^{(\mathcal{A})}=-2 \tilde{\tilde{A}}_{1345}+2 \tilde{\tilde{P}}_{21}+2 \tilde{\tilde{S}}_{12}-2 \tilde{\tilde{T}}_{123}+4 \tilde{\tilde{T}}_{127}-6 \tilde{\tilde{T}}_{158}+3 \tilde{\tilde{T}}_{234578}-2 \tilde{\tilde{V}}_{1345}, \\
& \Phi_{5}^{(\mathcal{A})}=\tilde{A}_{1}^{M}+2 \tilde{T}_{1}^{M}+\tilde{V}_{1}^{M}-\tilde{\tilde{T}}_{234578}, \\
& \Phi_{6}^{(\mathcal{A})}=\tilde{\tilde{A}}_{123}-\tilde{\tilde{T}}_{123}-\tilde{\tilde{T}}_{127}-\tilde{\tilde{V}}_{123}, \\
& \Phi_{7}^{(\mathcal{A})}=-3 \tilde{\tilde{A}}_{34}-2 \tilde{\tilde{A}}_{123}+\tilde{\tilde{A}}_{1345}-4 \tilde{\tilde{P}}_{21}-4 \tilde{\tilde{S}}_{12}-3 \tilde{\tilde{V}}_{43}+2 \tilde{\tilde{V}}_{123}+\tilde{\tilde{V}}_{1345}, \\
& \Phi_{8}^{(\mathcal{A})}=2 \tilde{\tilde{A}}_{123}+2 \tilde{\tilde{A}}_{1345}-2 \tilde{\tilde{P}}_{21}-2 \tilde{\tilde{S}}_{12}-6 \tilde{\tilde{T}}_{127}+6 \tilde{\tilde{T}}_{158}-3 \tilde{\tilde{T}}_{234578}-2 \tilde{\tilde{V}}_{123}+2 \tilde{\tilde{V}}_{1345}, \\
& \Phi_{9}^{(\mathcal{A})}=\tilde{A}_{1}^{M}+\tilde{T}_{1}^{M}+\tilde{V}_{1}^{M}-2 \tilde{\tilde{\tilde{A}}}_{123456}+3 \tilde{\tilde{\tilde{T}}}_{125678}-2 \tilde{\tilde{\tilde{T}}}_{234578}+2 \tilde{\tilde{\tilde{V}}}_{123456}, \\
& \Phi_{10}^{(\mathcal{A})}=-\tilde{A}_{1}^{M}-\tilde{V}_{1}^{M}+\tilde{\tilde{\tilde{A}}}_{123456}-\tilde{\tilde{\tilde{V}}}_{123456}, \\
& \Phi_{11}^{(\mathcal{A})}=\tilde{T}_{1}^{M}+2 \tilde{\tilde{\tilde{A}}}_{123456}-3 \tilde{\tilde{\tilde{T}}}_{125678}+\tilde{\tilde{\tilde{T}}}_{234578}-2 \tilde{\tilde{\tilde{V}}}_{123456} .
\end{aligned}
$$

\section{B.2. vector transition current}

The invariant amplitudes $\tilde{\Pi}_{j}^{(i)}\left((P-q)^{2}, q^{2}\right)$ for the correlation function with the vector transition current $j_{\mu}$ are given by Eq. (26) with the replacement of the coefficient $m_{c} / 4 \rightarrow 1 / 4$. The coefficient functions $\tilde{\omega}_{j n}^{(i)}$ with $i=\mathcal{P}, \mathcal{A}, j=1,2, \ldots 6$ and $n=1,2,3$ are listed below for:

- the pseudoscalar interpolating current

$$
\begin{aligned}
\tilde{\omega}_{11}^{(\mathcal{P})} & =x m_{N} \tilde{\Phi}_{1}^{(\mathcal{P})}, \quad \tilde{\omega}_{12}^{(\mathcal{P})}=x m_{N}^{3}\left[x \tilde{\Phi}_{2}^{(\mathcal{P})}+2 \tilde{\Phi}_{3}^{(\mathcal{P})}\right], \\
\tilde{\omega}_{13}^{(\mathcal{P})} & =4 x m_{N}^{3} m_{c}^{2} \tilde{\Phi}_{3}^{(\mathcal{P})} \\
& \tilde{\omega}_{21}^{(\mathcal{P})}=\tilde{\omega}_{23}^{(\mathcal{P})}=0, \quad \tilde{\omega}_{22}^{(\mathcal{P})}=-x m_{N}^{2} \tilde{\Phi}_{2}^{(\mathcal{P})},
\end{aligned}
$$




$$
\begin{gathered}
\tilde{\omega}_{31}^{(\mathcal{P})}=\frac{m_{N}}{2}\left(m_{c}-x m_{N}\right) \tilde{\Phi}_{1}^{(\mathcal{P})} \\
\tilde{\omega}_{32}^{(\mathcal{P})}=-\frac{m_{N}^{2}}{2}\left[m_{c}\left(m_{c}-x m_{N}\right) \tilde{\Phi}_{2}^{(\mathcal{P})}+2 x m_{N}^{2} \tilde{\Phi}_{3}^{(\mathcal{P})}\right] \\
\tilde{\omega}_{33}^{(\mathcal{P})}=2 m_{N}^{3} m_{c}^{2}\left(m_{c}-x m_{N}\right) \tilde{\Phi}_{3}^{(\mathcal{P})} \\
\tilde{\omega}_{41}^{(\mathcal{P})}=\frac{m_{N}}{2} \tilde{\Phi}_{1}^{(\mathcal{P})}, \quad \tilde{\omega}_{42}^{(\mathcal{P})}=\frac{m_{N}^{2}}{2}\left[-m_{c} \tilde{\Phi}_{2}^{(\mathcal{P})}+2 m_{N} \tilde{\Phi}_{3}^{(\mathcal{P})}\right], \\
\tilde{\omega}_{43}^{(\mathcal{P})}=2 m_{N}^{3} m_{c}^{2} \tilde{\Phi}_{3}^{(\mathcal{P})}, \\
\tilde{\omega}_{51}^{(\mathcal{P})}=-m_{N} \tilde{\Phi}_{1}^{(\mathcal{P})}, \quad \tilde{\omega}_{52}^{(\mathcal{P})}=-m_{N}^{3}\left[x \tilde{\Phi}_{2}^{(\mathcal{P})}+2 \tilde{\Phi}_{3}^{(\mathcal{P})}\right] \\
\tilde{\omega}_{53}^{(\mathcal{P})}=-4 m_{N}^{3} m_{c}^{2} \tilde{\Phi}_{3}^{(\mathcal{P})}, \\
\tilde{\omega}_{61}^{(\mathcal{P})}=\tilde{\omega}_{63}^{(\mathcal{P})}=0, \quad \tilde{\omega}_{62}^{(\mathcal{P})}=m_{N}^{2} \tilde{\Phi}_{2}^{(\mathcal{P})},
\end{gathered}
$$

where the functions $\tilde{\Phi}_{i}^{(\mathcal{P})}$ are given by

$$
\begin{aligned}
& \tilde{\Phi}_{1}^{(\mathcal{P})}=2 \tilde{A}_{1}+4 \tilde{A}_{3}+2 \tilde{A}_{123}+2 \tilde{P}_{1}+2 \tilde{S}_{1}+6 \tilde{T}_{1}-12 \tilde{T}_{7}-\tilde{T}_{123}-5 \tilde{T}_{127} \\
& -2 \tilde{V}_{1}+4 \tilde{V}_{3}+2 \tilde{V}_{123} \\
& \tilde{\Phi}_{2}^{(\mathcal{P})}=3 \tilde{\tilde{A}}_{34}+2 \tilde{\tilde{A}}_{123}-\tilde{\tilde{A}}_{1345}-2 \tilde{\tilde{P}}_{21}+2 \tilde{\tilde{S}}_{12}-12 \tilde{\tilde{T}}_{78}-2 \tilde{\tilde{T}}_{123}-4 \tilde{\tilde{T}}_{127} \\
& -6 \tilde{\tilde{T}}_{158}+\tilde{\tilde{T}}_{234578}-3 \tilde{\tilde{V}}_{43}+2 \tilde{\tilde{V}}_{123}+\tilde{\tilde{V}}_{1345}, \\
& \tilde{\Phi}_{3}^{(\mathcal{P})}=-\tilde{A}_{1}^{M}-3 \tilde{T}_{1}^{M}+\tilde{V}_{1}^{M}+\tilde{\tilde{\tilde{A}}}_{123456}-3 \tilde{\tilde{\tilde{T}}}_{125678}+\tilde{\tilde{\tilde{T}}}_{234578}+\tilde{\tilde{\tilde{V}}}_{123456} ;
\end{aligned}
$$

- the axial-vector interpolating current

$$
\begin{aligned}
\tilde{\omega}_{11}^{(\mathcal{A})} & =2\left[2 m_{c} \tilde{\Phi}_{1}^{(\mathcal{A})}-x m_{N}\left(2 \tilde{\Phi}_{1}^{(\mathcal{A})}+\tilde{\Phi}_{2}^{(\mathcal{A})}\right)+2 m_{N} \tilde{\Phi}_{3}^{(\mathcal{A})}\right] \\
\tilde{\omega}_{12}^{(\mathcal{A})} & =2 m_{N}\left[x^{2} m_{N}^{2} \tilde{\Phi}_{4}^{(\mathcal{A})}+x m_{N} m_{c} \tilde{\Phi}_{5}^{(\mathcal{A})}+2 m_{c}^{2} \tilde{\Phi}_{3}^{(\mathcal{A})}+2 x m_{N}^{2} \tilde{\Phi}_{6}^{(\mathcal{A})}\right] \\
\tilde{\omega}_{13}^{(\mathcal{A})} & =8 m_{N}^{2} m_{c}\left[m_{c}^{2} \tilde{\Phi}_{7}^{(\mathcal{A})}+x m_{N} m_{c} \tilde{\Phi}_{6}^{(\mathcal{A})}+x^{2} m_{N}^{2} \tilde{\Phi}_{8}^{(\mathcal{A})}\right] \\
\tilde{\omega}_{21}^{(\mathcal{A})} & =4 \tilde{\Phi}_{1}^{(\mathcal{A})}, \quad \tilde{\omega}_{23}^{(\mathcal{A})}=8 m_{N}^{2} m_{c}\left[m_{c} \tilde{\Phi}_{7}^{(\mathcal{A})}-x m_{N} \tilde{\Phi}_{8}^{(\mathcal{A})}\right] \\
\tilde{\omega}_{22}^{(\mathcal{A})} & =2 m_{N}\left[2 m_{c} \tilde{\Phi}_{3}^{(\mathcal{A})}-x m_{N} \tilde{\Phi}_{4}^{(\mathcal{A})}+2 m_{N} \tilde{\Phi}_{7}^{(\mathcal{A})}\right]
\end{aligned}
$$




$$
\begin{aligned}
\tilde{\omega}_{31}^{(\mathcal{A})}= & 2 \frac{m_{c}^{2}-q^{2}}{x} \tilde{\Phi}_{1}^{(\mathcal{A})}+m_{N} m_{c} \tilde{\Phi}_{2}^{(\mathcal{A})}+m_{N}^{2} \tilde{\Phi}_{9}^{(\mathcal{A})}-2 x m_{N}^{2} \tilde{\Phi}_{10}^{(\mathcal{A})}-2 \frac{m_{N}^{2}}{x} \tilde{\Phi}_{7}^{(\mathcal{A})}, \\
\tilde{\omega}_{32}^{(\mathcal{A})}= & m_{N}^{2}\left[2\left(q^{2}-x^{2} m_{N}^{2}\right) \tilde{\Phi}_{3}^{(\mathcal{A})}-2 \frac{q^{2}+m_{c}^{2}}{x} \tilde{\Phi}_{7}^{(\mathcal{A})}+x m_{N} m_{c} \tilde{\Phi}_{11}^{(\mathcal{A})}\right. \\
& \left.+m_{c}^{2}\left(\tilde{\Phi}_{5}^{(\mathcal{A})}-2 \tilde{\Phi}_{3}^{(\mathcal{A})}\right)+2 m_{N}\left(m_{c}+x m_{N}\right) \tilde{\Phi}_{8}^{(\mathcal{A})}\right] \\
\tilde{\omega}_{33}^{(\mathcal{A})}= & \frac{4 m_{c}^{2} m_{N}^{2}}{x}\left[\left(m_{c}^{2}-q^{2}\right) \tilde{\Phi}_{7}^{(\mathcal{A})}+x m_{N} m_{c} \tilde{\Phi}_{12}^{(\mathcal{A})}+x^{2} m_{N}^{2} \tilde{\Phi}_{8}^{(\mathcal{A})}\right] \\
\tilde{\omega}_{41}^{(\mathcal{A})}= & 2 m_{N}\left[\left(\tilde{\Phi}_{1}^{(\mathcal{A})}+\tilde{\Phi}_{10}^{(\mathcal{A})}\right)-\frac{\tilde{\Phi}_{3}^{(\mathcal{A})}}{x}\right], \quad \tilde{\omega}_{43}^{(\mathcal{A})}=4 m_{N}^{3} m_{c}^{2} \tilde{\Phi}_{13}^{(\mathcal{A})}, \\
\tilde{\omega}_{42}^{(\mathcal{A})}= & \frac{m_{N}}{x}\left[2\left(m_{c}^{2}+x^{2} m_{N}^{2}-q^{2}\right) \tilde{\Phi}_{3}^{(\mathcal{A})}-x m_{N} m_{c} \tilde{\Phi}_{11}^{(\mathcal{A})}+2 x m_{N}^{2} \tilde{\Phi}_{13}^{(\mathcal{A})}\right] \\
\tilde{\omega}_{51}^{(\mathcal{A})}= & 2 m_{N} \tilde{\Phi}_{2}^{(\mathcal{A})}, \quad \tilde{\omega}_{53}^{(\mathcal{A})}=8 m_{N}^{3} m_{c}\left[m_{c} \tilde{\Phi}_{14}^{(\mathcal{A})}-x m_{N} \tilde{\Phi}_{8}^{(\mathcal{A})}\right] \\
\tilde{\omega}_{52}^{(\mathcal{A})}= & 2 m_{N}^{2}\left[-x m_{N} \tilde{\Phi}_{4}^{(\mathcal{A})}-m_{c}\left(\tilde{\Phi}_{5}^{(\mathcal{A})}+2 \tilde{\Phi}_{3}^{(\mathcal{A})}\right)+2 m_{N} \tilde{\Phi}_{14}^{(\mathcal{A})}\right] \\
& \tilde{\omega}_{61}^{(\mathcal{A})}=0, \quad \tilde{\omega}_{62}^{(\mathcal{A})}=2 m_{N}^{2} \tilde{\Phi}_{4}^{(\mathcal{A})}, \tilde{\omega}_{63}^{(\mathcal{A})}=8 m_{N}^{3} m_{c} \tilde{\Phi}_{8}^{(\mathcal{A})} .
\end{aligned}
$$

The functions $\tilde{\Phi}_{i}^{(\mathcal{A})}$ are given by

$$
\begin{aligned}
& \tilde{\Phi}_{1}^{(\mathcal{A})}=\tilde{A}_{1}+2 \tilde{T}_{1}+\tilde{V}_{1}, \\
& \tilde{\Phi}_{2}^{(\mathcal{A})}=2 \tilde{A}_{3}-2 \tilde{P}_{1}+2 \tilde{S}_{1}-2 \tilde{T}_{1}+\tilde{T}_{123}+\tilde{T}_{127}-2 \tilde{V}_{3}, \\
& \tilde{\Phi}_{3}^{(\mathcal{A})}=\tilde{\tilde{\tilde{A}}}_{123}-\tilde{\tilde{T}}_{123}-\tilde{\tilde{T}}_{127}-\tilde{\tilde{V}}_{123}, \\
& \tilde{\Phi}_{4}^{(\mathcal{A})}=-\tilde{\tilde{A}}_{34}+\tilde{\tilde{A}}_{1345}-2 \tilde{\tilde{P}}_{21}-2 \tilde{\tilde{S}}_{12}-2 \tilde{\tilde{T}}_{127}+2 \tilde{\tilde{T}}_{158}-\tilde{\tilde{T}}_{234578}-\tilde{\tilde{V}}_{43}+\tilde{\tilde{V}}_{1345}, \\
& \tilde{\Phi}_{5}^{(\mathcal{A})}=-\tilde{\tilde{A}}_{34}-2 \tilde{\tilde{A}}_{123}-\tilde{\tilde{A}}_{1345}+4 \tilde{\tilde{T}}_{127}-4 \tilde{\tilde{T}}_{158}+2 \tilde{\tilde{T}}_{234578}-\tilde{\tilde{V}}_{43}+2 \tilde{\tilde{V}}_{123}-\tilde{\tilde{V}}_{1345}, \\
& \tilde{\Phi}_{6}^{(\mathcal{A})}=\tilde{\tilde{A}}_{1}^{M}+\tilde{T}_{1}^{M}+\tilde{\tilde{V}}_{1}^{M}-\tilde{\tilde{\tilde{A}}}_{123456}+\tilde{\tilde{\tilde{T}}}_{125678}-\tilde{\tilde{T}}_{234578}+\tilde{\tilde{V}}_{123456}, \\
& \tilde{\Phi}_{7}^{(\mathcal{A})}=-\tilde{\tilde{A}}_{1}^{M}-2 \tilde{T}_{1}^{M}-\tilde{V}_{1}^{M}+\tilde{\tilde{T}}_{234578}, \\
& \tilde{\Phi}_{8}^{(\mathcal{A})}=\tilde{\tilde{\tilde{A}}}_{123456}-2 \tilde{\tilde{\tilde{T}}}_{125678}+\tilde{\tilde{T}}_{234578}-\tilde{\tilde{\tilde{V}}}_{123456}, \\
& \tilde{\Phi}_{9}^{(\mathcal{A})}=-2 \tilde{\tilde{A}}_{34}-2 \tilde{\tilde{A}}_{123}-2 \tilde{\tilde{P}}_{21}-2 \tilde{\tilde{S}}_{12}+2 \tilde{\tilde{T}}_{127}-2 \tilde{\tilde{T}}_{158}+\tilde{\tilde{T}}_{234578}-2 \tilde{\tilde{V}}_{43}+2 \tilde{\tilde{V}}_{123}, \\
& \tilde{\Phi}_{10}^{(\mathcal{A})}=\tilde{A}_{123}-\tilde{T}_{123}-\tilde{T}_{127}-\tilde{V}_{123},
\end{aligned}
$$




$$
\begin{aligned}
& \tilde{\Phi}_{11}^{(\mathcal{A})}=2 \tilde{\tilde{A}}_{34}+2 \tilde{\tilde{\tilde{P}}}_{21}+2 \tilde{\tilde{\tilde{S}}}_{12}+2 \tilde{\tilde{T}}_{123}+2 \tilde{\tilde{T}}_{158}-\tilde{\tilde{T}}_{234578}+2 \tilde{\tilde{V}}_{43}, \\
& \tilde{\Phi}_{12}^{(\mathcal{A})}=\tilde{\tilde{T}}_{1}^{M}+\tilde{\tilde{T}}_{125678}-\tilde{\tilde{T}}_{234578}, \\
& \tilde{\Phi}_{13}^{(\mathcal{A})}=-\tilde{A}_{1}^{M}-2 \tilde{T}_{1}^{M}-\tilde{\tilde{V}}_{1}^{M}-\tilde{\tilde{\tilde{A}}}_{123456}+2 \tilde{\tilde{\tilde{T}}}_{125678}+\tilde{\tilde{V}}_{123456}, \\
& \tilde{\Phi}_{14}^{(\mathcal{A})}=\tilde{T}_{1}^{M}+\tilde{\tilde{\tilde{A}}}_{123456}-\tilde{\tilde{T}}_{125678}-\tilde{\tilde{V}}_{123456} .
\end{aligned}
$$

\section{B.3. axial-vector transition current}

The invariant amplitudes $\bar{\Pi}_{j}^{(i)}\left((P-q)^{2}, q^{2}\right)$ for the correlation function with the axialvector transition current $j_{\mu 5}$ are given by (26) where $m_{c} / 4 \rightarrow 1 / 4$, with $i=\mathcal{P}, \mathcal{A}$ and $j=1,2, \ldots 6$. The coefficient functions $\bar{\omega}_{j n}^{(i)}$ can be obtained from $\tilde{\omega}_{j n}^{(i)}$ in the above subsection by changing the sign at $m_{c}$ and at $\tilde{\omega}_{2 n}^{(\mathcal{P})}, \tilde{\omega}_{3 n}^{(\mathcal{P})}, \tilde{\omega}_{6 n}^{(\mathcal{P})}, \tilde{\omega}_{1 n}^{(\mathcal{A})}, \tilde{\omega}_{4 n}^{(\mathcal{A})}$ and $\tilde{\omega}_{5 n}^{(\mathcal{A})}$.

\section{Correlation function in the LCSR for $\Sigma_{c} \rightarrow N$ form factors}

\section{C.1. pseudoscalar transition current}

The invariant amplitudes $\Pi_{j}^{(i)}\left((P-q)^{2}, q^{2}\right)$ for the correlation function with the pseudoscalar transition current $j_{5}$ are given by Eq. (26) with $i=\mathcal{I}, \mathcal{T}$ and $j=1,2$. Here we specify the corresponding coefficient funcitons for:

- Ioffe current:

The functions $\omega_{j n}^{(\mathcal{I})}$ can be obtained from $\omega_{j n}^{(\mathcal{A})}$ given in previous App.B by changing the sign of the terms involving scalar $S_{1,2}\left(x_{i}\right)$, pseudoscalar $P_{1,2}\left(x_{i}\right)$ and tensor $T_{1, \ldots, 8}\left(x_{i}\right)$ DA's and also $m_{c} \rightarrow-m_{c}$;

- tensor interpolating current:

$$
\begin{aligned}
\omega_{11}^{(\mathcal{T})}= & 2\left[4 \frac{m_{c}^{2}-q^{2}}{x} \Phi_{1}^{(\mathcal{T})}+m_{N} m_{c} \Phi_{2}^{(\mathcal{T})}+2 x m_{N}^{2}\left(\Phi_{1}^{(\mathcal{T})}+\Phi_{3}^{(\mathcal{T})}\right)\right. \\
& \left.+m_{N}^{2} \Phi_{4}^{(\mathcal{T})}+\frac{4 m_{N}^{2}}{x} \Phi_{5}^{(\mathcal{T})}\right], \\
\omega_{12}^{(\mathcal{T})}= & 2 m_{N}^{2}\left[4\left(x^{2} m_{N}^{2}-q^{2}\right) \Phi_{6}^{(\mathcal{T})}+x m_{N} m_{c} \Phi_{7}^{(\mathcal{T})}+m_{c}^{2} \Phi_{4}^{(\mathcal{T})}-\frac{2}{x}\left(2\left(q^{2}+m_{c}^{2}\right)\right.\right. \\
& \left.\left.+x^{2} m_{N}^{2}\right) \Phi_{5}^{(\mathcal{T})}-6 x m_{N}^{2} \Phi_{8}^{(\mathcal{T})}\right], \\
\omega_{13}^{(\mathcal{T})}= & \frac{8 m_{N}^{2} m_{c}^{2}}{x}\left[2\left(q^{2}-m_{c}^{2}\right) \Phi_{5}^{(\mathcal{T})}+x m_{N} m_{c} \Phi_{9}^{(\mathcal{T})}-x^{2} m_{N}^{2}\left(3 \Phi_{8}^{(\mathcal{T})}+\Phi_{5}^{(\mathcal{T})}\right)\right],
\end{aligned}
$$




$$
\begin{aligned}
\omega_{21}^{(\mathcal{T})}= & 4 m_{N}\left[-\Phi_{1}^{(\mathcal{T})}+\Phi_{3}^{(\mathcal{T})}-\frac{2}{x} \Phi_{6}^{(\mathcal{T})}\right], \quad \omega_{23}^{(\mathcal{T})}=8 m_{N}^{3} m_{c}^{2}\left[\Phi_{5}^{(\mathcal{T})}-3 \Phi_{8}^{(\mathcal{T})}\right] \\
\omega_{22}^{(\mathcal{T})}=\frac{2 m_{N}}{x}[ & 4\left(m_{c}^{2}+x^{2} m_{N}^{2}-q^{2}\right) \Phi_{6}^{(\mathcal{T})}+x m_{N} m_{c} \Phi_{7}^{(\mathcal{T})} \\
& \left.\quad-2 x m_{N}^{2}\left(3 \Phi_{8}^{(\mathcal{T})}+\Phi_{5}^{(\mathcal{T})}\right)\right]
\end{aligned}
$$

where the functions $\Phi_{i}^{(\mathcal{T})}$ are

$$
\begin{aligned}
& \Phi_{1}^{(\mathcal{T})}=\tilde{V}_{1}-\tilde{A}_{1}, \quad \Phi_{3}^{(\mathcal{T})}=\tilde{V}_{123}+\tilde{A}_{123}, \\
& \Phi_{2}^{(\mathcal{T})}=6 \tilde{P}_{1}+6 \tilde{S}_{1}-6 \tilde{T}_{1}+12 \tilde{T}_{7}+\tilde{T}_{123}+5 \tilde{T}_{127}, \\
& \Phi_{4}^{(\mathcal{T})}=3 \tilde{\tilde{A}}_{34}+2 \tilde{\tilde{A}}_{123}+3 \tilde{\tilde{A}}_{1345}-3 \tilde{\tilde{V}}_{43}+2 \tilde{\tilde{V}}_{123}-3 \tilde{\tilde{V}}_{1345}, \\
& \Phi_{5}^{(\mathcal{T})}=\tilde{V}_{1}^{M}-\tilde{A}_{1}^{M}, \quad \Phi_{6}^{(\mathcal{T})}=\tilde{\tilde{V}}_{123}+\tilde{\tilde{A}}_{123}, \\
& \Phi_{7}^{(\mathcal{T})}=-6 \tilde{\tilde{P}}_{21}+6 \tilde{\tilde{S}}_{12}+12 \tilde{\tilde{T}}_{78}+2 \tilde{\tilde{T}}_{123}+4 \tilde{\tilde{T}}_{127}+6 \tilde{\tilde{T}}_{158}-\tilde{\tilde{T}}_{234578}, \\
& \Phi_{8}^{(\mathcal{T})}=\tilde{\tilde{\tilde{V}}}_{123456}+\tilde{\tilde{\tilde{A}}}_{123456}, \quad \Phi_{9}^{(\mathcal{T})}=3 \tilde{\tilde{T}}_{1}^{M}+3 \tilde{\tilde{\tilde{T}}}_{125678}-\tilde{\tilde{T}}_{234578} .
\end{aligned}
$$

\section{C.2. vector transition current}

The invariant amplitudes $\tilde{\Pi}_{j}^{(i)}\left((P-q)^{2}, q^{2}\right)$, with $i=\mathcal{I}, \mathcal{T}$ and $j=1,2, \ldots 6$, for the correlation function with the vector transition current $j_{\mu}$ are given by Eq. (26) with the replacement $m_{c} / 4 \rightarrow 1 / 4$, where the coefficient $\tilde{\omega}_{j n}^{(i)}$ functions are given below for:

- Ioffe current:

The functions $\tilde{\omega}_{j n}^{(\mathcal{I})}$ can be obtained from $\tilde{\omega}_{j n}^{(\mathcal{A})}$ presented in App. B by changing the sign of the terms involving vector $V_{1, \ldots, 6}\left(x_{i}\right)$ and axial-vector $A_{1, \ldots, 6}\left(x_{i}\right)$ DA's as well as $m_{c} \rightarrow-m_{c}$;

- tensor interpolating current

$$
\begin{aligned}
\tilde{\omega}_{11}^{(\mathcal{T})}= & 4\left[-4 m_{c} \tilde{\Phi}_{1}^{(\mathcal{T})}+x m_{N} \tilde{\Phi}_{2}^{(\mathcal{T})}+4 m_{N} \tilde{\Phi}_{3}^{(\mathcal{T})}\right], \\
\tilde{\omega}_{12}^{(\mathcal{T})}= & 4 m_{N}\left[4 q^{2} \tilde{\Phi}_{3}^{(\mathcal{T})}-x^{2} m_{N}^{2}\left(4 \tilde{\Phi}_{3}^{(\mathcal{T})}+2 \tilde{\Phi}_{4}^{(\mathcal{T})}+3 \tilde{\Phi}_{5}^{(\mathcal{T})}\right)+2 x m_{N} m_{c}\left(\tilde{\Phi}_{6}^{(\mathcal{T})}-2 \tilde{\Phi}_{7}^{(\mathcal{T})}\right)\right. \\
& \left.+2 x m_{N}^{2}\left(\tilde{\Phi}_{8}^{(\mathcal{T})}+\tilde{\Phi}_{9}^{(\mathcal{T})}\right)\right], \\
\tilde{\omega}_{13}^{(\mathcal{T})}= & 16 m_{N}^{2}\left[x m_{N} m_{c}^{2} \tilde{\Phi}_{8}^{(\mathcal{T})}+2 x^{2} m_{N}^{2} m_{c} \tilde{\Phi}_{10}^{(\mathcal{T})}+x m_{N}\left(2\left(q^{2}-x^{2} m_{N}^{2}\right)-m_{c}^{2}\right) \tilde{\Phi}_{9}^{(\mathcal{T})}\right. \\
& \left.+2 m_{c}^{3} \Phi_{11}^{(\mathcal{T})}\right], \\
& \\
\tilde{\omega}_{21}^{(\mathcal{T})}= & 0, \quad \tilde{\omega}_{23}^{(\mathcal{T})}=32 m_{N}^{2}\left[\left(x^{2} m_{N}^{2}-q^{2}\right) \tilde{\Phi}_{9}^{(\mathcal{T})}-x m_{N} m_{c} \tilde{\Phi}_{10}^{(\mathcal{T})}\right], \\
\tilde{\omega}_{22}^{(\mathcal{T})}= & 4 m_{N}\left[4 m_{c} \tilde{\Phi}_{12}^{(\mathcal{T})}+x m_{N}\left(2 \tilde{\Phi}_{4}^{(\mathcal{T})}+3 \tilde{\Phi}_{5}^{(\mathcal{T})}\right)-8 m_{N} \tilde{\Phi}_{9}^{(\mathcal{T})}\right],
\end{aligned}
$$




$$
\begin{aligned}
& \tilde{\omega}_{31}^{(\mathcal{T})}=2 m_{N}\left[2 m_{c}\left(\tilde{\Phi}_{1}^{(\mathcal{T})}-\tilde{\Phi}_{13}^{(\mathcal{T})}\right)+x m_{N}\left(\tilde{\Phi}_{2}^{(\mathcal{T})}+4 \tilde{\Phi}_{14}^{(\mathcal{T})}\right)\right. \\
& \left.+2 m_{N}\left(-2\left(\tilde{\Phi}_{3}^{(\mathcal{T})}+\tilde{\Phi}_{4}^{(\mathcal{T})}\right)+\tilde{\Phi}_{5}^{(\mathcal{T})}\right)\right] \\
& \tilde{\omega}_{32}^{(\mathcal{T})}=2 m_{N}^{2}\left[-x m_{N} m_{c}\left(\tilde{\Phi}_{6}^{(\mathcal{T})}+2 \tilde{\Phi}_{12}^{(\mathcal{T})}\right)+4 m_{N} m_{c} \tilde{\Phi}_{10}^{(\mathcal{T})}+2 x m_{N}^{2}\left(\tilde{\Phi}_{8}^{(\mathcal{T})}+\tilde{\Phi}_{9}^{(\mathcal{T})}\right)\right. \\
& \left.+m_{c}^{2}\left(-2 \tilde{\Phi}_{4}^{(\mathcal{T})}+5 \tilde{\Phi}_{5}^{(\mathcal{T})}-\frac{8}{x} \tilde{\Phi}_{9}^{(\mathcal{T})}\right)+4\left(x^{2} m_{N}^{2}-q^{2}\right) \tilde{\Phi}_{3}^{(\mathcal{T})}\right] \\
& \tilde{\omega}_{33}^{(\mathcal{T})}=\frac{8 m_{N}^{2} m_{c}^{2}}{x}\left[2\left(x^{2} m_{N}^{2}-q^{2}+m_{c}^{2}\right) \tilde{\Phi}_{9}^{(\mathcal{T})}+x^{2} m_{N}^{2}\left(\tilde{\Phi}_{8}^{(\mathcal{T})}+\tilde{\Phi}_{9}^{(\mathcal{T})}\right)\right. \\
& \left.-x m_{N} m_{c}\left(\tilde{\Phi}_{10}^{(\mathcal{T})}+\tilde{\Phi}_{11}^{(\mathcal{T})}\right)\right] \text {, } \\
& \tilde{\omega}_{41}^{(\mathcal{T})}=\frac{2 m_{N}}{x}\left[4 \tilde{\Phi}_{3}^{(\mathcal{T})}-x\left(\tilde{\Phi}_{2}^{(\mathcal{T})}+4 \tilde{\Phi}_{14}^{(\mathcal{T})}\right)\right], \\
& \tilde{\omega}_{42}^{(\mathcal{T})}=\frac{2 m_{N}}{x}\left[4\left(q^{2}-x^{2} m_{N}^{2}-m_{c}^{2}\right) \tilde{\Phi}_{3}^{(\mathcal{T})}+x m_{N} m_{c}\left(\tilde{\Phi}_{6}^{(\mathcal{T})}+2 \tilde{\Phi}_{7}^{(\mathcal{T})}\right)\right. \\
& \left.-2 x m_{N}^{2}\left(\tilde{\Phi}_{8}^{(\mathcal{T})}+\tilde{\Phi}_{9}^{(\mathcal{T})}\right)\right], \\
& \tilde{\omega}_{43}^{(\mathcal{T})}=-8 m_{N}^{3} m_{c}^{2}\left(\tilde{\Phi}_{8}^{(\mathcal{T})}+\tilde{\Phi}_{9}^{(\mathcal{T})}\right) \\
& \tilde{\omega}_{51}^{(\mathcal{T})}=-4 m_{N}\left[\tilde{\Phi}_{2}^{(\mathcal{T})}+\frac{4 \tilde{\Phi}_{3}^{(\mathcal{T})}}{x}\right] \\
& \tilde{\omega}_{52}^{(\mathcal{T})}=\frac{4 m_{N}}{x}\left[4\left(m_{c}^{2}-q^{2}\right) \tilde{\Phi}_{3}^{(\mathcal{T})}+x^{2} m_{N}^{2}\left(4 \tilde{\Phi}_{3}^{(\mathcal{T})}+2 \tilde{\Phi}_{4}^{(\mathcal{T})}+3 \tilde{\Phi}_{5}^{(\mathcal{T})}\right)\right. \\
& \left.-2 x m_{N} m_{c} \tilde{\Phi}_{6}^{(\mathcal{T})}-2 x m_{N}^{2}\left(\tilde{\Phi}_{8}^{(\mathcal{T})}+\tilde{\Phi}_{9}^{(\mathcal{T})}\right)\right], \\
& \tilde{\omega}_{53}^{(\mathcal{T})}=16 m_{N}^{3}\left[2\left(x^{2} m_{N}^{2}-q^{2}\right) \tilde{\Phi}_{9}^{(\mathcal{T})}+m_{c}^{2}\left(3 \tilde{\Phi}_{9}^{(\mathcal{T})}-\tilde{\Phi}_{8}^{(\mathcal{T})}\right)-2 x m_{N} m_{c} \tilde{\Phi}_{10}^{(\mathcal{T})}\right], \\
& \tilde{\omega}_{61}^{(\mathcal{T})}=0, \quad \tilde{\omega}_{62}^{(\mathcal{T})}=4 m_{N}^{2}\left[-2 \tilde{\Phi}_{4}^{(\mathcal{T})}-3 \tilde{\Phi}_{5}^{(\mathcal{T})}+\frac{8}{x} \tilde{\Phi}_{9}^{(\mathcal{T})}\right] \\
& \tilde{\omega}_{63}^{(\mathcal{T})}=\frac{32 m_{N}^{2}}{x}\left[\left(q^{2}-x^{2} m_{N}^{2}-m_{c}^{2}\right) \tilde{\Phi}_{9}^{(\mathcal{T})}+x m_{N} m_{c} \tilde{\Phi}_{10}^{(\mathcal{T})}\right],
\end{aligned}
$$

where the functions $\tilde{\Phi}_{i}^{(\mathcal{T})}$ are

$$
\begin{aligned}
& \tilde{\Phi}_{1}^{(\mathcal{T})}=\tilde{V}_{1}-\tilde{A}_{1}, \quad \tilde{\Phi}_{2}^{(\mathcal{T})}=2 \tilde{P}_{1}+2 \tilde{S}_{1}-2 \tilde{T}_{1}+4 \tilde{T}_{7}-\tilde{T}_{123}+3 \tilde{T}_{127}, \\
& \tilde{\Phi}_{3}^{(\mathcal{T})}=\tilde{\tilde{T}}_{123}-\tilde{\tilde{T}}_{127}, \quad \tilde{\Phi}_{4}^{(\mathcal{T})}=\tilde{\tilde{P}}_{21}-\tilde{\tilde{S}}_{12}-2 \tilde{\tilde{T}}_{78}-\tilde{\tilde{T}}_{123}-\tilde{\tilde{T}}_{158},
\end{aligned}
$$




$$
\begin{aligned}
& \tilde{\Phi}_{5}^{(\mathcal{T})}=\tilde{\tilde{T}}_{234578}, \quad \tilde{\Phi}_{6}^{(\mathcal{T})}=\tilde{\tilde{V}}_{43}+\tilde{\tilde{V}}_{1345}-\tilde{\tilde{A}}_{34}-\tilde{\tilde{A}}_{1345}, \\
& \tilde{\Phi}_{7}^{(\mathcal{T})}=\tilde{\tilde{V}}_{123}+\tilde{\tilde{A}}_{123}, \quad \tilde{\Phi}_{8}^{(\mathcal{T})}=\tilde{T}_{1}^{M}+\tilde{\tilde{\tilde{T}}}_{125678}, \\
& \tilde{\Phi}_{9}^{(\mathcal{T})}=\tilde{\tilde{\tilde{T}}}_{234578}, \quad \tilde{\Phi}_{10}^{(\mathcal{T})}=\tilde{\tilde{\tilde{V}}}_{123456}+\tilde{\tilde{\tilde{A}}}_{123456}, \\
& \tilde{\Phi}_{11}^{(\mathcal{T})}=\tilde{V}_{1}^{M}-\tilde{A}_{1}^{M}, \quad \tilde{\Phi}_{12}^{(\mathcal{T})}=\tilde{\tilde{V}}_{123}+\tilde{\tilde{A}}_{123}, \\
& \tilde{\Phi}_{13}^{(\mathcal{T})}=\tilde{V}_{123}+\tilde{A}_{123}, \quad \tilde{\Phi}_{14}^{(\mathcal{T})}=\tilde{T}_{123}-\tilde{T}_{127} .
\end{aligned}
$$

\section{C.3. axial-vector transition current}

The invariant amplitudes $\bar{\Pi}_{j}^{(i)}\left((P-q)^{2}, q^{2}\right)$, with $i=\mathcal{I}, \mathcal{T}$ and $j=1,2, \ldots 6$, for the correlation function with the axial-vector transition current $j_{\mu 5}$ are given by Eq. (26) with the replacement $m_{c} / 4 \rightarrow 1 / 4$. The coefficient functions $\bar{\omega}_{j n}^{(i)}$ can be obtained from $\tilde{\omega}_{j n}^{(i)}$ in the above subsection by changing the sign for $\tilde{\omega}_{2 n}^{(\mathcal{T})}, \tilde{\omega}_{3 n}^{(\mathcal{T})}, \tilde{\omega}_{6 n}^{(\mathcal{T})}, \tilde{\omega}_{1 n}^{(\mathcal{I})}, \tilde{\omega}_{4 n}^{(\mathcal{I})}$ and $\tilde{\omega}_{5 n}^{(\mathcal{I})}$ together with $m_{c} \rightarrow-m_{c}$.

\section{Two-point sum rules}

Here we present the expressions for the spectral densities in the two-point sum rules for the decay constants of charmed baryons:

\section{D.1. $\Lambda_{c}$ baryon}

$$
\begin{aligned}
\operatorname{Im} F_{1}^{(\mathcal{P})}(s)= & \frac{m_{c}^{4}}{512 \pi^{3}}\left[\left(1-\tau^{2}\right)\left(1-\frac{8}{\tau}+\frac{1}{\tau^{2}}\right)-12 \ln \tau\right] \\
& +\frac{1}{768 \pi^{2}}\left\langle\alpha_{s} G^{2}\right\rangle(1-\tau)(1+5 \tau)+\pi \frac{\langle\bar{q} q\rangle^{2}}{6} \delta\left(s-m_{c}^{2}\right), \\
\operatorname{Im} F_{2}^{(\mathcal{P})}(s)= & \frac{m_{c}^{5}}{128 \pi^{3}}\left[(1-\tau)\left(1+\frac{10}{\tau}+\frac{1}{\tau^{2}}\right)+6\left(1+\frac{1}{\tau}\right) \ln \tau\right] \\
& +\frac{m_{c}}{384 \pi^{2}}\left\langle\alpha_{s} G^{2}\right\rangle\left[(1-\tau)\left(7+\frac{2}{\tau}\right)+6 \ln \tau\right] \\
& +\pi m_{c} \frac{\langle\bar{q} q\rangle^{2}}{6} \delta\left(s-m_{c}^{2}\right), \\
& \frac{\left(5+2 b+5 b^{2}\right) m_{c}^{4}}{2048 \pi^{3}}\left[\left(1-\tau^{2}\right)\left(1-\frac{8}{\tau}+\frac{1}{\tau^{2}}\right)-12 \ln \tau\right] \\
\operatorname{Im} \tilde{F}_{1}(s)= & \frac{\left(5-4 b-b^{2}\right) m_{c}}{96 \pi}\langle\bar{q} q\rangle(1-\tau)^{2} \\
+ & \frac{\left\langle\alpha_{s} G^{2}\right\rangle}{3072 \pi^{2}}(1-\tau)\left[\left(5+2 b+5 b^{2}\right)+3\left(7+6 b+7 b^{2}\right) \tau\right]
\end{aligned}
$$




$$
\begin{aligned}
+ & \frac{m_{0}^{2}\langle\bar{q} q\rangle(1-b)}{384 \pi} \frac{\tau}{m_{c}}(11 \tau-6+b(7 \tau-6)) \\
+ & \pi \frac{\langle\bar{q} q\rangle^{2}}{72} \delta\left(s-m_{c}^{2}\right)\left(11+2 b+3 b^{2}\right), \\
\operatorname{Im} \tilde{F}_{2}(s)= & \frac{\left(11+2 b-13 b^{2}\right) m_{c}^{5}}{1536 \pi^{3}}\left[(1-\tau)\left(1+\frac{10}{\tau}+\frac{1}{\tau^{2}}\right)+6\left(1+\frac{1}{\tau}\right) \ln \tau\right] \\
& -\frac{5-4 b-b^{2}}{96 \pi} s\langle\bar{q} q\rangle(1-\tau)^{2}-\frac{m_{0}^{2}\langle\bar{q} q\rangle(1-b)}{384 \pi}[\tau-6+b(5 \tau-6)] \\
+ & \frac{(1-b) m_{c}\left\langle\alpha_{s} G^{2}\right\rangle}{4608 \pi^{2}}\left[(1-\tau)\left(2(11+13 b) \frac{1}{\tau}+(89+79 b)\right)+72(1+b) \ln \tau\right] \\
+ & \pi \frac{\langle\bar{q} q\rangle^{2}}{24} \delta\left(s-m_{c}^{2}\right)\left(5+2 b+5 b^{2}\right) m_{c},
\end{aligned}
$$

where for brevity we denote $m_{c}^{2} / s=\tau$ and use the standard notations for the vacuum condensate densities. The above relations for $\operatorname{Im} \tilde{F}_{1,2}(s)$ are used at $b=-1 / 5$. Hereafter the integration convention $\int_{m_{c}^{2}}^{\infty} d s \delta\left(s-m_{c}^{2}\right)=1$ is implied.

\section{D.2. $\Sigma_{c}$ baryon}

$$
\begin{aligned}
\operatorname{Im} \bar{F}_{1}(s)= & \frac{\left(5+2 b+5 b^{2}\right) m_{c}^{4}}{512 \pi^{3}}\left[-\frac{\tau^{2}}{4}+2 \tau-\frac{2}{\tau}+\frac{1}{4 \tau^{2}}-3 \ln \tau\right] \\
& -\frac{3 m_{c}}{32 \pi}\langle\bar{q} q\rangle\left(1-b^{2}\right)(1-\tau)^{2}+\pi \frac{\langle\bar{q} q\rangle^{2}}{24} \delta\left(s-m_{c}^{2}\right)(1-b)^{2} \\
& -\frac{\left\langle\alpha_{s} G^{2}\right\rangle}{3072 \pi^{2}} \tau(1-\tau)\left[\left(1+b^{2}\right)\left(11-\frac{5}{\tau}\right)+2 b\left(7-\frac{1}{\tau}\right)\right] \\
& +\frac{m_{0}^{2}\langle\bar{q} q\rangle\left(1-b^{2}\right)}{128 \pi} \frac{1}{m_{c}} 13 \tau^{2}\left(1-\frac{6}{13 \tau}\right), \\
\operatorname{Im} \bar{F}_{2}(s)= & \frac{(1-b)^{2} m_{c}^{5}}{512 \pi^{3} \tau^{2}}\left[(1-\tau)\left(1+10 \tau+\tau^{2}\right)+6 \tau(1+\tau) \ln \tau\right] \\
& -\frac{3 m_{c}^{2}}{32 \pi}\langle\bar{q} q\rangle\left(1-b^{2}\right) \tau\left(1-\frac{1}{\tau}\right)^{2}+\frac{m_{0}^{2}\langle\bar{q} q\rangle\left(1-b^{2}\right)}{128 \pi}(6+\tau) \\
& -\frac{(1-b)^{2} m_{c}\left\langle\alpha_{s} G^{2}\right\rangle}{1536 \pi^{2}}(1-\tau)\left(5-\frac{2}{\tau}\right) \\
& +\pi \frac{\langle\bar{q} q\rangle^{2}}{24} \delta\left(s-m_{c}^{2}\right)\left(5+2 b+5 b^{2}\right) m_{c},
\end{aligned}
$$

used for $b= \pm 1$.

\section{E. Double spectral representations}

Here we collect the double dispersion relations for the master integrals with the powers $n=2,3$ in denominators. 


$$
\begin{aligned}
& \int_{0}^{1} d x \frac{x^{k}}{D^{2}}=-\frac{1}{\pi} \int_{m_{c}^{2}}^{\infty} \frac{d s}{s-(P-q)^{2}} \int_{t_{1}(s)}^{t_{2}(s)} \frac{d s^{\prime}}{s^{\prime}-q^{2}}\left\{\sum_{j=2}^{k}(-1)^{k+1+j / 2} \frac{1+(-1)^{j}}{2}\right. \\
& \times \frac{j-1}{\left(2 m_{N}^{2}\right)^{k-1}} C_{k}^{j}\left[\bar{s}\left(s^{\prime}\right)\right]^{k-j}\left[\kappa\left(s^{\prime}, t_{1}, t_{2}\right)\right]^{\frac{j-3}{2}} \theta(k-2) \\
& +\frac{(-1)^{k}}{\left(2 m_{N}^{2}\right)^{k-1}} \frac{\left[\bar{s}\left(s^{\prime}\right)\right]^{k}}{\left[\kappa\left(s^{\prime}, t_{1}, t_{2}\right)\right]^{3 / 2}} \\
& -\frac{(-1)^{k}}{\left(2 m_{N}^{2}\right)^{k-1}}\left[\left(\frac{\left[\bar{s}\left(t_{1}\right)\right]^{k}}{t_{2}-t_{1}} \delta\left(s^{\prime}-t_{1}\right) X_{1}\left(t_{1}, t_{2}\right)\right)-\left(t_{1} \leftrightarrow t_{2}\right)\right] \\
& \left.-\pi \delta\left(m_{c}^{2}-s\right) \delta\left(m_{c}^{2}+m_{N}^{2}-s^{\prime}\right)\right\}+\ldots, \\
& \int_{0}^{1} d x \frac{x^{k}}{D^{3}}=\frac{1}{2 \pi} \int_{m_{c}^{2}}^{\infty} \frac{d s}{s-(P-q)^{2}} \int_{t_{1}(s)}^{t_{2}(s)} \frac{d s^{\prime}}{s^{\prime}-q^{2}}\left\{\sum_{j=4}^{k}(-1)^{k+j / 2} \frac{1+(-1)^{j}}{2} C_{k}^{j}\right. \\
& \cdot \frac{(j-1)(j-3)}{\left(2 m_{N}^{2}\right)^{k-2}}\left[\bar{s}\left(s^{\prime}\right)\right]^{k-j}\left[\kappa\left(s^{\prime}, t_{1}, t_{2}\right)\right]^{\frac{j-5}{2}} \theta(k-4) \\
& +\frac{(-1)^{k} C_{k}^{2}}{\left(2 m_{N}^{2}\right)^{k-2}} \theta(k-2)\left[\frac{\left[\bar{s}\left(s^{\prime}\right)\right]^{k-2}}{\left[\kappa\left(s^{\prime}, t_{1}, t_{2}\right)\right]^{3 / 2}}\right. \\
& \left.-\left(\frac{\left[\bar{s}\left(t_{1}\right)\right]^{k-2}}{t_{2}-t_{1}} \delta\left(s^{\prime}-t_{1}\right) X_{1}\left(t_{1}, t_{2}\right)\right)+\left(t_{1} \leftrightarrow t_{2}\right)\right] \\
& +3 \frac{(-1)^{k}}{\left(2 m_{N}^{2}\right)^{k-2}}\left\{\frac{\left[\bar{s}\left(s^{\prime}\right)\right]^{k}}{\left[\kappa\left(s^{\prime}, t_{1}, t_{2}\right)\right]^{5 / 2}}\right. \\
& -\left(\frac{\left[\bar{s}\left(t_{1}\right)\right]^{k}}{\left(t_{2}-t_{1}\right)^{3}} \delta\left(s^{\prime}-t_{1}\right) X_{2}\left(t_{1}, t_{2}\right)\right)+\left(t_{1} \leftrightarrow t_{2}\right) \\
& \left.+\left[\frac{\left[\bar{s}\left(t_{1}\right)\right]^{k}}{\left(t_{2}-t_{1}\right)^{2}} \xi\left(s, s^{\prime}, t_{1}\right) X_{1}\left(t_{1}, t_{2}\right)\right]-\left[t_{1} \leftrightarrow t_{2}\right]\right\} \\
& +\frac{\pi}{2} \frac{(-1)^{k}}{\left(2 m_{N}^{2}\right)^{k}}\left(-2 \delta^{\prime}\left(m_{c}^{2}-s\right) \delta\left(m_{c}^{2}+m_{N}^{2}-s^{\prime}\right)\left(-2 m_{N}^{2}\right)^{k}\right. \\
& +k(k-3) \delta\left(m_{c}^{2}-s\right) \delta\left(m_{c}^{2}+m_{N}^{2}-s^{\prime}\right)\left(-2 m_{N}^{2}\right)^{k-1} \theta(k-1) \\
& \left.\left.+\delta\left(m_{c}^{2}-s\right) \delta^{(2)}\left(\bar{s}\left(s^{\prime}\right)+2 m_{N}^{2}\right)\left[\bar{s}\left(s^{\prime}\right)+2 m_{N}^{2}\right]^{k}\left[\bar{s}\left(s^{\prime}\right)+4 m_{N}^{2}\right]\right)\right\} \\
& +\ldots \text {. }
\end{aligned}
$$

In the above, the ellipses denote the terms that vanish after double Borel transformation and are therefore inessential; $t_{1,2}$ are the functions of $s$ defined in (53), $\theta(k-a)=1(0)$ at $k \geq a(k<a), \bar{s}(y)=s-y-m_{N}^{2}$ and $\kappa(a, b, c)=(a-b)(c-a)$. The auxiliary functions 
entering the above expressions are defined as

$$
\begin{aligned}
X_{1}(a, b) & =\int_{a}^{b} \frac{d \sigma}{[\kappa(\sigma, a, b)]^{3 / 2}}(b-\sigma), \\
X_{2}(a, b) & =\int_{a}^{b} \frac{d \sigma}{[\kappa(\sigma, a, b)]^{5 / 2}}(b-\sigma)^{2}(2 \sigma-3 a+b), \\
\xi\left(s, s^{\prime}, a\right) & =\delta^{\prime}\left(s^{\prime}-a\right)+\frac{k \theta(k-1)}{\bar{s}(a)} \delta\left(s^{\prime}-a\right) .
\end{aligned}
$$

\section{References}

[1] T. Aaltonen et al. [CDF Collaboration], Phys. Rev. D79 (2009) 032001; arXiv:1107.3753 [hep-ex]; B. Adeva et al. [The LHCb Collaboration], arXiv:0912.4179 [hep-ex]; G. Graziani et al. [on behalf of the LHCb Collaboration], arXiv:1107.2328 [hep-ex].

[2] U. Wiedner, Prog. Part. Nucl. Phys. 66 (2011) 477.

[3] I. I. Balitsky, V. M. Braun and A. V. Kolesnichenko, Nucl. Phys. B 312 (1989) 509; V. M. Braun and I. E. Filyanov, Z. Phys. C 44 (1989) 157; V. L. Chernyak and I. R. Zhitnitsky, Nucl. Phys. B 345 (1990) 137.

[4] V. M. Belyaev, A. Khodjamirian and R. Rückl, Z. Phys. C60 (1993) 349; A. Khodjamirian, R. Rückl, S. Weinzierl and O. I. Yakovlev, Phys. Lett. B 410 (1997) 275; E. Bagan, P. Ball and V. M. Braun, Phys. Lett. B 417 (1998) 154; P. Ball, JHEP 9809 (1998) 005.

[5] V. Braun, R. J. Fries, N. Mahnke and E. Stein, Nucl. Phys. B 589 (2000) 381, [Erratum-ibid. B 607 (2001) 433].

[6] V. M. Braun, A. Lenz, N. Mahnke and E. Stein, Phys. Rev. D 65 (2002) 074011.

[7] A. Lenz, M. Wittmann and E. Stein, Phys. Lett. B 581 (2004) 199.

[8] V. M. Braun, A. Lenz and M. Wittmann, Phys. Rev. D 73 (2006) 094019.

[9] A. Lenz, M. Gockeler, Th. Kaltenbrunner and N. Warkentin, Phys. Rev. D 79 (2009) 093007

[10] B. L. Ioffe, Nucl. Phys. B 188 (1981) 317 [Erratum-ibid. B 191 (1981) 591].

[11] V. M. Belyaev, V. M. Braun, A. Khodjamirian and R. Ruckl, Phys. Rev. D 51 (1995) 6177.

[12] M. Q. Huang and D. W. Wang, Phys. Rev. D 69 (2004) 094003.

[13] Y. M. Wang, Y. Li and C. D. Lü, Eur. Phys. J. C 59 (2009) 861. 
[14] Y. M. Wang, Y. L. Shen and C. D. Lü, Phys. Rev. D 80 (2009) 074012.

[15] K. Azizi, M. Bayar, Y. Sarac and H. Sundu, Phys. Rev. D 80 (2009) 096007.

[16] T. M. Aliev, K. Azizi and M. Savci, Phys. Lett. B 696 (2011) 220.

[17] Y. B. Dai, C. S. Huang, M. Q. Huang and C. Liu, Phys. Lett. B 387 (1996) 379.

[18] C. S. Huang, C. F. Qiao and H. G. Yan, Phys. Lett. B 437 (1998) 403.

[19] F. S. Navarra and M. Nielsen, Phys. Lett. B 443 (1998) 285.

[20] R. S. Marques de Carvalho, F. S. Navarra, M. Nielsen, E. Ferreira and H. G. Dosch, Phys. Rev. D 60 (1999) 034009.

[21] K. Nakamura et al. [Particle Data Group], J. Phys. G 37 (2010) 075021.

[22] E. Bagan, M. Chabab, H. G. Dosch and S. Narison, Phys. Lett. B 301 (1993) 243.

[23] D. Jido, N. Kodama and M. Oka, Phys. Rev. D 54 (1996) 4532.

[24] E. V. Shuryak, Nucl. Phys. B 198 (1982) 83.

[25] Y. Chung, H. G. Dosch, M. Kremer and D. Schall, Nucl. Phys. B 197 (1982) 55.

[26] V. M. Braun, A. N. Manashov and J. Rohrwild, Nucl. Phys. B 807 (2009) 89.

[27] V. M. Braun, T. Lautenschlager, A. N. Manashov and B. Pirnay, Phys. Rev. D83 (2011) 094023.

[28] E. Bagan, M. Chabab, H. G. Dosch and S. Narison, Phys. Lett. B 287 (1992) 176.

[29] E. Bagan, M. Chabab, H. G. Dosch and S. Narison, Phys. Lett. B 278 (1992) 367.

[30] A. Khodjamirian, Ch. Klein, Th. Mannel and Y.-M. Wang, paper in preparation.

[31] Z.-G. Wang, Eur. Phys. J. C 68 (2010) 479.

[32] K. G. Chetyrkin, J. H. Kühn, A. Maier, P. Maierhofer, P. Marquard, M. Steinhauser and C. Sturm, Phys. Rev. D 80 (2009) 074010.

[33] G. Duplancic, A. Khodjamirian, Th. Mannel, B. Melic and N. Offen, JHEP 0804 (2008) 014.

[34] A. Khodjamirian, Ch. Klein, Th. Mannel and N. Offen, Phys. Rev. D 80 (2009) 114005 .

[35] P. Ball, V. M. Braun and E. Gardi, Phys. Lett. B 665 (2008) 197.

[36] V. L. Chernyak and I. R. Zhitnitsky, Nucl. Phys. B 246 (1984) 52.

[37] C. Bourrely, I. Caprini and L. Lellouch, Phys. Rev. D 79 (2009) 013008. 
[38] A. Khodjamirian, Th. Mannel, N. Offen and Y.-M. Wang, Phys. Rev. D 83 (2011) 094031.

[39] C. D. Lü, Y. M. Wang, H. Zou, A. Ali and G. Kramer, Phys. Rev. D 80 (2009) 034011.

[40] T. Aaltonen et al. [CDF Collaboration], Phys. Rev. Lett. 103 (2009) 031801. 